\title{
THE MOLECULAR FORMS OF BMP15 IN A RANGE OF MAMMALIAN SPECIES
}

\section{Gene William Swinerd}

A thesis submitted to the Victoria University of Wellington in fulfilment of the requirements for the degree of Masters in Biomedical Science

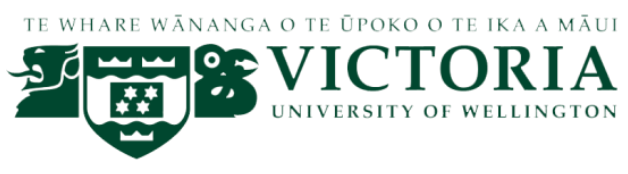

Victoria University of Wellington

Te Whare Wānanga o te Ūpoko o te Ika a Māui 
This thesis is dedicated to my family and friends 


\section{Abstract}

BMP15 is an oocyte-secreted growth factor that is critical for ovarian follicular development and fertility in mammals. To improve our understanding of the speciesspecific effects of BMP15 on fertility, the aim of this study was to elucidate the molecular forms of BMP15 in mammals with high (rat and pigs) and low (sheep and deer) litter sizes. Western blotting experiments were undertaken using a monoclonal antibody directed against a semi-conserved area on the mature region of E.coli-expressed ovine BMP15.

Within the oocyte lysate of all species tested, the predominant form was promature BMP15, although mature BMP15 was also present. Within oocyte-conditioned media of sheep, pigs and deer, the promature:mature BMP15 ratio dropped significantly from what was seen inside the oocyte, with promature BMP15 only slightly predominant in deer, and relatively equal amounts of each protein were present in pigs and sheep. No BMP15 was detected in the oocyte-conditioned media of rats. Cross-linking studies did not provide any evidence of BMP15/BMP15 homodimers or BMP15/GDF9 heterodimers in any species tested.

Intra-oocyte levels of both promature and mature BMP15, from highest to lowest, were detected in deer, followed by sheep and pigs (similar), and then rats (significantly lower). In the oocyte-conditioned media, with the exception of the rat where BMP15 was absent, there was a similar pattern of promature BMP15 levels observed between species, whereas mature BMP15 levels showed no inter-species variation.

In summary, the molecular forms and relative amounts of BMP15 protein differ across species. Generally, high BMP15 levels were associated with low litter size; however the pig is an exception. Furthermore, the molecular forms observed in this study differed from those reported in studies using recombinant BMP15 suggesting that recombinantlyexpressed protein may not provide a fair representation of native BMP15. 


\section{Acknowledgements}

I would like to first of all thank my supervisor, Dr Janet Pitman, for agreeing to let me work on this project and for providing a huge amount of support above any beyond what was expected or required. The patience and encouragement from Janet was a massive help during the most difficult stages of the project, and was particularly helpful with alleviating the stress which came during the writing stage. I would also like to thank Prof Ken McNatty, Derek Heath (soon to be Dr Derek Heath), and Adrian Bibby for helping troubleshoot all the problems which arose during experiments. I am also grateful to the rest of the reproductive team at Victoria University of Wellington who made me feel like a welcome part of the group, helped with any questions I had, and also assisted me in collecting oocytes on occasion.

Thank you to the Department of Psychology at Victoria University of Wellington; the Taylor Preston meat processing plant in Ngauranga, Wellington; the Venison Packers Feilding Ltd meat processing plant in Fielding; and Cabernet Foods, Kintyre Meats Ltd in Gladstone for providing access to the ovaries required for this research, and for being friendly and helpful despite having their own busy schedules.

At this point I wish to acknowledge funding support from the Royal Society of NZ and the Marsden fund (Contract 13-VUW-153).

Lastly, I would like to thank my family, friends, and girlfriend Patricia for providing support while I was stressed, and for putting up with me often being busy during the later stages of the thesis. 


\section{Table of Contents}

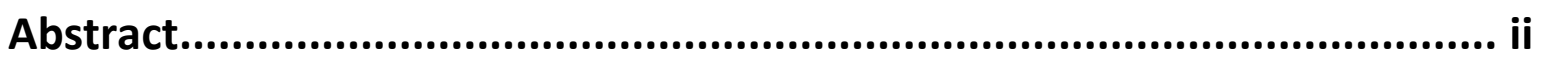

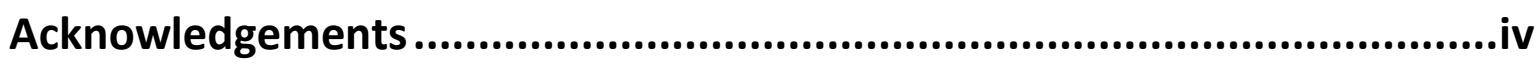

Table of Contents .......................................................................................

List of Figures ............................................................................................... viii

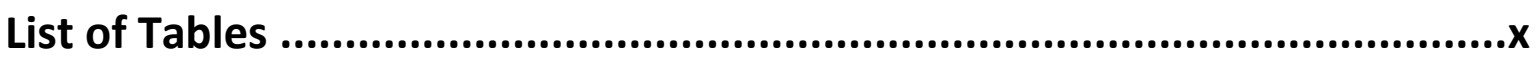

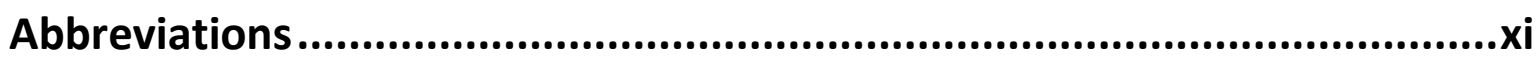

Chapter 1: Introduction ........................................................................... 1

1.1 The Transforming Growth Factor-Beta (TGF- $\beta$ ) Superfamily of Proteins ............................ 1

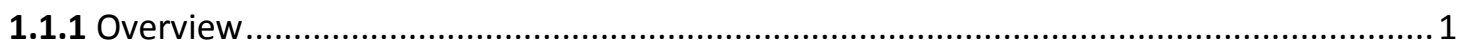

1.1.2 General Structure of TGF- $\beta$ Superfamily Proteins ................................................. 2

1.1.3 General Receptor Structure and Signalling Mechanisms of TGF- $\beta$ Superfamily Proteins. 3

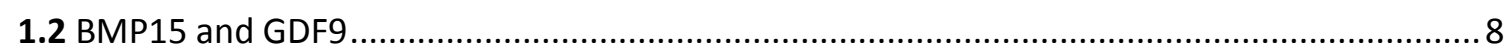

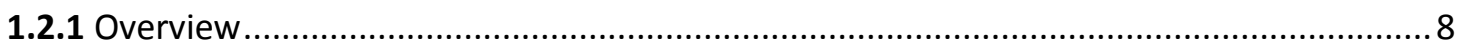

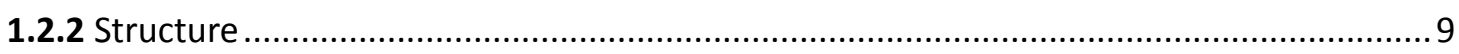

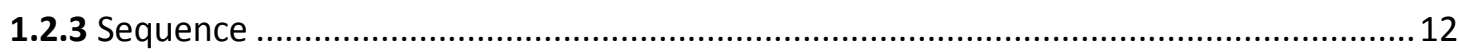

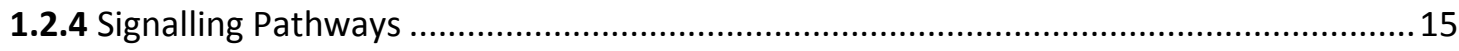

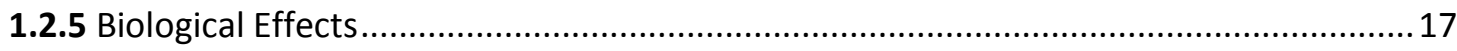

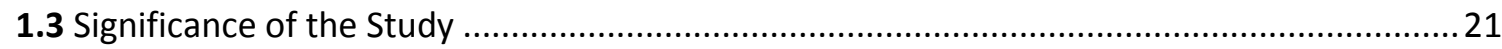

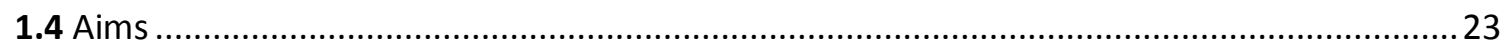

Chapter 2: Materials and General Methodology.................................... 25

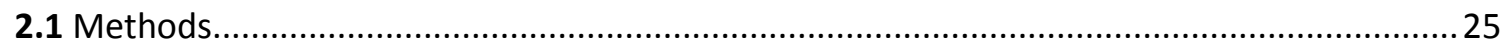

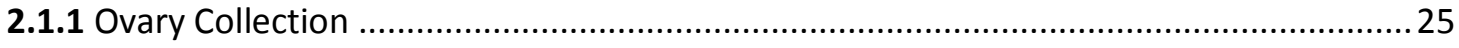

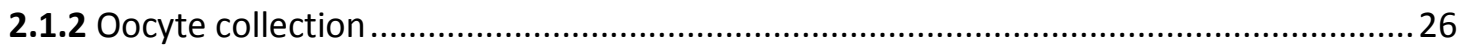

2.1.3 Western blotting.................................................................................... 28

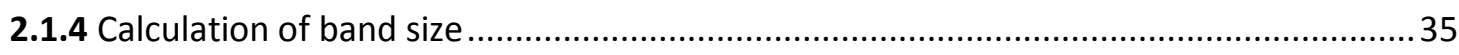

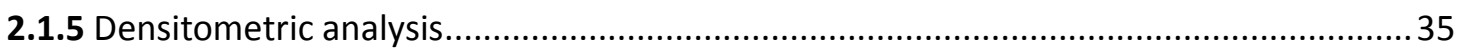

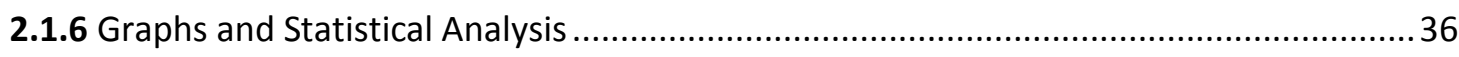

Chapter 3: Results ................................................................................ 38 
3.1 The monoclonal antibody Mab61B has a significantly reduced ability to detect promature BMP15, and a moderately reduced ability to detect mature BMP15 on a Western blot, compared to Mab61A.

3.2 The monoclonal antibodies Mab61B and Mab61A have different non-specific binding targets, and different levels of specificity for their BMP15 target

3.3 High affinity non-specific bands in close proximity to specific target bands on Western blots may partially block the primary antibody from binding its specific target.

3.4 The optimal numbers of oocytes for detection of intra-oocyte molecular forms of BMP15 in rats, sheep, pigs and deer are 200,100, 80 and 100, respectively . .46

3.5 Estimation of the concentration of mature BMP15 in the recombinant HEK-293 produced pig BMP15 conditioned media......

3.6 The molecular forms of BMP15 in, and secreted from, oocytes of rats (litter size 7-16) ......50

3.7 The molecular forms of BMP15 in, and secreted from, oocytes of sheep (litter size 1-3) .....54

3.8 The molecular forms of BMP15 in, and secreted from, oocytes of pigs (litter size 14-22) ....57

3.9 The molecular forms of BMP15 in, and secreted from, oocytes of red deer (litter size=1) ...60

3.10 The alignment of intra-oocyte and oocyte-secreted molecular forms of BMP15 with those of GDF9 in rats, sheep, pigs and deer.

3.11 Models for the quantification and normalisation of the BMP15 band densities across blots

3.12 Comparison between the levels of intra-oocyte and oocyte secreted promature and mature forms of BMP15 in rats, sheep, pigs and deer normalised using $\beta$-actin ......

3.13 Comparisons between the levels of intra-oocyte and oocyte-secreted promature and mature forms of BMP15 in rats, sheep, pigs and deer with the levels expressed relative to the number of oocytes per sample, and normalised using the mature BMP15 band in the positive control.

3.14 Differences in the ratio of promature:mature BMP15 both within the oocyte and secreted by the oocyte in rats, sheep, pigs and deer......

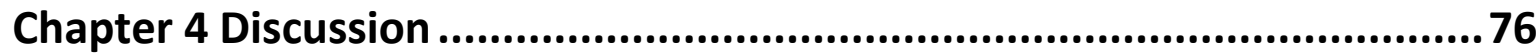

4.1 Comparisons between two different monoclonal antibodies which were generated to target different areas within the mature BMP15 protein . .76

4.2 The intra-oocyte and oocyte-secreted molecular forms of BMP15 across a range of mammalian species with different litter sizes (rats, sheep, deer and pigs)

4.3 The relative amounts of promature and mature BMP15 across a range of mammalian species with different litter sizes (rats, sheep, deer and pigs)

4.4 Study Conclusions

4.5 Future Directions . .90 


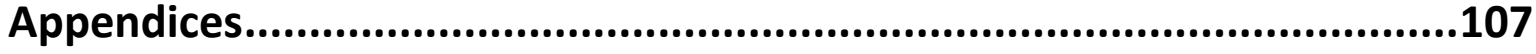

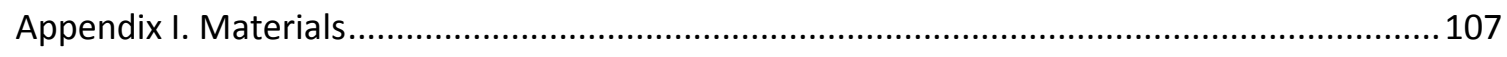

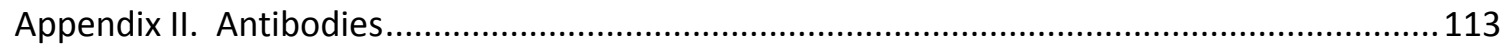

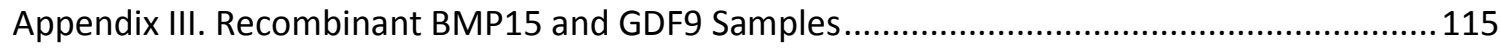

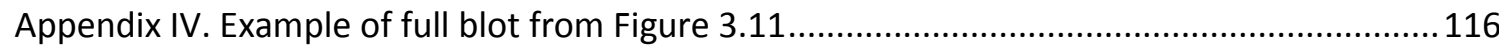




\section{List of Figures}

Figure 1.1: The structure of TGF- $\beta$ superfamily proteins and their receptors ...........6

Figure 1.2: The TGF- $\beta$ Smad signalling pathway in target cells ......................... 7

Figure 1.3: The BMP15 sequence in rats, pigs, sheep and deer ..................... 14

Figure 3.1: Mab61A showed a low affinity for recombinant promature BMP15 compared to Mab61A, even following an optimised Western blot protocol........... 40

Figure 3.2: Mab61B shows no affinity for the promature BMP15 protein in oocyte lysates, as compared to Mab61A................................................... 41

Figure 3.3: Preabsorption of Mab61B and Mab61A antibodies differ with antigen, and exhibit divergent non-specific binding on Western blots 43

Figure 3.4: Mab61B can bind promature BMP15 on Western blots when it is separated from non-target proteins of a similar size....

Figure 3.5: Titrations of rat, sheep, deer and pig lysed oocyte numbers for the detection of BMP15 using Mab61A

Figure 3.6: Estimation of the mature BMP15 concentration in the recombinant HEK-293 produced pig BMP15 conditioned media.

Figure 3.7: The molecular forms of BMP15 present in, and secreted from, rat oocytes 53

Figure 3.8: The molecular forms of BMP15 present in, and secreted from sheep oocytes.

Figure 3.9: The molecular forms of BMP15 present in, and secreted from pig oocytes 59

Figure 3.10: The molecular forms of BMP15 present in, and secreted from deer oocytes 62

Figure 3.11: Comparisons between the intra-oocyte and oocyte secreted molecular forms of BMP15 and GDF9 detected using Mab61A and Mab37A, respectively. 65

Figure 3.12: Tests performed to investigate reliability of the quantification and normalisation of the BMP15 band densities across blots 68 
Figure 3.13: Levels of intra-oocyte and oocyte-secreted promature and mature BMP15 detected under reducing conditions in each species relative to the $\beta$-actin levels detected for each species

Figure 3.14: Levels of intra-oocyte and oocyte-secreted promature and mature BMP15 detected under reducing conditions in each species presented relative to the positive control mature BMP15 band, and adjusted for the number of oocytes used per sample.

Figure 3.15: Differences in the promature to mature ratio in lysates and conditioned media from in rat, sheep, deer and pig oocytes under reducing conditions

Appendix II Figure 1: The mature BMP15 target sequences for Mab61A and Mab61B 115

Appendix IV Figure 1: Full blots representing sheep BMP15 and GDF9 under reducing conditions 


\section{List of Tables}

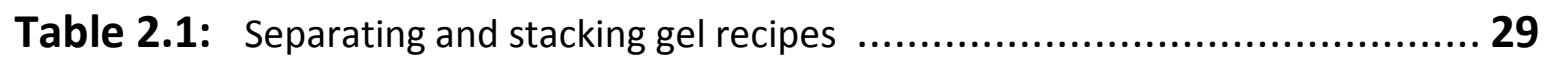

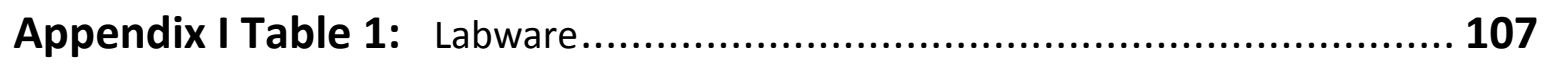

Appendix I Table 2: Reagents and Materials ............................................. 108

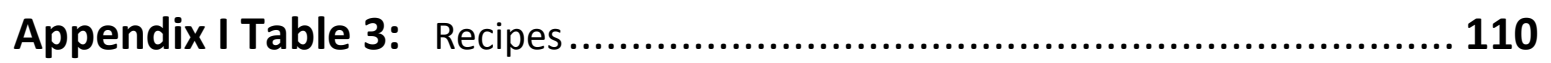

Appendix I Table 4: Antibodies Summary ............................................... 112 


\section{Abbreviations}

\begin{tabular}{|c|c|}
\hline ActRII/B & Activin receptor type II or type IIB \\
\hline ALK & Activin receptor-like kinase \\
\hline AMHR-II & Anti-Müllerian hormone receptor type II \\
\hline ANOVA & Analysis of variance \\
\hline Arg & Arginine \\
\hline BMP & Bone morphogenetic protein \\
\hline BMPRII & Bone morphogenetic protein receptor type 2 \\
\hline BS3 & Bissulfosuccinimidyl suberate \\
\hline CC & Cumulus cell \\
\hline $\mathrm{COC}$ & Cumulus-oocyte-complex \\
\hline DMEM & Dulbecco's Modified Eagle Medium \\
\hline DO & Denuded oocytes \\
\hline DTT & Dithiothreitol \\
\hline ERK1/2 & Extracellular signal-regulated kinases $1 / 2$ \\
\hline $\mathrm{FSH}$ & Follicle stimulating hormone \\
\hline GC & Granulosa cell \\
\hline GDF & Growth differentiation factor \\
\hline Gly & Glycine \\
\hline HEK-293 cells & Human Embryonic Kidney 293T cells \\
\hline IVF & In vitro fertilisation \\
\hline IVM & In vitro maturation \\
\hline KLH & Keyhole limpet hemocyan \\
\hline LH & Luteinising hormone \\
\hline MAPK & Mitogen-activated protein kinase \\
\hline OSF & Oocyte-secreted factor \\
\hline
\end{tabular}


PBS

pED-IRES

SDS

SDS-PAGE

Smad

TF

TGF- $\beta$

$T \beta R-I I$
Phosphate buffered saline

Elongation factor- internal ribosomal entry site plasmid

Sodium dodecyl sulfate

Sodium dodecyl sulfate polyacrylamide gel electrophoresis

Similar to mothers against decapentaplegic

Transcription factor

Transforming growth factor $\beta$

TGF- $\beta$ type II receptor 


\section{Chapter 1: Introduction}

\subsection{The Transforming Growth Factor-Beta (TGF- $\beta$ ) Superfamily of Proteins}

\subsubsection{Overview}

The TGF- $\beta$ superfamily of proteins is a large group of structurally-related proteins that regulate a range of cellular processes throughout the body in vertebrates, including development, growth, differentiation, adhesion, positioning, movement and reproductive function (Chang et al., 2002; Lin et al., 2006; Massagué, 2012). The superfamily can be divided into four subfamilies based on the genetic structure and origin of each protein: namely the activins/inhibins, the TGF- $\beta$ subfamily, the bone morphogenetic proteins (BMPs) and growth differentiation factors (GDFs) and finally, a group consisting of distant and diverse members which don't fall into any of the first three subfamilies (Mueller and Nickel, 2012). The activin/inhibin subfamily have roles including the regulation of folliclestimulating hormone (FSH) expression and the menstrual cycle, while the TGF $\beta$ s subfamily members regulate tissue homeostasis, and have roles in cell proliferation, cell differentiation, and embryonic development (Macias et al., 2015; McCartney-Francis and Wahl, 1994; Mueller and Nickel, 2012). The BMPs and GDFs form the largest subfamily with more than 15 members, and regulate limb, embryonic, skeletal and other organ development (Mueller and Nickel, 2012). The members of the fourth and final subfamily do not have a defined relationship other than they all fall outside of the other three subfamilies whilst still meeting the classification criteria for TGF- $\beta$ proteins, and includes both BMP15 and GDF9 (Mueller and Nickel, 2012).

Many important growth factors involved in mammalian folliculogenesis are members of the TGF- $\beta$ superfamily, and are expressed in a coordinated manner to regulate the various stages of development (Knight and Glister, 2006; Paradis et al., 2009). These include BMP4 and BMP7 by theca cells, inhibins and activins by granulosa cells (GCs), and BMP15 and GDF9 by oocytes (Shimasaki et al., 2004a). It is important to understand the shared characteristics of the TGF- $\beta$ superfamily considering that so many members play a role in 
mammalian fertility, and that these members share structural characteristics and a common ancestry.

\subsubsection{General Structure of TGF- $\beta$ Superfamily Proteins}

There are over 35 members in the TGF- $\beta$ superfamily in vertebrates, all of which are classified based on their similar structure (Knight and Glister, 2006). Members are synthesised as a preproprotein consisting of a signal peptide (the pre-region), a large precursor segment with a chaperone function (the proregion), and a mature domain at the carboxy-terminal (the mature region) (see Figure 1.1 A) (Chang et al., 2002; Shimasaki et al., 2004a). It was previously suggested that the proregion was required for correct folding and dimerization of the mature TGF- $\beta$ ligands, however recombinant forms of the mature segment alone have been shown to form dimers and fold correctly in vitro which indicates that the proregion is not always required for these purposes (Fairlie et al., 2001; Harrison et al., 2011; Mueller and Nickel, 2012; Ruppert et al., 1996). The mature segment is preceded by a conserved RXXR motif that is recognised by proprotein convertases such as furin (Juengel and McNatty, 2005; Seidah and Chrétien, 1999). Under the current model of protein processing, the pre-region is removed following translocation into the rough endoplasmic reticulum (RER) and then the proprotein forms a dimer, which in turn is enzymatically cleaved at the RXXR region leaving a mature dimer (Juengel and McNatty, 2005; McPherron and Lee, 1993). Post-translational processing in the ER and Golgi apparatus, such as $\mathrm{N}$ - and $\mathrm{O}$-glycosylation, may be essential for the bioactivity of the proteins (Brunner et al., 1992; Hang et al., 2014; Hashimoto et al., 2005; Miyazono and Heldin, 1989; Saito et al., 2008).

In many cases, even though the proregion has been enzymatically cleaved, it may remain associated with the mature region through non-covalent interactions and continue to regulate its activity. In such cases, the mature protein bioactivity is often delayed by the presence of the associated proregion until it is actively removed (see Figure $1.1 \mathrm{~B}$ ) (Hauburger et al., 2009; Mclntosh et al., 2008; McMahon et al., 1996; Mueller and Nickel, 2012). The proregion has more sequence variability between TGF- $\beta$ members than the 
mature region, with some members forming disulphide bonds between two proregions while others do not (see Figure 1.1 B) (Mueller and Nickel, 2012; Shi et al., 2011). These differences may affect the strength with which the proregions remain associated with their corresponding mature segments (Mueller and Nickel, 2012).

In general, members of the TGF- $\beta$ superfamily have seven conserved cysteine residues within the mature segment. Three pairs of these cysteine residues form intramolecular disulphide bonds, causing a 3-dimensional cysteine knot structure to form. In addition, one of the residues in each mature protein forms a covalent intermolecular disulphide bond with another mature protein to form a mature dimer (see Figure 1.1 B) (Lin et al., 2006; McPherron and Lee, 1993; Shimasaki et al., 2004a). These mature dimers are usually homodimers, that is a dimer of two matching mature protein subunits, however heterodimers between closely related proteins within the TGF- $\beta$ superfamily have been reported (Guo and Wu, 2012; Shimasaki et al., 2004a). These heterodimers tend to be characterised by unique and generally-enhanced functions, compared to their homodimer counterparts (Guo and Wu, 2012). A small subset of the TGF- $\beta$ superfamily, namely GDF3, GDF9, BMP15, Lefty1 and Lefty2, lack the seventh cysteine residue which means they can't form the disulphide bridge between the two subunits (Mueller and Nickel, 2012; Shimasaki et al., 2004a). Instead, they may form as non-covalent homodimers and heterodimers (Lin et al., 2006; McIntosh et al., 2008; Mottershead et al., 2015).

\subsubsection{General Receptor Structure and Signalling Mechanisms of TGF- $\beta$ Superfamily Proteins}

Members of the TGF- $\beta$ superfamily are cytokines which bind to receptor serine kinases on the surface of cells. There are hundreds of serine/threonine kinases, however only twelve are cell surface-bound receptors, all of which are receptors for TGF- $\beta$ superfamily members (Macias et al., 2015). These receptors generally phosphorylate Smad [from Caenorhabditis elegans SMA (small) and Drosophila mothers against decapenthaplegic] related proteins, allowing them to form complexes that act as transcription factors to regulate gene 
expression (Macias et al., 2015). The target genes depends upon a range of factors such as the receptor complex to which the TGF- $\beta$ member binds, the Smad proteins involved, the Smad transcriptional co-factors employed, the epigenetic status, as well as other DNA binding partners and mediators (see Figure 1.2) (Massagué, 2012; Massagué et al., 2005). These factors are highly contextual and vary depending on the type and developmental stage of the target cell, giving the TGF- $\beta$ family members a vastly complex arsenal of possible cellular effects (Macias et al., 2015; Massagué, 2012).

The twelve serine/threonine kinase surface receptors fall into two categories, namely the type I and type II receptors. Both receptor types have an intracellular domain with serine/threonine kinase activity, a single pass transmembrane domain, and an extracellular ligand binding domain with a three-finger toxin fold (de Caestecker, 2004). The two categories are differentiated by the presence of a region rich in glycine/serine (GS-box) on the intracellular side of type I receptors, and its absence in type 2 receptors (see Figure 1.1 C) (Mueller and Nickel, 2012). In general, the binding of the dimeric ligand causes two type I and two type II receptors to assemble in a complex (Massagué, 2012). Most TGF- $\beta$ members appear to bind to a type II receptor first, and then subsequently recruit a type I receptor, however many BMPs (not including BMP15) appear to have a greater affinity for type I receptors than type II receptors and may prefer to bind a pre-formed receptor complex made of type I and type II receptors (Mueller and Nickel, 2012; Rosenzweig et al., 1995; ten Dijke et al., 2003). Once in the complex, the type II receptors phosphorylate and activate the type I receptors, and these then phosphorylate and activate the Smad signalling proteins and/or other signal delivery factors (Massagué, 2012; Wrana et al., 1994).

There are seven type I (ALK1-7) and five type II (ActR-II, ActR-IIB, TßR-II, BMPR-II and AMHRII) cell-surface serine/threonine receptors, and these are able to form unique combinations with specificities for the various TGF- $\beta$ superfamily ligands (Mueller and Nickel, 2012). The expression pattern of these receptors may affect how different cells respond to the same TGF- $\beta$ ligands (Massagué, 2012). The level of signal a cell receives from stimulation by a TGF- $\beta$ superfamily ligand can be altered by several factors such as the concentration of the TGF- $\beta$ ligand present, the ligand subtype and its affinity for the receptor, the presence of ligand binding protein or traps which can capture the TGF- $\beta$ ligands and prevent binding, 
and also the presence of mediators which regulate the release of the ligand from the traps (Cheifetz et al., 1987; Massagué, 2012; Zakin and De Robertis, 2010). Some TGF- $\beta$ ligands may bind the receptors of other TGF- $\beta$ members without causing any downstream signal activation, and thus inhibit their activity (Massagué, 2012). An example of this are the inhibins, which can block activin from binding its receptors (Zhu et al., 2012).

The first Smads to undergo phosphorylation by the type I receptor are receptor-regulated Smad molecules (rSmads). The rSmads which are phosphorylated by the type I receptor are either Smad1, 5 and 8, or Smad2 and 3 depending on the ligand and the type I receptor involved (Massagué, 2012). The rSmads then form a complex with Smad4 (commonmediator Smad, or cSmad) which then translocates into the nucleus and regulate gene expression (Mueller and Nickel, 2012). Smad4 can be inhibited by inhibitory Smads (iSmads; Smad6 and 7) that can act by forming an inactive complex with Smad4 or by binding the activated type I receptor in place of rSmads and thus prevent their activation (see Figure 1.2) (Hariharan and Pillai, 2008; Mueller and Nickel, 2012). Other Smad independent pathways exist for some TGF- $\beta$ superfamily members, such as the mitogen activated protein kinase (MAPK) pathway (Moore et al., 2003; Moustakas and Heldin, 2005; Reader et al., 2011; Sano et al., 1999). 
TGF- $\beta$ Superfamily General Structure

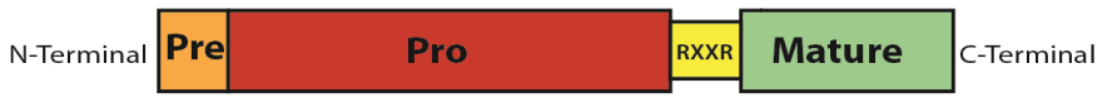

Covalent Promature

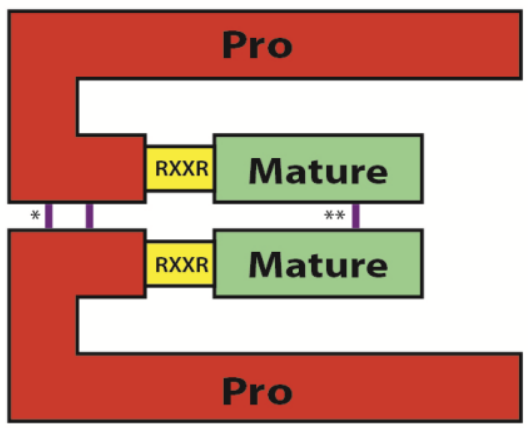

C

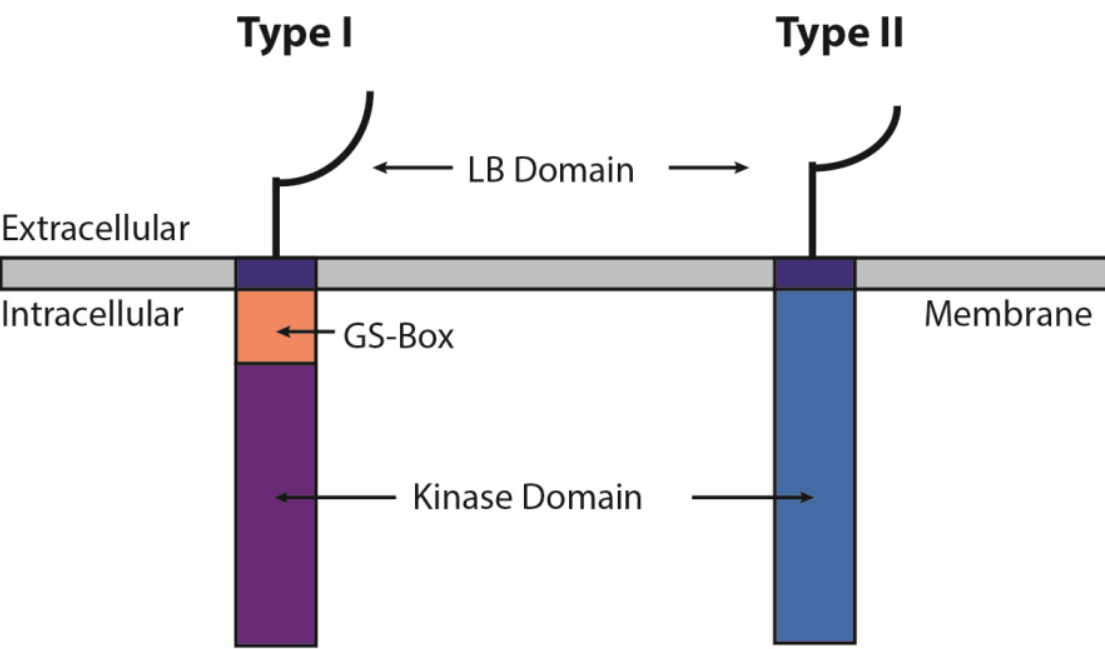

Non-covalent Promature

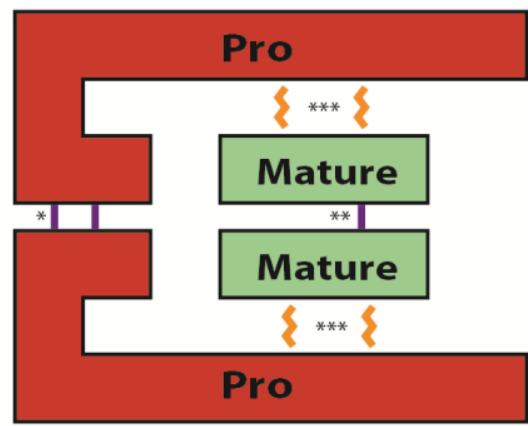

Mature

Mature

Mature

Figure 1.1. The structure of TGF- $\beta$ superfamily proteins and their receptors. (A) General structure of TGF- $\beta$ superfamily proteins. TGF- $\beta$ superfamily members are synthesised as prepromature proteins with an $\mathrm{N}$-terminal signal peptide (the preregion) which is removed upon translocation into the RER, a large proregion with chaperone function, and mature peptide at the C-terminal. The mature peptide is preceded by a RXXR residue which is targeted by proteases such as furin. (B) The protein forms a dimeric complex. Represented by a single asterisk $\left({ }^{*}\right)$ are the intermolecular disulphide binds found between the prodomains of some members of the TGF- $\beta$ superfamily such as those within the TGF- $\beta$ subfamily. The double asterisk $\left({ }^{* *}\right)$ represents the disulphide bond between the mature protein dimer in all TGF- $\beta$ superfamily members except for BMP15, GDF9, GDF3, Lefty1 and Lefty2. The protein may exist as either a promature dimer, a mature dimer with non-covalently associated proregions or a mature dimer which fully disassociated from the proregions. The triple asterisk $\left({ }^{* * *}\right)$ represents non-covalent association. (C) The structure of the type I and type II TGF- $\beta$ receptors. The ligand binding domain (LB domain) lies on the extracellular side of the cell membrane and is connected to the intracellular kinase domain by a single pass transmembrane region. The type I receptor has a glysine/serine rich region (GS Box) on the intracellular side of the membrane which plays a role in the phosphorylation of downstream Smad proteins. The figures were created using Adobe Illustrator with descriptions in Massagué, 2012 and Mueller \& Nickel, 2012 used as a guide. 


\section{TGF $\beta$}

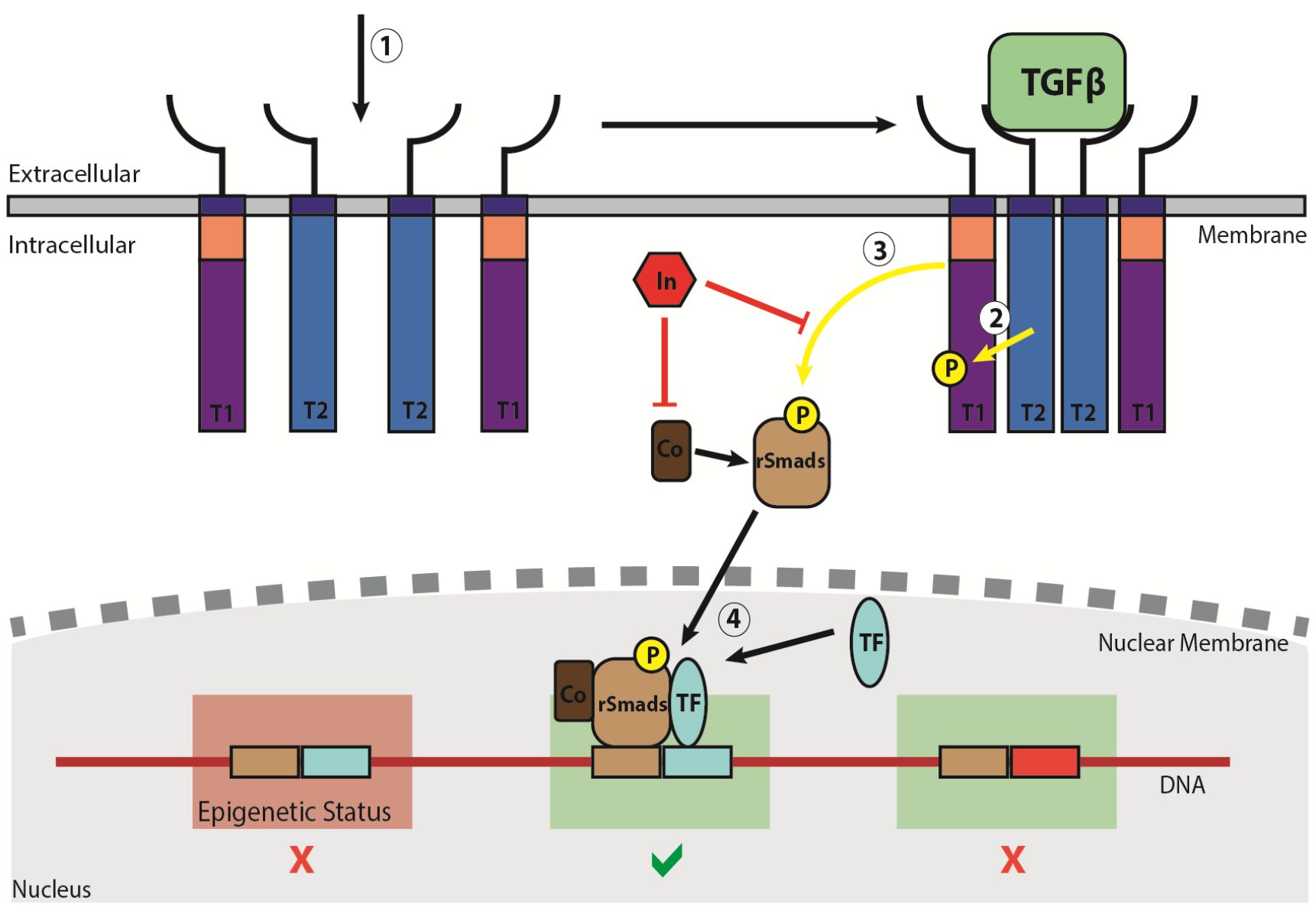

Figure 1.2. The TGF- $\beta$ Smad signalling pathway in target cells. (1) TGF- $\beta$ binds to a complex of type I (T1) and type II (T2) receptors on the surface of the target cell. Generally, the TGF- $\beta$ dimeric ligand will bind a pair of type II receptors which then recruit a pair of type I receptors. (2) The constitutively active type II receptors then phosphorylate the type I receptors in the complex. (3) The phosphorylated type I receptors then bind and phosphorylate rSmads. At this stage, a range of signal mediators such as the common-mediator Smad4 (Co) bind the rSmads. Inhibitory Smads such as Smad 6 and 7 (In) can inhibit the signalling through either binding Smad4 to form an inactive complex, or by binding the phosphorylated type I receptor in place of the rSmads, preventing their phosphorylation. (4) Once the phosphorylated rSmads have successfully formed a complex with the required signal mediators they translocate into the nucleus and interact with a range of other cell lineage specific transcription factors (TF). The range of transcription factors and other signal mediators in the complex determines which genes are targeted and whether they are activated or repressed. The epigenetic status of the target gene determines whether it is accessible to the rSmad complex (shaded in red and green, where red represents an inaccessible gene while green is accessible). The components of the transcription complex must match the correct enhancer elements preceding the target gene for transcription to occur. Successful transcription is marked by a green tick. The figures were created using Adobe Illustrator with descriptions and images in Massagué, 2012 used as a guide. 


\subsection{BMP15 and GDF9}

\subsubsection{Overview}

In the past, it was believed that folliculogenesis was controlled solely by endocrine factors such as protein and steroidal hormones. However, in recent years the importance of intrafollicular paracrine signals has been established by an ever increasing abundance of studies (Paulini and Melo, 2011). These studies have revealed the essential role of paracrine communication via a range of oocyte secreted factors (OSFs), between a developing oocyte and the surrounding cumulus and granulosa cells of a growing follicle (Gilchrist et al., 2008; Otsuka et al., 2011). Two OSFs that have been the subject of a significant amount of research are BMP15 (previously named GDF9B), which is the protein of interest in this study, and GDF9. It is important to include GDF9 when considering BMP15 as the two proteins have closely-related and largely intertwining roles. Their roles extend from the very early stages of follicular development, as early as the primordial follicle stage, and continue right through to ovulation, and thus form a vital component of mammalian fertility (Sun et al., 2010). Their roles appear to be species-specific, and include the regulation of the intra-follicular somatic cells. In particular, these two OSFs promote proliferation of, and steroidogenesis in, granulosa cells, inhibit apoptosis and luteinisation of these cells and regulate the differentiation of a subset of granulosa cells into specialised cumulus cells (Dragovic et al., 2007; Gilchrist et al., 2008; Knight and Glister, 2006; Li et al., 2000; McNatty et al., 2005a; Otsuka and Shimasaki, 2002).

GDF9 was the first of the two proteins to be discovered, with its discovery in the mouse ovary in 1993 (McPherron and Lee, 1993). BMP15 was discovered a few years later in 1998 (Dube et al., 1998). They are the most homologous of all the OSFs, and are more closely related to each other ( $53 \%$ homology in mice) than they are to any other member of the TGF- $\beta$ superfamily of proteins (Laitinen et al., 1998). The current evidence is that in rodents, sheep, pigs, deer and a range of other mammals, both Bmp15 and Gdf9 mRNA are only expressed by the oocyte within the follicle (Aaltonen et al., 1999; Bodensteiner et al., 2000; Juengel and McNatty, 2005; Laitinen et al., 1998). However, despite being oocyte-specific within the ovary, BMP15 and GDF9 has been detected in extra-ovarian tissues such the 
testis and pituitary gland in some species (Fitzpatrick et al., 1998; Otsuka and Shimasaki, 2002; Wang et al., 2009).

The relative expression levels of Gdf9 and Bmp15 mRNA has been shown to be highly variable between different mammalian species. However, relatively equal- or low-ratios of GDF9:BMP15 was generally associated with low or mono-ovulatory species such as sheep, cows and red deer, while a high GDF9 to BMP15 ratio was found in poly-ovulatory species such as rats (Crawford and McNatty, 2012). This trend aligns well with a number of studies where it was found GDF9, but not BMP15, was essential for follicular development in polyovulatory species such as mice, while both proteins were required for normal folliculogenesis in species with a low ovulation rate, such as the sheep (Galloway et al., 2000; Hanrahan et al., 2004; Yan et al., 2001). There were a few notable outliers to the trend such as the pig, which had a low GDF9:BMP15 ratio despite producing large litters (i.e. high ovulation rate species) (Crawford and McNatty, 2012).

\subsubsection{Structure}

The primary structure of both proteins follows the same basic structure found in all TGF- $\beta$ family members as described in Section 1.1.2. They are produced as preproproteins, with an $\mathrm{N}$-terminal signal peptide (pre-region) which is removed upon translocation into the RER, a proregion, and then mature region at the C-terminal (Chang et al., 2002). The proregion is cleaved from the mature region by proprotein convertases at a four amino acid motif (RXXR) directly preceding the $\mathrm{N}$-terminal of the mature peptide (Juengel and McNatty, 2005). BMP15 and GDF9 belong to a small group within the TGF- $\beta$ superfamily which lack the fourth of the seven conserved cysteines within the sequence of their mature peptide, and as such are unable to form a covalent bond during dimer formation (Mueller and Nickel, 2012; Shimasaki et al., 2004a). The lack of a covalent bond within the dimers is presumed to allow for a more flexible array of possible interactions between monomer units than is traditionally seen with other TGF- $\beta$ family members (Chang et al., 2002). Modelling of the BMP15 and GDF9 heterodimer interface reveals similarities to the homodimer interface for each protein, and demonstrates that a stable non-covalent 
heterodimer complex is possible (Mottershead et al., 2015). Numerous in vitro studies have observed homodimers, heterodimers and even multimers between the two recombinant proteins (Liao et al., 2003; Lin et al., 2006; Mclntosh et al., 2008; Mottershead et al., 2015; Peng et al., 2013a; Shimasaki et al., 2004a) however to date, dimers between native oocyteproduced proteins in solution are yet to be identified.

The activity of BMP15 and GDF9 varies greatly between species, with differences in the molecular forms of each protein likely to play a large role in this. Granulosa cell expansion assays with recombinant human and mouse BMP15 and GDF9 heterodimers revealed a 3000 fold increase in activity with human heterodimers compared to their BMP15 homodimer counterparts, whilst the human GDF9 homodimers had little to no activity (Peng et al., 2013a). Conversely, there was a 10 to 30 fold increase in activity with mouse heterodimers compared to their GDF9 homodimer counterpart, while mouse BMP15 homodimers were essentially inactive (Peng et al., 2013a). This highlights the difference in activity between homodimers and heterodimers of each protein, and also the species specific nature of the activity of each protein form (Peng et al., 2013a).

Like many other members of the TGF- $\beta$ family, the proregion has been shown to remain non-covalently associated with both GDF9 and BMP15 after cleavage at its protease processing site, and it may have a role in regulating the bioactivity of each protein along with their cooperative interactions (McIntosh et al., 2012, 2008). It has been demonstrated that recombinant mouse BMP15 is secreted in a cleaved and uncleaved form, while mouse GDF9 is only secreted in a cleaved form. The cleaved proregion tends to remain noncovalently associated with the mature region of each protein, however the proregion only remains weakly associated with mouse GDF9 which places the mature peptide in a highly bioactive state (McIntosh et al., 2008). In contrast, the prodomain of human GDF9 remains strongly associated following cleavage rendering the mature peptide less bioactive (Mottershead et al., 2008; Simpson et al., 2012). The role of the associated proregion in regulating the activity of each protein appears to vary significantly amongst species (Mottershead et al., 2008; Simpson et al., 2012).

There is evidence to suggest that the proproteins of TGF- $\beta$ family members dimerize before cleavage of the proregion occurs (Hogan, 1996). The efficiency of the dimerization and 
subsequent proteolytic cleavage may be important for regulating the bioactivity of the protein (Constam and Robertson, 1999; Kusakabe et al., 2008). Furthermore, a study with recombinant BMP15 and GDF9 found that coexpression of the two proteins led to impaired cleavage efficiency of the proregion from each protein compared to when each protein was expressed independently. This indicates that the proproteins may form a heterodimeric complex which is less efficiently processed than their homodimeric counterparts (Liao et al., 2003).

As with a number of other TGF $\beta$ proteins, post-translational processing through phosphorylation and/or glycosylation (both $\mathrm{N}$-linked and O-linked) may play an important role in the bioactivity of BMP15 and GDF9 (Hashimoto et al., 2005; McMahon et al., 2008b; Saito et al., 2008). N-linked and O-linked glycosylation has been shown to be important in the function of a range of proteins. For example, O-linked and $\mathrm{N}$-linked oligosaccharides are essential for maintaining the half-life and bioactivity of various glycoprotein hormones, as well as affecting their receptor binding qualities and subsequent signal transduction (Fares, 2006). BMP15 is believed to have five glycosylation sites, with two in the mature region, while GDF9 has four, with one in the mature region (Dube et al., 1998; Hayashi et al., 1999). A study using recombinant human BMP15 and GDF9 demonstrated that both proteins are phosphorylated and that the phosphorylation was essential for their bioactivity (McMahon et al., 2008b). Furthermore, it was shown that the dephosphorylated forms of each protein could have an antagonistic effect toward the phosphorylated form of either protein (McMahon et al., 2008b). While the current evidence points towards an important role in phosphorylation and glycosylation in the function of both proteins, the number of studies is still very limited. 


\subsubsection{Sequence}

Many of the species-specific differences in activity of the two proteins may be explained by small changes in the amino acid sequence between species (Al-Musawi et al., 2013). Sitedirected mutagenesis identified four variable residues in the prodomain that could explain the different levels of expression of BMP15 between humans, sheep and mice, as well as a number of residues which were responsible for determining the species-specific affinity to the type I receptor (Al-Musawi et al., 2013). Moreover, the variation of a single amino acid residue at position 391 in the GDF9 mature region of humans (glycine), compared to mice (arginine), resulted in the protein being secreted in a latent form (with a non-covalently associated prodomain) whilst murine GDF9 was secreted in an active form (Simpson et al., 2012). This residue formed part of the type I receptor binding domain, and the murine form of the residue greatly increased the affinity of the GDF9 for its receptor on granulosa cells. The Gly(391) residue observed in the human form was also found to be present in all other species studied except for a small group of species with high litter size, including rats, mice, and hamsters, and also the mono-ovulatory common brushtail possum, all of which had the high affinity murine form $(\operatorname{Arg}(391))$ (Simpson et al., 2012). These studies indicate that small key differences in the amino acid sequence of GDF9 and BMP15 may explain some of the variation in follicular development and fertility between different mammals.

The genes for both proteins contain two exons flanking a single intron. The signal peptide sequence is encoded on the first exon, the proregion is encoded on portions of both exons, and finally the mature region is found solely on the second exon (Dube et al., 1998; Incerti et al., 1994). The gene for BMP15 is located on the X-chromosome in all mammalian species studied to date whereas GDF9 is autosomally-located (Dube et al., 1998; National Center for Biotechnology Information, NCBI). The autosomal location of GDF9 varies greatly between different mammalian species (for example, chromosome 5 in humans, chromosome 10 in rats, chromosome 11 in mice, chromosome 7 in the cow and chromosome 2 in pigs) (NCBI).

Of the four mammalian species involved in this study (rats, pigs, sheep and deer), BMP15 protein shows the greatest sequence similarity between sheep and deer. Only three residue differences are present in the mature peptide, along with a further three 
differences are in the proregion (see Figure 1.3). The next most homologous species is the pig, with four unique residues in the mature peptide, one in the RXXR cleavage region, and a further sixteen in the proregion. Notable residues that were unique in the pig were in a highly conserved region near the C-terminal end of the mature peptide (two residues), and in the RXXR cleavage region (one residue). The rat has vastly more unique residues than any of the other three species, with 24 in the mature peptide, two in the RXXR cleavage region, and 74 in the proregion. Most notable are two unique residues in the RXXR cleavage region and the clustering of unique residues either side of the cleavage region. The molecular weight of the promature and mature peptides based on the sequence were calculated using the ExPASy software ("ExPASy - Compute pl/Mw tool," 2016). The mature peptide was predicted to be $\sim 13.9-14.0 \mathrm{kDa}$ in all four species, and the promature peptide was predicted to be $\sim 44.9-45.0 \mathrm{kDa}$ in rats, pigs and sheep, but could not be calculated in deer as the full sequence was not available. However, it is likely that the deer promature peptide is approximately the same size given the high homology shared with the sheep sequence. The GDF9 protein (sequence not provided) was predicted to be slightly larger than BMP15 in all four species, with a promature protein between 49.7 and $51.8 \mathrm{kDa}$, and the mature protein around 15.4 to $15.6 \mathrm{kDa}$. These size predictions are based on the sequence alone, and do not take into account any potential post-translational modifications that may occur at potential glycosylation and phosphorylation sites. 


\section{Key}

Residue Variation:

Rat has a unique residue

Pig has a unique residue

Sheep has a unique residue

Deer has a unique residue

Two residues, two species per residu

All residues unique

Residue Properties:

RED- Small, hydrophobic, Aromatic (except Y)

BLUE - Acidic

PURPLE - Basic (except H)

GREEN- Hydroxyl+Sulfhydryl + amine $+\mathrm{G}$

\section{Consensus Symbols:}

* Single fully conserved residue

Strongly similar properties $>0.5$ in Gonnet PAM 250 matrix

Weakly similar properties $=<0.5$ in Gonnet PAM 250 matrix

Promature Region

RXXR Cleavage Site

Mature Region
$\mathrm{N}$-Terminal

RAT MALLTI-LRILLWGMVLFMEHKVQMAKVEWPSTTLLAENPTLPSSLDLAKEAPGKEMK-Q58

PIG MVLLSIIRTLLLWGLVLFMEHRVQMTQVGQPSVALLPEACTLPLIRELLEEAPGKQQRKP 60

SHEEP MVLLSIL-RILLWGLVLFMEHRVQMTQVGQPSIAHLPEAPTLPLIQELLEEAPGKQQRKP 59

DEER

पाताप प

RAT WPQGYPLRYMLKLYQRSADPHGHPRENRTIGAKMVRLIKPSASAMRLLRGPWHIQTLDFP 118

PIG QVLGHPLRYMLELYQRSADARGHPRENRTIGATMVRLVRPLVNGARPLRGPWHIQTLDFP120

SHEEP RVLGHPLRYMLELYQRSADASGHPRENRTIGATMVRLVRPLASVARPLRGSWHIQTLDFP119

DEER

$* * * * * * * * *$

RAT LASNEVAYQLIRATVVYRHQLHLVHYHLSCHVEPWVPKCRTKHFPSK-SGSAKPSSVSKA177

PIG LRPNRVAYQLVRATVVYRHQLHLAPFHLSCHVEPWIQKSTTSHFPSSGRGSLKPSLLPQA180

SHEEP LRPNRVAYQLVRATVVYRHQLHLTHSHLSCHVEPWVQKSPTNHFPSSGRGSSKPSLLPKT179

DEER LRPNRVAYQLVRATVVYRHQLHLTHSHLSCHVEPWIQKSPTSHFPSSGRGSSKPSLLPKA70

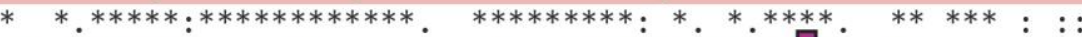

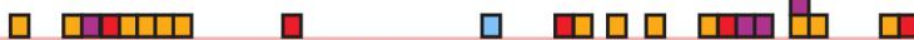

RAT WREMNITHCIQQKLWNRKGRRVLRLRFMCQQQKGNETLELRWHGMTSLDVAFLLLYFNDT 237

PIG WTEMDVTQHVGQKLWNHKGRRVLRLRFMCQQQNGSEILEFRGRGISSLDTAFLLLYFNDT 240

SHEEP WTEMDIMEHVGQKLWNHKGRRVLRLRFVCQQPRGSEVLEFWWHGTSSLDTVFLLLYFNDT 239

DEER WTEMDIMEHVGQKLWNRKGRRVLRLRFMCQQPRGSEVLEFWWHGTSSLDTVFLLLYFNDT130 $* * *::$ : : *****:**********:*** .*.***: :* :***..*********

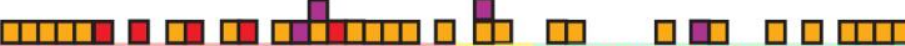

RAT DESAQAKLLARGQEELTDRESPFLMRSVRQTCSIASDVPCPSQEQDRSVNNQCSLHPYKV 297

PIG RSVQKAKLLPRGLEEFMARDPSLLLRKARQAGSIASEVLGPSREHDGPESNd\&SLHPFQV 300

SHEEP QSVQKTKPLPKGLKEFTEKDPSLLLRRARQAGSIASEVPGPSREHDGPESNdCSLHPFQV 299

DEER QSVQKTKPLPKGLKEFTEKDPSLLLRRARQAGSIASEVPGPSREHDGPESNdCSLHPFQV190 . : : * * :* :*: :: :*:* .**: ****:* **:*:* .*******::*

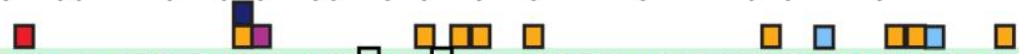

RAT SFHQLGWDHWIIAPRLYTPNYCKGICTGVLPYGLNSPNHAIIQSLVNELVNRSVPQLS $\$ 357$

PIG SFHQLGWDHWIIAPHFYTPNYCKGV CPRVLHYGLNSPNHAIIQNLVNELVDQSVPQPSIV360

SHEEP SFQQLGWDHWIIAPHLYTPNY CKGY CPRVLHYGLNSPNHAIIQNLVSELVDQNVPQPS $\ 359$

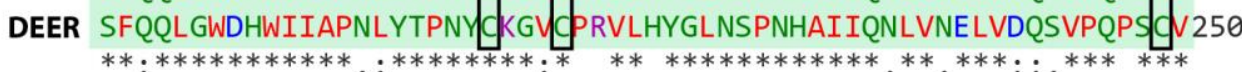

ㅁㅁ민

RAT PYKFLPMSILLIEANGSILYKEYEGMIAQSCTCR 391

PIG PYKYVPISILLIEANGSILYKEYEDMIAQPCTCR 394

SHEEP PYKYVPISILLIEANGSILYKEYEGMIAQSCNCR 393

DEER PYKYVPISILLIEANGSILYKEYEGMIAQSCHCR 284

$* * *:: *: * * * * * * * * * * * * * * * * * * * * * * * * *$

Figure 1.3. The BMP15 sequence in rats, pigs, sheep and deer. The rat, pig and sheep BMP15 sequences were obtained from the UniProtKB database, and primary accession numbers are E9PTU9, Q8WMY6 and Q9MZE2, respectively. The deer sequence was kindly donated by Dr J. Juengel (AgResearch Invermay, NZ). While the rat, pig and sheep sequences shown above span the full promature peptide sequence, the deer sequence only contains peptides from Exon 2 and therefore, is missing a portion of the proregion. The red shaded area corresponds to the proregion for each protein, and the green shaded region represents the mature peptide. There are black borders around the six conserved cysteine residues within the mature peptide which are involved in cysteine knot formation. The yellow segment between the proregion and mature region is the RXXR proprotein convertase target site. The letters for each protein residue are coloured according to their properties. The red residues are all the small, hydrophobic and aromatic amino acids except $Y(A, V, F, P, M, I, L$, and $W)$, whereas the green residues are all the amino acids with hydroxyl, sulfhydryl and amine groups as well as $G(S, T, Y, H, C, N, G$, and $Q)$. The blue residues are the amino acids with acidic side chains ( $D$ and $E$ ), and the purple residues are the 
amino acids with basic side chains except for $\mathrm{H}$ ( $\mathrm{R}$ and $\mathrm{K}$ ). Above the sequence are coloured boxes which represent variation in the amino acid of one or more species away from the most common residue at that point. The yellow box represents variation in the rat residue, the purple box indicates variation in the pig residue, the light blue box indicates variation in the sheep residue and the dark blue box signifies variation in the deer residue. The red box represents a region where there are two different residues, each of which is shared by two species, and the green box represents a region where there all the residues are unique between species. Consensus between the four species at each point is represented by one of three symbols directly under the sequence. The asterisk $(*)$ indicates that all four species share the same residue, the colon (:) indicates that they vary but share strongly similar properties, and the period (.) indicates that they vary but share weakly similar properties. The lack of any of the three symbols indicates that as a whole, the residues of all four species are highly varied at that point.

\subsubsection{Signalling Pathways}

As with all TGF- $\beta$ family proteins, both BMP15 and GDF9 act on their target cells via a receptor complex made up of type I and type II receptor Serine/Threonine kinases (as described in Section 1.1.3) (Paulini and Melo, 2011). Within the receptor complex, the type II receptors are constitutively active, and once they bind the ligand they recruit, phosphorylate and activate the type I receptors. The Type I receptors then phosphorylate and activate certain receptor-specific Smad proteins (rSmads), which in turn translocate into the nucleus and regulate the transcription of certain genes (Juengel et al., 2004; McNatty et al., 2004).

The mature region of BMP15 binds a complex consisting of the BMP type II receptor (BMPRII) and the type I receptor ALK6/BMPR1B. The binding and subsequent phosphorylation of ALK6 by BMPRII activates the Smads 1, 5 and 8 within the cell (Moore et al., 2003). The mature region of GDF9 also binds BMPRII however its binding recruits the type I receptor ALK5. This BMPRII/ALK5 complex signals through the Smads 2 and 3 (Mazerbourg et al., 2004). The Smad $1 / 5 / 8$ and Smad $2 / 3$ pathways both involve cooperation with Smad4 before translocating into the nucleus and regulating the expression of certain genes associated with proliferation, expansion and luteinisation, such as pentraxin 3 (Ptx3), hyaluronan synthase 2 (Has2), and prostaglandin-endoperoxide synthase 2 (Ptgs2) (Mazerbourg et al., 2004; Moore et al., 2003; Peng et al., 2013a). As with some other members of the TGF- $\beta$ superfamily, there is evidence that both BMP15 and GDF9 may also signal through the MAPK pathway, however this varies between species 
(Moore et al., 2003; Reader et al., 2011). The epidermal growth factor receptor (EGFR)extracellular signal-regulated kinase 1 and 2 (ERK1/2) may be required by GDF9 alongside Smad2/3 for proper signalling to occur as ERK1/2 helps maintain Smad3 phosphorylation (Sasseville et al., 2010). It was believed that the synergistic interaction of BMP15 and GDF9 together may involve the ERK1/2 (also known as MAPK3/1) signalling pathway alongside the Smad pathway (Mottershead et al., 2012). However, inhibitors of the ERK/MAPK and the p38-MPAK pathway did not affect the synergistic action of human GDF and BMP15 on granulosa cell proliferation rate so this appears unlikely (Reader et al., 2014). Other pathways such as the SRC kinase pathway also may play a role in the synergistic interaction of the two proteins (Mottershead et al., 2012).

In the case of human and mouse granulosa cells, the potent action of the BMP15:GDF9 heterodimer on follicle development is believed to work through a receptor complex of BMPRII receptors, together with an ALK 4/5/7 type I receptor and an ALK6 type I coreceptor (Peng et al., 2013a). Binding of the heteromeric dimer causes the phosphorylation of Smads 2/3 in both species despite BMP15 homodimers being shown to signal through the Smad1/5/8 pathway (Mazerbourg et al., 2004; Moore et al., 2003; Peng et al., 2013a). Other studies have demonstrated that the MAPK signalling pathway may also be involved in signal transduction alongside the Smad 2/3 pathway (Mottershead et al., 2012). More recent studies using BMP15 and GDF9 heterodimers of both the promature and mature proteins (termed pro-cumulin and cumulin, respectively) have indicated they activate both the Smad2/3 and the Smad 1/5/8 signalling pathways within granulosa cells (Mottershead et al 2015). 


\subsubsection{Biological Effects}

\subsubsection{Genetics Studies}

There have been extensive murine genetic knockout studies that investigated the role of BMP15 and GDF9 in fertility. The GDF9 null mice $\left(\right.$ Gdf $\left.9^{-/}\right)$are completely infertile, with anovulation being a key characteristic of the genotype (Dong et al., 1996; Yan et al., 2001). On the other hand, homozygous BMP15 knockout mice $\left(B m p 15^{-}\right)$only present a minor loss of fertility. Bmp15 $15^{+-}$mice have a normal fertility phenotype, while $\mathrm{Gdf9}^{+/-}$mice have an increased ovulation and cumulus cell expansion rate, and subsequently have increased fertility (Su et al., 2004). Interestingly, when a Bmp15\% genotype is combined with a $\mathrm{Gdf9}^{+/-}$ genotype, there are severe defects in the ovary and the mice are essentially sterile, with reduced production of proteins associated with cumulus cell expansion, and impaired follicle and oocyte development (Su et al., 2004; Yan et al., 2001). These studies suggest that both BMP15 and GDF9 play a role in fertility outcomes in mice, however BMP15 plays a minor role compared to GDF9. These observations also support the possibility of a functional interaction between the two proteins in vivo (Dong et al., 1996; Su et al., 2004; Yan et al., 2001).

Various mutations in BMP15 and GDF9 genes have been discovered in sheep, and have subsequently been used in a range of studies to evaluate the role of the proteins in a low ovulatory species. Homozygous nullifying mutations within the $B M P 15$ gene $\left(B M P 15^{--}\right)$in ewes causes complete infertility by halting follicle development beyond the primary stage. However, heterozygotes $\left(B M P 15^{+/}\right)$with the same mutation have an increased ovulation rate and are more fertile than their wildtype counterparts (Galloway et al., 2000). This contrasts to that observed in $B M P 15^{-/-}$and $B M P 15^{+/-}$mice, as described above (Galloway et al., 2000; Su et al., 2004; Yan et al., 2001). Similar observations were made in highly fertile sheep lines with non-conservative knockout mutations in either BMP15 or GDF9 (Hanrahan et al., 2004). The $B M P 15^{+/-}$and $G D F 9^{+/-}$genotypes resulted in an increased ovulation rate in ewes, however BMP15\% OR GDFg\% caused sterility, highlighting the equal importance of both GDF9 and BMP15 in sheep (Hanrahan et al., 2004). Interestingly, GDF9+/-/BMP15+/- 
sheep exhibited an even greater ovulation rate than ewes that had a heterozygous mutation in either protein alone (Hanrahan et al., 2004).

In humans, studies into genetic mutations in the BMP15 gene have shown that abnormalities in the protein may play a role in hypergonadotropic ovarian failure, primary ovarian insufficiency, premature ovarian failure, and the ovarian phenotype of women with Turners Syndrome (Di Pasquale et al., 2004; Laissue et al., 2006; Persani et al., 2014; Rossetti et al., 2009). BMP15 may have a role in polycystic ovarian syndrome (PCOS) however this remains controversial (Persani et al., 2014). Studies into genetic mutations in GDF9 suggest it could play a role a number of reproductive disorders such as PCOS, diminished ovarian reserve, premature ovarian failure and dizygotic twinning (Laissue et al., 2006; Persani et al., 2014; Wang et al., 2010, 2013). These studies suggest that secretion of adequate amounts of bioactive forms of both BMP15 and GDF9 may also be essential for maintaining proper reproductive function in humans.

\subsubsection{In vitro Bioassays}

Recombinant BMP15 from a range of species has been used in numerous in-vitro studies to investigate its biological effects. The role of BMP15 in rats was investigated using a rat granulosa cells (GC) bioassay. Recombinant BMP15 added to rat GC showed that BMP15 was a strong stimulator of GC proliferation in a manner independent of follicle stimulating hormone (FSH) (Otsuka et al., 2000). It also caused a significant decrease in FSH-induced progesterone production, but not FSH-induced oestradiol production, suggesting BMP15 can selectively regulate FSH action (Otsuka et al., 2000). The mechanism for this regulation may be the down-regulation of the FSH receptor (Otsuka et al., 2001). A similar effect of down-regulation of FSH-induced progesterone production was observed in sheep (Fabre et al., 2006).

A study using recombinant human BMP15 and human GCs demonstrated that it also increases GC proliferation in a dose-dependent manner (Di Pasquale et al., 2004). Ovine BMP15 was shown to stimulate the proliferation of both ovine and bovine GCs, while not stimulating rat GCs, suggesting that the GCs of different species interact with BMP15 in a 
species-specific manner (McNatty et al., 2005a, 2005b). Ovine BMP15 and GDF9 combined were shown to have a synergistic effect in sheep, however in cows both proteins combined were no different at stimulating GC proliferation than ovine BMP15 alone (McNatty et al., 2005a). In another study using mouse follicles, BMP15 or GDF9 alone was shown to promote pre-antral follicle growth in vitro during the first 24 hours of culture (Fenwick et al., 2013). However, between 48 and 72 hours of culture, the addition of BMP15 alone was found to cause follicle shrinkage and atresia as well as increased apoptosis of granulosa cells. Interestingly, when GDF9 was added alongside BMP15, the shrinkage and atresia effects were eliminated, even with culture beyond 24 hours, and instead there was increased growth beyond what was seen with the addition either protein alone (Fenwick et al., 2013). An ALK6 inhibitor blocked the effects of BMP15 while an ALK5 inhibitor blocked the effects of both GDF9 and the combined effects of GDF9 with BMP15 (Fenwick et al., 2013).

\subsubsection{In vivo Bioassays}

Ewes immunized long-term against peptides specific to either BMP15 or GDF9 tended to show anovulation and abnormal follicle development, supporting the hypothesis that both proteins are essential for healthy follicle development in mono-ovulatory mammals (Juengel et al., 2002). Moreover, it was later found that short-term immunisation against BMP15 or GDF9 specific peptides increased the ovulation rate in immunized ewes (Juengel et al., 2004). It was also shown that immunisation at different regions of the mature peptide for each protein had different fertility outcomes, whereby immunization against the $\mathrm{N}$ terminal region tended to induce anovulation whereas immunization against the central portion could increase ovulation rate (McNatty et al., 2007). A similar immunization study carried out in cattle demonstrated the importance of both proteins in follicle development and ovulation, however the immunisations were less effective than in sheep, possibly highlighting differences in the roles of each protein between species (Juengel et al., 2009).

Similar immunization approaches against the proregion of BMP15 in mice caused fewer corpora lutea to form in the ovaries and smaller litter sizes, while immunisation against 
GDF9 caused more corpora lutea to form, and a substantial drop in litter size (McIntosh et al., 2012). These results suggest that the proregions of BMP15 and GDF9 are important in mouse fertility outcomes. Transgenic mice which were created to overexpress mature human BMP15 in the oocytes were found to have accelerated follicle growth, an increase in the proliferation of granulosa cells, a decrease in granulosa cell FSH receptor mRNA expression, and an early decline in ovarian follicle reserve (McMahon et al., 2008a). The findings of the study suggest BMP15 promotes follicle growth while preventing follicle maturation in mice, which may be why BMP15 is found in such low levels in polyovulatory species such as mice (McMahon et al., 2008a). 


\subsection{Significance of the Study}

The number of offspring produced in a single litter varies significantly between different mammalian species. The litter size is dependent upon the number of eggs released during ovulation and the developmental competence of each oocyte. BMP15 has been shown to influence ovulation rate as well as developmental competency of the oocyte by regulating granulosa and cumulus cell function during development, including through interactions with the closely-related GDF9 protein (Paulini and Melo, 2011). It has been almost two decades since BMP15 was first discovered, and in that time a vast number of studies across a range of different mammalian species have corroborated the idea that BMP15 is a key factor in determining fertility outcomes through roles that are specific to each species (Persani et al., 2014). Further research into the mechanisms behind follicular development and fertility is important for improving our understanding of the causes behind a range of fertility disorders, and may eventually lead to potential therapeutic options that target the pathways involved (Persani et al., 2014). Furthermore, an improved understanding may also be useful in improving the success rate of assisted reproductive technologies such as IVF and IVM, which currently have highly variable and often unsuccessful outcomes (Gilchrist, 2011; Mottershead et al., 2015; Persani et al., 2014).

Whilst a substantive body of research has been collected over the past decade on BMP15, its actions on, and molecular forms in, different species with a range of litter sizes are unknown. Almost all of the current research into its molecular forms has involved the use of recombinant proteins, rather than investigating the native forms. Knowledge of the native forms could provide more accurate information on species specific-differences in molecular forms, and the relative amounts of each form of the protein produced in a more natural setting. One study has looked at the molecular forms of rat and sheep BMP15 in oocyte lysate and oocyte conditioned media (Lin et al., 2012). This current study aims to corroborate what has been reported previously regarding the forms of BMP15 in rats and sheep, as well as investigate the molecular forms and relative amounts of BMP15 in two new species, namely pigs and red deer. Pigs are of particular interest because they are a non-rodent species with a high litter size. Moreover, the pig is the only species studied which does not match the trend whereby a high ratio of GDF9:BMP15 relates to a large 
litter size (Crawford and McNatty, 2012). In pigs, the ratio of GDF:BMP15 mRNA produced is a relatively low 0.51 , despite having a large litter size of $14-22$ offspring (Crawford and McNatty, 2012). Information on the native molecular forms of BMP15 may provide clues as to why this is. The red deer is a strict monovulator, more so than the sheep, and will provide a good comparison for the pig. Furthermore, comparisons of the native molecular forms with previously published information on the recombinant molecular forms will provide insight into the accuracy of the use of recombinant forms as models of the proteins in a native setting. 


\subsection{Aims}

Aim 1: To characterise and optimise a novel monoclonal antibody (Mab61B) for detecting the different molecular forms of BMP15 across different mammalian species, and to compare these results with a previously-characterised monoclonal antibody (Mab61A) that has proven effectiveness both in recognising different molecular forms, and in providing immunosuppression of native forms of BMP15.

To address this aim, Western blots were performed with the Mab61B monoclonal antibody under various conditions using both recombinant and native forms of BMP15 from a range of mammalian species until optimal signal was obtained. The protein forms detected under optimal conditions were then compared to those which could be detected by the fully-characterised Mab61A antibody in order to determine which antibody would be best for use in this study.

Aim 2: To determine the native molecular forms of BMP15 that were present both in the oocyte (intra-oocyte) and secreted from the oocyte (oocyte-secreted) in a range of different mammalian species with different litter sizes.

To address this aim, Western blots were performed using oocyte lysates and oocyte-conditioned media from pigs, red deer, rats and sheep, which have litter sizes of 14-22, 1, 7-16 and 1-3, respectively. Western blots were performed under non-reducing and reducing conditions, and included cross-linked forms under reducing conditions to provide insight into the forms that exist and the strength of any potential dimer or multimer formations. These blots were then stripped and probed with an antibody targeting GDF9 to determine if any of the molecular forms detected with the anti-BMP15 antibody were in a complex with the highly homologous GDF9 protein.

Aim 3: To determine the differences in the relative amounts of each of the molecular forms of BMP15 present, both within and between species.

To address this aim, densitometric analysis was performed on the bands that represented the different forms of BMP15 detected in the Western blots. The 
relative amounts of the different forms between species were normalised to either the amount of a constitutively-active reference protein ( $\beta$-actin), or to the number of oocytes used for each species to elucidate the best normalisation procedure. Finally, to account for inter-blot variation when the blots were normalised to the number of oocytes, the different molecular forms within each blot were also normalised against the positive control (recombinant pig mature BMP15 band) present in each blot. 


\section{Chapter 2: Materials and General Methodology}

\subsection{Methods}

\subsubsection{Ovary Collection}

Rat ovaries were collected from prepubertal Sprague-Dawley rats aged between 3 and 4 weeks. The rats were sacrificed by asphyxiation with $\mathrm{CO}_{2}$ gas followed by cervical dislocation which was a standard operating procedure that had been approved by the VUW animal ethics committee. The ovaries were removed and placed into in a $15 \mathrm{~mL}$ centrifuge tube containing M199 collection media (Sigma-Aldrich) at room temperature. Once all ovaries had been collected, they were taken a short distance to the lab and transferred to a 30mm petri dish with fresh M199 collection media. Excess connective tissue surrounding the ovary was then carefully removed under the Leica MZ9S stereo microscope using dissection scissors.

Sheep ovaries were collected from lambs and ewes of unknown background from the Taylor Preston meat processing plant in Ngauranga, Wellington. The ovaries were transported back to the laboratory on ice. Excess tissue was then trimmed from each ovary, and they were washed for 10 seconds in $70 \%$ ethanol to remove any potential bacterial contamination before being thoroughly rinsed with $0.9 \%$ saline solution. The ovaries were kept in $0.9 \%$ saline solution at room temperature before being transferred to M199 collection media in a $100 \mathrm{~mm}$ petri dish for oocyte collection.

Deer ovaries were collected from red deer of unknown background from the Venison Packers Feilding Ltd meat processing plant in Fielding. The ovaries were washed with $0.9 \%$ saline before being placed in fresh $0.9 \%$ saline in a vacuum flask and transported for 3 hours back to the laboratory.

Pig ovaries were collected from farmed pigs of unknown background from the production line at Cabernet Foods, Kintyre Meats Ltd in Gladstone. The ovaries were washed (as 
described above for the deer ovaries) and transported for 2 hours back to the laboratory. Back in the laboratory, the deer and pig ovaries were trimmed and washed, before being transferred to M199 collection media in a $100 \mathrm{~mm}$ petri dish for oocyte collection.

Ovaries from each species were collected throughout the year between April and October. Ovaries with obvious Corpora lutea were not used for oocyte collection. Oocyte samples were collected across multiple days and the samples from each species used for each experiment were selected at random.

\subsubsection{Oocyte collection}

To collect oocytes from rats, rat ovaries were placed in a $60 \mathrm{~mm}$ petri dish containing M199 collection media under a dissecting microscope. The ovaries were punctured repeatedly with a $26 \mathrm{G}$ precision glide needle until it appeared that all of the follicles had been ruptured. The ovary was then discarded. A maximum of five ovaries were processed per dish. The media containing the ovaries was then very slowly pulled through a 206 needle into a $5 \mathrm{~mL}$ syringe before being injected very gently into a $40 \mu \mathrm{m}$ cell strainer. The oocytes were then carefully washed off the cell strainer into a new $30 \mathrm{~mm}$ petri dish by inverting the strainer and injecting fresh M199 collection media through it. Once the cells had been allowed to settle, rat cumulus cell-oocyte complexes (COCs) and denuded oocytes (oocytes free of any cumulus cells) were collected and transferred to another $30 \mathrm{~mm}$ petri dish with fresh M199 collection media using a $10 \mu \mathrm{L}$ Gilson pipette.

Once all COCs and denuded oocytes were collected, the fully denuded oocytes were transferred into $1.5 \mathrm{~mL}$ microcentrifuge tubes containing fresh M199 collection media in aliquots depending on the number of oocytes required (200 for oocyte lysate samples and 250 for oocyte-secreted media). The remaining COCs were then gently drawn into a $5 \mathrm{~mL}$ syringe via a $20 \mathrm{G}$ needle and reinjected back into the petri dish several times to detach the cumulus cells. Once fully or partially denuded, the oocytes were transferred into the 1.5 $\mathrm{mL}$ microcentrifuge tubes. Partially denuded oocytes were accepted as it was difficult to remove the last few cumulus cells $(<10)$ without damaging the oocyte. Only healthy 
oocytes, as assessed by morphology, with an intact cell membrane were transferred. Once all oocytes were transferred, the microcentrifuge tubes were centrifuged at $500 \mathrm{~g}$ for 4 minutes at room temperature. The supernatant was carefully discarded using a pipette under a dissecting microscope, and the oocytes were then resuspended in fresh M199 collection media. The tubes were once again centrifuged at $500 \mathrm{~g}$ for 4 minutes and then the supernatant removed. At this stage, the oocytes intended for use as a lysate sample had $1 \mu \mathrm{L}$ of $10 \mathrm{X}$ protease inhibitor solution added and were vortexed vigorously before being centrifuged for 1 minute at $7000 \mathrm{~g}$. The oocyte lysate samples were then snap-frozen by placing the tubes in dry ice, and the tubes were stored at $-80^{\circ} \mathrm{C}$ until required. The oocytes that were intended for incubation to provide the oocyte-secreted media samples were resuspended in $25 \mu \mathrm{L}$ of M199 incubation media and placed in an incubator at $37^{\circ} \mathrm{C}$ ( $5 \% \mathrm{CO}_{2}, 95 \%$ humidity) for 18 hours. Following incubation, the tubes were centrifuged at $500 \mathrm{~g}$ for $4 \mathrm{~min}$ and the conditioned media (media containing the native BMP15 secreted from the oocytes) was transferred to a new $1.5 \mathrm{~mL}$ microcentrifuge tube, carefully avoiding the transfer of any cells. The incubated oocytes used to provide the secreted media were discarded and not used in subsequent experiments. An aliquot of $2.5 \mu \mathrm{L}$ of $10 \mathrm{X}$ protease inhibitor solution was added to the conditioned media and it was stored at $-80^{\circ} \mathrm{C}$ until required.

Sheep and deer oocytes were collected by first placing the ovaries in a $600 \mathrm{~mm}$ petri dish partially-filled with M199 collection media and then slicing them in half through the hilum along the midline. A maximum of two ovaries were processed per petri dish. The halves of each ovary were punctured using a $20 \mathrm{G}$ needle fixed to a $5 \mathrm{~mL}$ syringe until all healthy follicles were ruptured. The fully-punctured ovaries were then discarded. Once oocytes had been removed from all ovary halves, the media was gently drawn into the $5 \mathrm{~mL}$ syringe via the $20 \mathrm{G}$ needle and then reinjected into the petri dish several times to partially denude the oocytes and break up any other cell debris present. The media was once again drawn into the syringe and then gently injected into a $100 \mu \mathrm{M}$ cell strainer. The cells collected in the cell strainer were washed off into a new $60 \mathrm{~mm}$ petri dish using fresh M199 collection media. The COCs and denuded oocytes were then transferred to fresh media in a $30 \mathrm{~mm}$ petri dish using a $10 \mu \mathrm{L}$ Gilson pipette. The fully denuded oocytes were then transferred into microcentrifuge tubes containing fresh M199 collection media. The remaining COCs 
were fully or partially denuded using a syringe in the same manner as the rat oocytes before being transferred to the microcentrifuge tubes. Final aliquots for the sheep oocytes were either 100 oocytes per tube for the oocyte lysate samples, or 125 oocytes per tube for the oocyte-secreted media samples. The oocyte aliquots were washed and then either frozen or incubated using the method previously described above. However, one modification was that the temperature used to incubate the ovaries was $39^{\circ} \mathrm{C}$, rather than $37^{\circ} \mathrm{C}$, to more closely resemble the body temperature of sheep and deer. All samples were stored at $-80^{\circ} \mathrm{C}$ until required.

Pig oocytes were collected in a very similar manner to sheep and deer oocytes; however there were a few points of difference. The ovaries were not sliced in half prior to being punctured with a needle as the abundance of follicles rendered it unnecessary, and a $70 \mu \mathrm{M}$ strainer was used rather than a $100 \mu \mathrm{M}$ strainer due to the smaller oocyte size. The oocytes were collected in aliquots of 80 oocytes for the cell lysate samples, and 100 oocytes for the incubation samples. Also, the physical denudation stage using the syringe was not required as the pig oocytes tended to leave the follicles in a fully denuded state when they were punctured.

\subsubsection{Western blotting}

\subsubsection{Preparation of SDS-PAGE gel}

The polyacrylamide separating gels were made using either $13.5 \%, 15 \%$ or $7.5 \%(\mathrm{v} / \mathrm{v})$ acrylamide/bis solution, and each separating gel was combined with a $4 \%$ acrylamide stacking gel (see Table 2.1). The gels were prepared using $0.75 \mathrm{~mm}$ Mini-PROTEAN casting module (Bio-Rad Laboratories, Cat\#1658008) as part of the Mini-Protean Tetra Cell electrophoresis kit (Bio-Rad Laboratories, Cat\# 165-8000). The reagents in the separating gel at the desired acrylamide percentage (Table 2.1) were combined in a glass beaker before being added to the glass casts secured on the casting module. A thin layer of High$Q$ water was added to the top of the separating gel mixture in each cast to prevent the gel from drying during polymerisation. The gel was then left for at least 1 hour at room 
temperature to polymerise. Once polymerisation was complete, the thin water layer was removed and the $4 \%$ stacking gel solution was carefully added on top of the separating gel. At this stage, a $0.75 \mathrm{~mm}$ gel comb (10 or 15 wells depending experiment) was inserted into the stacking gel solution, and the stacking gel was left at room temperature for a further $\sim 30$ minutes to polymerise. Once the stacking gel was polymerised, the comb was either removed, and the gel used immediately, or the comb was left in place and the gels were stored at $4^{\circ} \mathrm{C}$ in a sealed plastic back covered in wet paper towels for future use.

Table 2.1: Separating and stacking gel recipes (makes two separating and 4 stacking gels)

\begin{tabular}{|c|c|c|c|c|}
\hline Reagents & $\begin{array}{c}\mathbf{1 5 \%} \\
\text { Separating gel }\end{array}$ & $\begin{array}{c}\mathbf{1 3 . 5 \%} \\
\text { Separating gel }\end{array}$ & $\begin{array}{c}\mathbf{7 . 5 \%} \\
\text { Separating gel }\end{array}$ & $\begin{array}{c}\mathbf{4 \%} \\
\text { Stacking gel }\end{array}$ \\
\hline High-Q distilled water & $2.35 \mathrm{~mL}$ & $2.85 \mathrm{~mL}$ & $4.85 \mathrm{~mL}$ & $3.0 \mathrm{~mL}$ \\
\hline $\mathbf{1 . 5 M}$ Tris-HCl pH8.8 & $2.5 \mathrm{~mL}$ & $2.5 \mathrm{~mL}$ & $2.5 \mathrm{~mL}$ & $0 \mathrm{~mL}$ \\
\hline $\mathbf{0 . 5 M}$ Tris-HCl pH6.8 & $0 \mathrm{~mL}$ & $0 \mathrm{~mL}$ & $0 \mathrm{~mL}$ & $1.25 \mathrm{~mL}$ \\
\hline $\begin{array}{c}\text { Bis-acrylamide mix } \\
(\mathbf{3 0 \% )}\end{array}$ & $5.0 \mathrm{~mL}$ & $4.5 \mathrm{~mL}$ & $2.5 \mathrm{~mL}$ & $0.65 \mathrm{~mL}$ \\
\hline $\mathbf{1 0 \% ( w / v ) ~ S D S}$ & $0.1 \mathrm{~mL}$ & $0.1 \mathrm{~mL}$ & $0.1 \mathrm{~mL}$ & $50 \mu \mathrm{L}$ \\
\hline $\begin{array}{c}\mathbf{1 0 \%}(\mathbf{w} / \mathbf{v}) \text { ammonium } \\
\text { persulphate }\end{array}$ & $50 \mu \mathrm{L}$ & $50 \mu \mathrm{L}$ & $50 \mu \mathrm{L}$ & $25 \mu \mathrm{L}$ \\
\hline TEMED & $5 \mu \mathrm{L}$ & $5 \mu \mathrm{L}$ & $5 \mu \mathrm{L}$ & $5 \mu \mathrm{L}$ \\
\hline
\end{tabular}

\subsubsection{Sample Preparation}

Samples required for each gel were thawed, vortexed well and then centrifuged briefly for removal of cellular debris. In order to keep the sample volume constant between wells for a given blot, additional M199 incubation media was added to as required to those samples with less volume such that they matched the highest sample volume for the blot. At this stage, if a given sample type was to be compared across several blots under different conditions, such as an oocyte lysate sample under reducing and non-reducing conditions; the samples were combined in one microcentrifuge tube, vortexed briefly, and then reseparated into individual tubes to ensure that the sample was homogeneous between blots. Next, if a sample was required to be crosslinked, freshly prepared $14 \mathrm{mM}$ (7Xstrength) BS3 crosslinking solution was added to give a final concentration of $2 \mathrm{mM}$ in the sample, and the sample was vortexed briefly before being left at room temperature for 30 minutes to allow the non-cleavable crosslinking reaction to occur. Double-strength 
modified Laemmli loading buffer (either reducing or non-reducing, see Appendix I; Table 3) was then added to each sample in a 1:1 ratio and the samples were denatured in a water bath at $95^{\circ} \mathrm{C}$ for 5 minutes. Unless it is stated that a non-reducing loading buffer was used, it can be assumed that a reducing loading buffer was used. The samples were then centrifuged at $1000 \mathrm{~g}$ for 2 minutes using a Minispin Plus centrifuge to remove any insoluble particles, and loaded onto the gel. Different ladders were used in different gels depending on the protein of interest and availability. The SDS-PAGE broad range ladder (Bio-Rad) was combined with an equal volume of double-strength modified Laemmli reducing loading buffer (see Appendix I; Table 3) and denatured in a water bath at $95^{\circ} \mathrm{C}$ for 5 minutes. Where the Kaleidoscope prestained SDS-PAGE standards and the Precision Plus Protein Dual Color standards (Bio-Rad) were used, these reagents were utilised at room temperature without heating or the addition of a loading buffer as per the manufacturer's directions.

\subsubsection{Gel Electrophoresis and Transfer}

The gels were placed in a Mini-Protean Tetra Cell running cassette (Bio-Rad Laboratories, Cat\# 165-8000) and secured in place. When an odd number of gels were being run, a buffer dam (Bio-Rad Laboratories, Cat\# 165-3130) was used. The cell was filled with running buffer (5x running buffer diluted to $1 \mathrm{X}$ with High-Q water, see Appendix I, Table 3) according to the manufacturer's directions, and the gels were electrophoresed for 1-1.5 hours at 150V until the loading dye had run out of the bottom of the gel.

The protein in the gels was then transferred to nitrocellulose membranes (Amersham Protran, GE Healthcare, Cat\# 10600004) using a Criterion ${ }^{\mathrm{TM}}$ Blotter with plate electrodes (Bio-Rad Laboratories, Cat\# 170-4070). To prepare the transfer cassette for protein transfer, a gel/blot assembly tray was filled with transfer buffer (see Appendix I, Table 3). Four pieces of gel blotting paper and one piece of nitrocellulose membrane were trimmed to approximately $10 \mathrm{~cm} \times 7.5 \mathrm{~cm}$ per each gel to be transferred. The blotting paper, nitrocellulose membrane and two foam pads were soaked in transfer buffer and stacked within the transfer cassette in the following order from top to bottom: 
Top

Red side of cassette (positive charge)

Foam pad

2 pieces of blotting paper

Membrane

Gel (removed from glass cast and stacking gel discarded)

2 pieces of blotting paper

Foam pad

Black side of cassette (negative charge)

Bottom

As each layer was added, a roller was used to remove any air bubbles, and tweezers were used when handling the membrane to avoid any contamination. Care was taken to ensure the membrane did not move once in contact with the gel. The assembled cassette was clasped together and then placed into the Criterion Blotter along with and ice pack (frozen at $-20^{\circ} \mathrm{C}$ ) and a magnetic stir bar. It was then filled with transfer buffer (stored at $4^{\circ} \mathrm{C}$ ) until the cassette was submerged, and then the whole device was placed in an ice bath over a magnetic stirrer. The transfer was then carried out for 1 hour at $100 \mathrm{~V}$. Once the transfer was complete, the membrane was removed from the cassette and the gel carefully separated from the membrane and discarded. The membrane was then trimmed to the gel area, and the ladder was lightly marked with a pencil. The membranes were stained with Ponceau S stain (see Appendix I, Table 3) for 1 minute, and then rinsed briefly with distilled water until the protein bands were visible to demonstrate a successful transfer. If the lanes on the membrane were to be divided for multiple antibody incubations, this was done at this point. The Ponceau S stain was then fully removed from the membrane by washing with membrane wash buffer (see Appendix I, Table 3) for $2 \times 10$ minutes on a see-saw rocker set at 15 tilts/min. The membrane was then placed in a plastic container (Uniware, see Appendix I, Table 1) and incubated for 1 hour in blocking buffer under agitation via a low speed Orbit LS shaker set at 60rpm at room temperature. The blocking buffer used contained 5\% skim milk powder unless stated otherwise (see Appendix I Table 3). 


\subsubsection{Immunodetection of target proteins}

Once incubation with the block buffer was complete, the buffer was discarded and the primary antibody solution was added to each membrane. Different primary antibodies were used for different experiments; however every antibody used was diluted in blocking buffer to make the primary antibody solution. Mab61A (anti-BMP15) and Mab37A (antiGDF9) were used at $1 \mu \mathrm{g} / \mathrm{ml}$ in all experiments while Mab61B (Anti-BMP15) was used at either $1 \mu \mathrm{g} / \mathrm{ml}$ or $2 \mu \mathrm{g} / \mathrm{ml}$ (see Appendix I, Table 4 for antibody details). The mouse monoclonal $\beta$-Actin primary antibody (Sigma-Aldrich, cat\# A5441) was used at a 1:5000 dilution. The membrane was incubated for 12 hours in primary antibody solution at $4^{\circ} \mathrm{C}$ on a low speed Orbit LS shaker set at 60rpm unless stated otherwise. Thereafter, the membrane was washed $3 \times 10$ minutes with membrane wash buffer (see Appendix I, Table 3) on a see-saw rocker set at 15 tilts/min. Following the wash step, the membranes were incubated with the secondary antibody solution (Peroxidase-conjugated Affinipure Rabbit Anti-mouse IgG, Jackson ImmunoResearch Laboratories Inc). Unless stated otherwise, the secondary antibody was diluted 1:2000 in blocking buffer to a final concentration of 0.2 $\mu \mathrm{g} / \mathrm{mL}$. The membrane was incubated for $2-5$ hours (depending on the experiment) at room temperature on a low speed Orbit LS shaker set at 60rpm. In the case of blots using oocyte lysates and conditioned media, a 5 hour incubation time was used. The membrane was then washed $3 \times 10$ minutes with membrane wash buffer (Appendix I, Table 3) on a seesaw rocker set at 15 tilts/min. The membrane wash buffer was then discarded before immediately proceeding with chemiluminescent detection. If chemiluminescent detection could not be immediately performed, for instance if multiple blots needed to be imaged separately, then the blots were kept in membrane wash buffer at $4^{\circ} \mathrm{C}$ for up to 2 hours before proceeding with the protein detection to prevent the membrane from drying out.

\subsubsection{Chemiluminescent Detection}

Chemiluminescent detection was used to visualise the labelled proteins on the membrane. First, all membrane wash buffer was poured off the membrane and the membrane was sprayed 3 to 4 times with the WesternBright ECL-Spray (Western Blotting Detection System; Advansta). Once sprayed, the membrane was gently rocked from side to side for 2 
minutes before removing the membrane from the spray solution with tweezers, and allowing the majority of the spray to drip off, before placing the membrane on a piece of $3 \mathrm{M}$ transparency film. The membrane and transparency film were then placed inside an Omega Lum G Imaging System (Aplegen). The membranes were imaged using the Chemi HD settings at various exposure times and cumulative exposures depending on the experiment. If a given blot was going to be directly compared with other blots, the exposure length was kept constant. The oocyte lysate and oocyte-conditioned media blots for each species under non-reducing, reducing and crosslinking conditions were all exposed for $1 \times 10$ min to ensure full comparisons could be made. The number of exposures and the exposure length used was chosen to provide the strongest possible signal of all bands present without any of the bands being saturated. In some cases, multiple different exposures were taken from the same blot so that results were available at optimal exposure lengths for both the dense and the faint bands on the same blot.

All images from the blots were saved in their raw TIFF file format. This was used for densitometric analysis. Images were also saved in JPEG format so that if visual comparisons were made between the images of different blots, the black, white and gamma levels could be kept constant. Once all the required chemi HD images had been saved, the same membranes were then imaged using the epiwhite setting on the Omega Lum $\mathrm{G}$ imaging system, with the exposure set to automatic. This image was saved as a JPEG format and was used to visualise the ladder which had been marked with pencil earlier in the process, and was later used to add the ladder to the corresponding chemi HD chemiluminescent images. The membranes which were required for further experiments were then placed in a fresh plastic container (Uniware) and were washed for $2 \times 10$ min with membrane wash buffer before proceeding to the membrane stripping protocol for antibody removal. Membranes not required for future experiments were discarded. 


\subsubsection{Membrane Stripping}

A stripping buffer was prepared in a Duran glass bottle (see Appendix I, Table 3) and preheated to $50^{\circ} \mathrm{C}$ in a water bath. The membranes to be stripped were placed in $200 \mathrm{~mL}$ sealable Klip It ${ }^{\mathrm{TM}}$ containers (Sistema) and sufficient membrane stripping buffer was added to provide approximately $1 \mathrm{~cm}$ of cover over the entire membrane. This was then placed in the $50^{\circ} \mathrm{C}$ water bath for 30 minutes, with gentle agitation every 5 minutes. The stripping buffer was then discarded from the container, and the membrane was washed $3 \times 10$ minutes with large volumes of membrane wash buffer. The membrane was then blocked for 1 hour in blocking buffer containing $5 \%(w / v)$ skim milk powder unless stated otherwise. Immunodetection and chemiluminescent detection was then performed as in Sections 2.1.3.4 and 2.1.3.5.

\subsubsection{Antibody preadsorption}

Primary antibody solutions of both Mab61A and Mab61B for preadsorption were prepared 24 hours prior to use. The primary antibody concentrations have been previously described (see Section 2.1.3.4). The blocking peptide (either Peptide B, E.coli-produced ovine BMP15 or E.coli-produced ovine GDF9) was incubated with the primary antibody at a ratio of either $25: 1,3: 1$ or $10: 1$ by mass (indicated by relevant figures) in $200 \mu \mathrm{L}$ of membrane wash buffer in a $1.5 \mathrm{~mL}$ microcentrifuge tube. The blocking-peptide to antibody ratio by mass stated for each figure (stated as $\mathrm{w} / \mathrm{w}$ ) represents the ratio between the total mass of the blocking peptide and the total mass of the antibody present. The tubes were placed on a Vibrax VXR Basic vibrating stirrer (IKA) set to low speed and incubated 24 hours at $4^{\circ} \mathrm{C}$. Following incubation, the tubes were centrifuged at $600 \mathrm{~g}$ for 5 minutes on a Minispin Plus centrifuge (Eppendorf) and the supernatant was added to the membrane in a final volume of $6 \mu \mathrm{L}$ of blocking buffer. Immunodetection and chemiluminescent detection of these blots has been previously described (see Sections 2.1.3.4 and 2.1.3.5, respectively). In experiments where a preadsorbed antibody was to be compared to a non-preadsorbed antibody, the nonpreadsorbed antibody was subject to the same conditions as the preasdorbed antibody. 


\subsubsection{Calculation of band size}

The molecular weights of the bands were determined by first calculating the $R_{f}$ of each band using the protein ladder as guidance (where $R_{f}=$ the distance migrated of the protein / the distance migrated by the dye front for the resolving portion of each gel). The $R_{f}$ values were then plotted on the $x$ axis against the $\log _{10}$ (molecular weight) of each band on the $y$ axis. A linear regression was fitted to the points and the formula of the resultant regression line was calculated. The $R_{f}$ values of the chemiluminescent labelled proteins was then measured and the formula of the linear regression line was used to convert the $R_{f}$ values to their respective molecular weight values for each band. When a band size varied slightly across multiple blots, an approximate range for the band size is stated.

\subsubsection{Densitometric analysis}

Semi-quantitative analysis of the relative density of bands was performed using ImageJ software (version 1.50b with Java 1.8.0_60 64-bit; National Institutes of Health (NIH); Schneider et al., 2012). Raw TIFF format 16 bit images of the bands taken by the Omega Lum G imaging system (Aplegen) (see Section 2.1.3.5) were transferred to a PC for analysis. The images were first checked for saturation using Image Studio Lite version 5.2 (Li-COR Biosciences) and only images taken at exposures where no saturation was detected in all bands were used for analysis. The image to be quantified was loaded into Image J software. The mean grey value was set as the only measurement category, and image type was set to 16 bit. The rectangle selection tool was used to create a frame around the largest band to be analysed and this rectangular selection frame was saved and subsequently used for all bands analysed. The rectangular selection frame was placed over each band to be analysed, and the mean grey value was calculated. The mean grey value was represented by a value between 0 and 65,536 due to the 65,536 possible shades of grey for each pixel in a 16 bit image. The higher the number, the greater the density of the band, with a value of 65,536 representing a selection box which was filled completely with black pixels, and any lower numbers representing the mean of the shade value of the pixels within the selection box. Within each blot, the selection frame was moved to three separate points 
where there were no bands and the mean grey value was calculated. The average of these three values was deemed to be the background, and this was subtracted from the mean grey values of each band on that blot to give the final density value for each band which was used for future analysis.

\subsubsection{Graphs and Statistical Analysis}

Densitometry data was graphed and analysed using Prism Software (version 6.01; Graphpad). Density values were presented either relative to $\beta$-actin, or to the number of oocytes present in each sample. Linear regressions were fitted to densitometry data, and statistical analysis of the slopes was performed using Prism Software Version 6.01 (Graphpad) where applicable.

When the values were presented relative to $\beta$-actin, the density of the BMP15 bands were measured, and then the blots were stripped and relabelled with an anti- $\beta$-actin antibody. The BMP15 values for a given blot were presented relative to the density of the $\beta$-actin band found in the oocyte lysate of the same blot. As $\beta$-actin was only present in the oocyte lysate sample of each blot, the BMP15 band density values measured in the oocyte conditioned media was presented relative to $1.25 \mathrm{X}$ the density of the $\beta$-actin band found in the oocyte lysate of the same blot. A multiple of 1.25 was used to adjust for the fact that $25 \%$ more oocytes were used in the oocyte conditioned media samples.

When the values were presented relative to the number of oocytes, the density of the BMP15 bands in the oocyte samples were adjusted by dividing the band density values by a factor determined by the number of oocytes in the sample/100. For example, in rat oocyte lysate samples where 200 oocytes were used, the density values were divided by 2 $(200 / 100=2)$. These oocyte-count adjusted values were then normalised against the recombinant pig mature BMP15 band in the positive control to account for inter-blot variation. To do this, the adjusted density value for a band on a given blot was divided by the density of the mature band in the recombinant HEK-293 produced pig BMP15 positive control on the same blot. 
When comparisons were made within a given species, such as between the promature and mature BMP15 band densities, or between the promature:mature ratios of the oocyte lysates and oocyte conditioned media samples, a Student's $t$-test was performed to determine if there was a significant difference between the groups. When comparisons were made across all four species, a one-way ANOVA with Bonferroni's post-hoc test was performed. In all comparisons, $\mathrm{P} \leq 0.05$ was considered significant. When band density values were presented relative to the density of another band, such as when the BMP15 band densities were presented relative to the $\beta$-actin band densities, the ratios were calculated within each replicate first, and then the mean of the ratios from the three replicates was calculated. 


\section{Chapter 3: Results}

\subsection{The monoclonal antibody Mab61B has a significantly reduced ability to detect promature BMP15, and a moderately reduced ability to detect mature BMP15 on a Western blot, compared to Mab61A}

The first aim of this study was to characterise a novel monoclonal antibody provided inhouse (Mab61B) that had been generated against a 14 amino acid sequence at the $\mathrm{N}$ terminus of mature ovine BMP15, and compare it to a previously characterised antibody (Mab61A; Lin et al., 2012) (see Appendix II for details on the antibodies). Mab61B was first used to detect recombinant sheep, pig and deer BMP15 on a Western blot under the originally optimal conditions for Mab61A (Figure 3.1 A). Under these conditions, Mab61B showed a moderate ability to pick up mature BMP15 from all three species (band between 11-14 kDa); however it lacked the ability to detect promature BMP15. Instead, two faint bands were detected in the $\sim 55-70 \mathrm{kDa}$ range which were also detected in the negative controls. Under the same conditions, Mab61A showed a good sensitivity for both the BMP15 mature ( 11-14 kDa band) and promature BMP15 ( 47-50 kDa band) protein in each species tested, with a particularly strong pig BMP15 promature band detected (Figure 3.1 C). Mab61A also detected two distinct unknown bands in the $~ 60-75 \mathrm{kDa}$ range which were also found in the negative controls.

To determine whether the lack of promature BMP15 detection with Mab61B was due to a lack of sensitivity caused by the Western blotting protocol being used, a range of optimisation tests were undertaken (Figure 3.1 E). Under the optimised conditions, Mab61B had an increased ability to detect the mature BMP15 protein, which was coupled with a large increase in the density of the two non-specific bands detected in the $~ 55-70$ kDa range, and still no detection of the promature BMP15 protein (Figure 3.1 B), despite Mab61A demonstrating that it was present in the samples (Figure 3.1 C). To test whether the detection of promature BMP15 with Mab61B was dependent on the amount of protein loaded on the gel, a titration of recombinant pig BMP15 conditioned media was performed and protein detection was measured using Mab61B under optimal conditions (Figure 3.1 
D). Even when an excessive amount ( $8 \mu \mathrm{L})$ of the pig BMP15 sample was loaded onto the gel, there were no obvious bands detected at the expected promature BMP15 size.

The following experiment tested whether Mab61B could detect the native promature and mature forms of BMP15 in oocyte lysate samples (Figure 3.2 A). The antibody was able to detect mature BMP15 in the oocyte lysate samples from sheep, pigs, deer and rats as indicated by the band at $11-14 \mathrm{kDa}$ in each species. Once again, there was no distinct promature band detected in any of the species tested, suggesting that Mab61B is unable to detect the promature BMP15 protein of any of the species tested in the context of a Western blot. The antibody Mab61B also detected a range of non-specific proteins between $\sim 15$ and $50 \mathrm{kDa}$, and a distinct protein at $\sim 58 \mathrm{kDa}$ in size, which was a similar size to one of the non-specific bands observed in both the positive and negative controls. The blot depicted in Figure 3.2 A was then stripped of the Mab61B antibody, and reprobed with the Mab61A antibody to determine if promature BMP15 was present in the oocyte lysate samples, and to compare the sensitivity of Mab61A with Mab61B (Figure $3.2 \mathrm{~B}$ ). The Mab61A antibody was able to detect mature BMP15 as a distinct band in the $11-14 \mathrm{kDa}$ range in the sheep, pig and deer oocyte lysate samples, but not in the rat oocyte lysate samples. The lack of a band at the known size of mature BMP15 in the rat oocyte lysate sample was attributed to a small amount of protein being further lost in the stripping process. The unavoidable loss of some protein during membrane stripping was established in earlier optimisation experiments of the stripping procedure (data not shown). In later experiments, it was determined that the Mab61A antibody detected mature rat BMP15 with a similar affinity to the Mab61B antibody (data not shown). Unlike Mab61B, Mab61A was able to detect a large amount of promature BMP15 in sheep, pig and deer oocyte lysate samples, and a small amount in the rat samples as indicated by a distinct band in the $\sim 47-$ $50 \mathrm{kDa}$ range for each species (Figure 3.2 B). There were also several bands detected in the sheep, pig and deer oocyte lysate samples that were $\sim 35-45 \mathrm{kDa}$ in size, and did not fit the known size of the mature or promature proteins. 

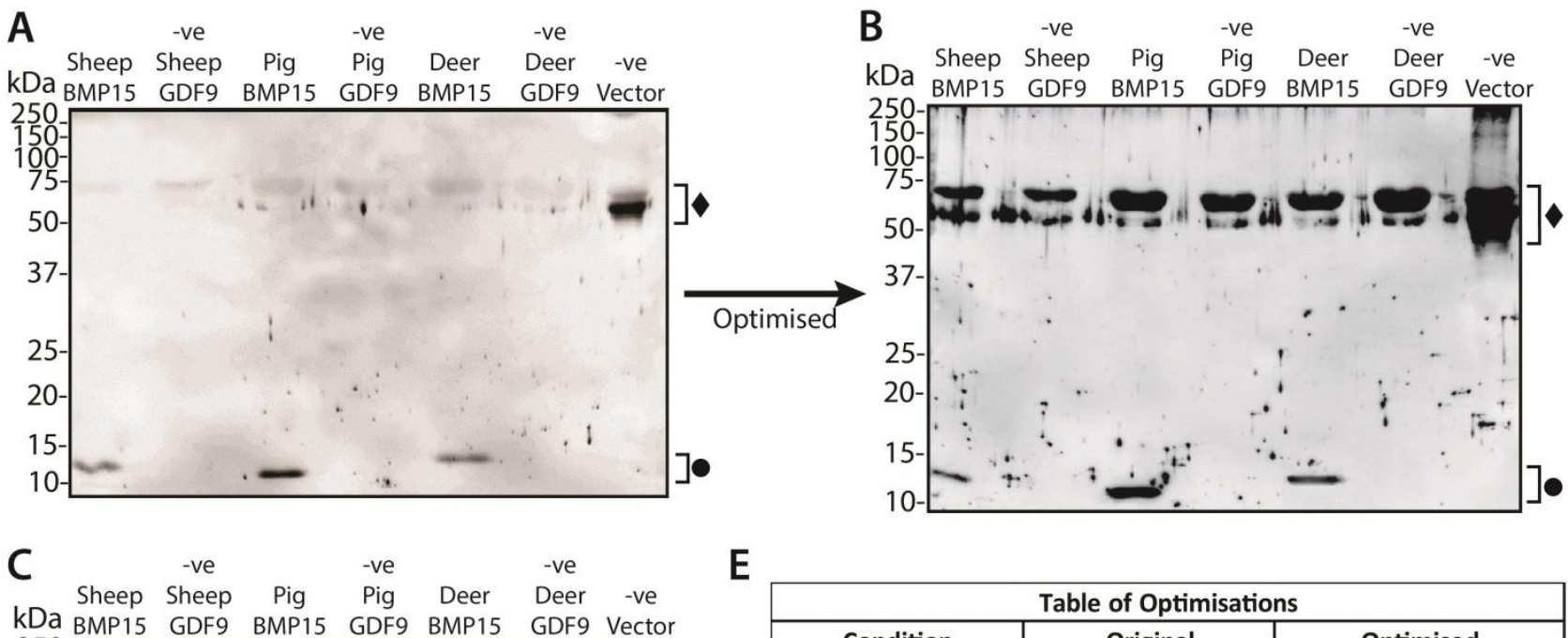

E
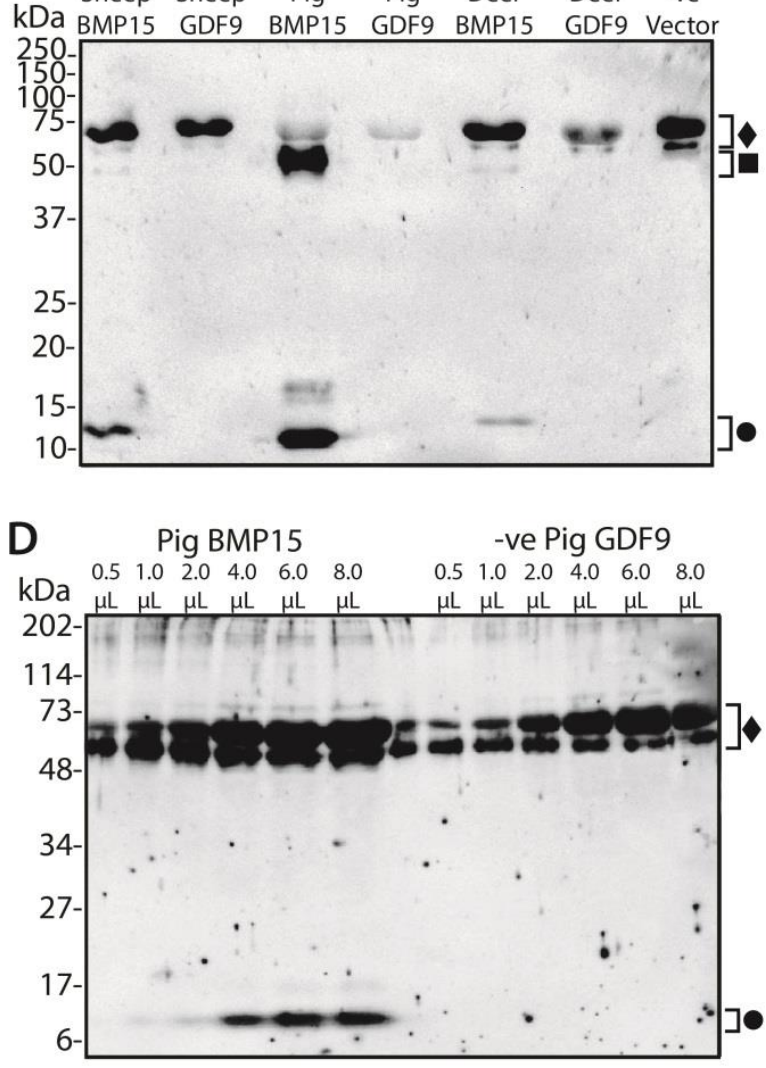

\begin{tabular}{|c|c|c|}
\hline \multicolumn{3}{|c|}{ Table of Optimisations } \\
\hline Condition & Original & Optimised \\
\hline $\begin{array}{l}\text { Membrane blocking } \\
\text { solution }\end{array}$ & $\begin{array}{l}5 \% \text { Skim milk powder } \\
\text { dissolved in High-Q water. } \\
\text { No Centrifugation } \\
\text { No Filtration }\end{array}$ & $\begin{array}{l}2 \% \text { Casein dissolved in High-Q } \\
\text { water. } \\
\text { Centrifuged at } 10000 \mathrm{~g} \text { for } 1 \\
\text { hour. } \\
\text { Supernatant filtered through } \\
0.45 \mu \mathrm{m} \text { Syringe filter }\end{array}$ \\
\hline Blocking Time & 1 hour & 1 hour \\
\hline $\begin{array}{l}\text { Blocking buffer added } \\
\text { with the antibodies }\end{array}$ & $\begin{array}{l}5 \% \text { Skim milk powder } \\
\text { dissolved in High-Q water. } \\
\text { No Centrifugation } \\
\text { No Filtration }\end{array}$ & $\begin{array}{l}1 \% \text { Casein dissolved in High-Q } \\
\text { water. Centrifuged at } 10000 \mathrm{~g} \\
\text { for } 1 \text { hour. } \\
\text { Supernatant filtered through } \\
0.45 \mu \mathrm{m} \text { Syringe filter }\end{array}$ \\
\hline Primary antibody & $1 \mu \mathrm{g} / \mathrm{ml}$ in blocking buffer. & $2 \mu \mathrm{g} / \mathrm{ml}$ in blocking buffer. \\
\hline $\begin{array}{l}\text { Primary antibody } \\
\text { incubation time and } \\
\text { temperature }\end{array}$ & $\begin{array}{l}3 \text { hours at room } \\
\text { temperature on see-saw } \\
\text { rocker }\end{array}$ & $\begin{array}{l}12 \text { hours at } 4^{\circ} \mathrm{C} \text { on see-saw } \\
\text { rocker }\end{array}$ \\
\hline $\begin{array}{l}\text { Secondary Antibody } \\
\text { (Peroxidase-conjugated } \\
\text { Affinipure Rabbit Anti- } \\
\text { mouse IgG) }\end{array}$ & $\begin{array}{l}0.2 \mu \mathrm{g} / \mathrm{ml} \text { in blocking } \\
\text { buffer. }\end{array}$ & $0.4 \mu \mathrm{g} / \mathrm{ml}$ in blocking buffer. \\
\hline $\begin{array}{l}\text { Secondary antibody } \\
\text { incubation time and } \\
\text { temperature }\end{array}$ & $\begin{array}{l}1 \text { hour at room } \\
\text { temperature on see-saw } \\
\text { rocker }\end{array}$ & $\begin{array}{l}6 \text { hours at room temperature } \\
\text { on see-saw rocker }\end{array}$ \\
\hline $\begin{array}{l}\text { Sample heating time and } \\
\text { temperature (after } \\
\text { addition of loading buffer } \\
\text { prior to loading on gel) }\end{array}$ & $95^{\circ} \mathrm{C} 5 \mathrm{~min}$ & $\begin{array}{l}95^{\circ} \mathrm{C} 5 \mathrm{~min} \text { (no change across } \\
\text { a range of heating } \\
\text { temperatures and times) }\end{array}$ \\
\hline $\begin{array}{l}\text { Reducing Agent in } \\
\text { reducing } 2 X \text { loading buffer }\end{array}$ & $10 \% 2 \mathrm{ME}$ & $10 \% 2 \mathrm{ME}+35 \mathrm{mM}$ DTT \\
\hline
\end{tabular}

Figure 3.1: Mab61A showed a low affinity for recombinant promature BMP15 compared to Mab61A, even following an optimised Western blot protocol. (A) Western blot labelled with BMP15 antibody Mab61B using original Western blot conditions (conditions listed under "Original" in (E)) which were previously optimised in-house for the Mab61A antibody. The $\mathbf{1}^{\text {st }}, 3^{\text {rd }}$ and $5^{\text {th }}$ lanes depict $4 \mu \mathrm{L}$ of recombinant HEK-293-produced sheep, pig and deer BMP15 conditioned media, respectively. The $2^{\text {nd }}$, $3^{\text {rd }}$ and $6^{\text {th }}$ lanes depict $4 \mu \mathrm{L}$ of recombinant HEK-293 produced sheep, pig and deer GDF9 conditioned media, respectively (negative controls). Lane 7 depicts $4 \mu$ L of untransfected HEK-293 conditioned media (negative control). (B) Western blot of BMP15 labelled with Mab61B with same lanes as (A) performed under conditions optimised for Mab61B as outlined in the table of optimisations (E). (C) Western blot under same conditions as (A) except labelled with the Mab61A antibody. (D) Titrations of recombinant HEK-293-produced pig BMP15 and GDF9 conditioned media on a Western blot performed under optimised conditions as indicated by the table of optimisations (E) and labelled with Mab61B BMP15 antibody. (E) Table of the different optimisation procedures tested for Western blotting. All blots were performed under reducing conditions. 


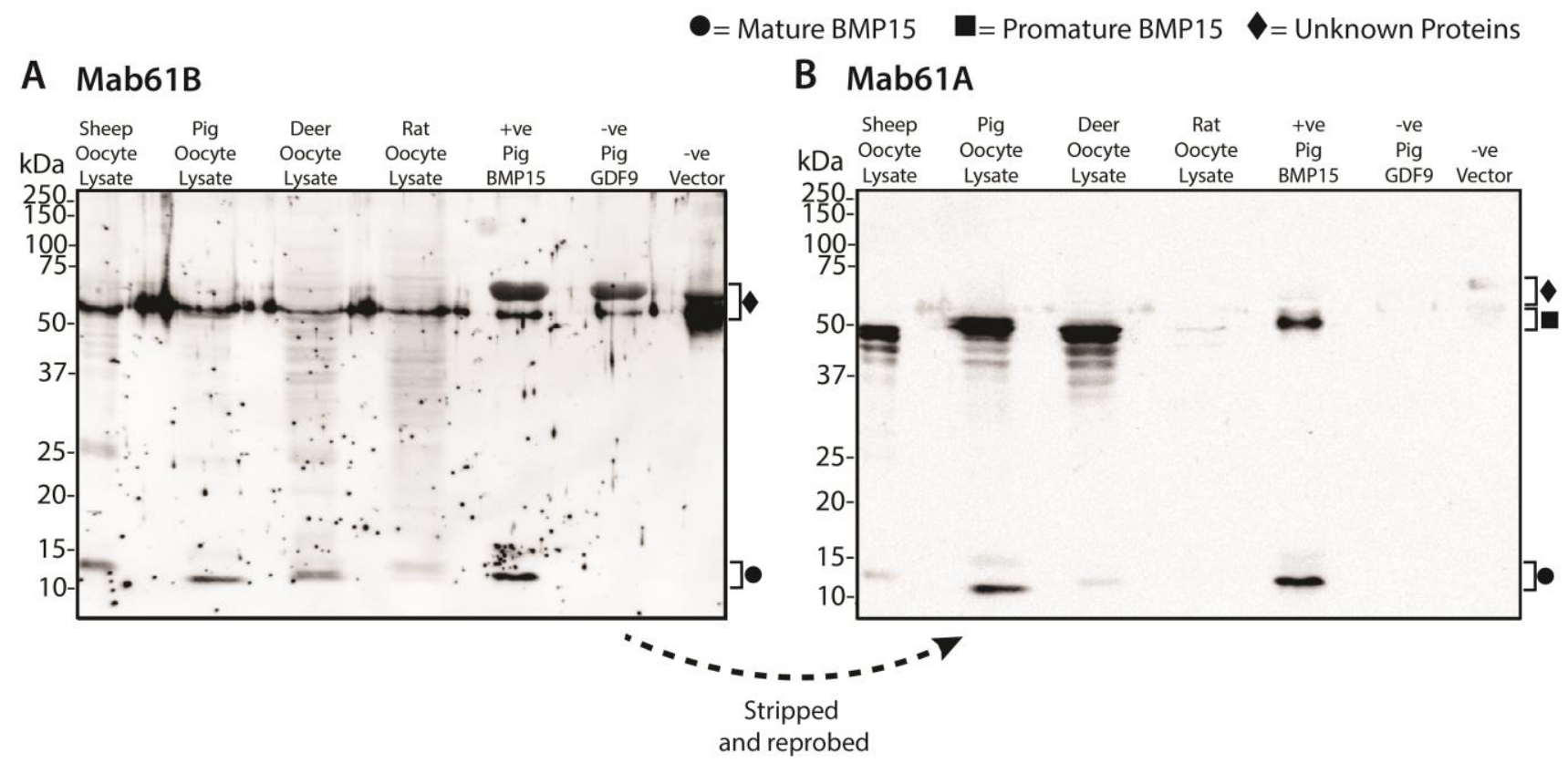

Figure 3.2: Mab61B shows no affinity for the promature BMP15 protein in oocyte lysates, as compared to Mab61A. (A) Western blot labelled with Mab61B antibody. Lanes 1-3 were loaded with the lysate of 100 sheep, pig and deer oocytes, respectively, and Lane 4 was loaded with the lysate of 200 rat oocytes. Lane 5 contained $4 \mu \mathrm{L}$ of recombinant HEK-293-produced pig BMP15 conditioned media (positive control). Lanes 6 and 7 were negative controls containing $4 \mu \mathrm{L}$ of recombinant HEK-293-produced pig GDF9 conditioned media, and $4 \mu \mathrm{L}$ of untransfected HEK-293 conditioned media, respectively. (B) The blot from (A) was stripped and reprobed with Mab61A (B). All blots were performed under reducing conditions.

\subsection{The monoclonal antibodies Mab61B and Mab61A have different non-specific binding targets, and different levels of specificity for their BMP15 target}

To determine the specificity of the bands detected by both the Mab61B and Mab61A BMP15 antibodies, a range of preabsorption experiments with different antigens were performed (Figure 3.3). Firstly, the peptide used to generate Mab61B in mice (Peptide B; see Appendix II) was used. Preabsorption of the Mab61B antibody with 25x w/w Peptide B lead to the loss of the band detected at $\sim 11 \mathrm{kDa}$ (mature BMP15) in recombinant HEK-293produced pig BMP15. The unknown bands in the $\sim 55-70 \mathrm{kDa}$ size range were still detected with a similar affinity to those observed when using the non-preabsorbed Mab61B antibody (Figure 3.3 A). This suggests that the unknown bands in the $\sim 55-70 \mathrm{kDa}$ range were most likely due to high-affinity non-specific binding. The blot was then stripped and 
re-probed with Mab61B antibody that had been preabsorbed with 10x (w/w) E.coli produced ovine GDF9 to test for cross reactivity with the closely related protein (Figure 3.3 A). A band at the size of the mature BMP15 protein could still be detected when Mab61B was preabsorbed with GDF9, albeit at a slightly reduced density. This indicates that Mab61B exhibits minimal affinity for GDF9.

In a separate experiment, Mab61B was preabsorbed with 10x (w/w) E.coli-produced ovine BMP15 (Figure $3.3 \mathrm{C}$ ). In this case, the mature band at $\sim 11 \mathrm{kDa}$ disappeared, as did the $55 \mathrm{kDa}$ band that represented one of the two unknown bands, however the higher molecular weight band at $70 \mathrm{kDa}$ remained. Just like with the peptide B preabsorption, the presence of this band following preabsorption with BMP15 suggests that it may be due to high affinity non-specific binding of the antibody. The absence of the $\sim 55 \mathrm{kDa}$ band suggests that this band is either due to specific binding of the antibody, or non-specific binding of the antibody to a protein that has significantly lower affinity than for the BMP15 used for preabsorption. The disappearance of the $\sim 11 \mathrm{kDa}$ band again supports the idea that this band represents mature BMP15 protein.

The same experiments from Figures 3.3 $\mathrm{A}$ and $\mathrm{C}$ were repeated using the Mab61A antibody (Figures 3.3 B and 3.3 D). The Mab61A antibody was partially preabsorbed by peptide B, however it was still able to detect both promature BMP15 ( 50 kDa band) and mature BMP15 ( 11-14 kDa) (Figure 3.3 B). It is important to note that Peptide B contains only 8 of the 15 amino acids which Mab61A is known to target (see Appendix II), thus only partial absorption was expected. These results suggest that for effective binding, Mab61A relies on the presence of the remaining 7 amino acids of its known target region, which are not part of the peptide B sequence. The blot was then stripped and then re-probed with Mab61A which had been preabsorbed with E.coli-produced ovine GDF9 to test for cross reactivity (Figure $3.3 \mathrm{~B}$ ). In this case the results were nearly identical to those seen using Mab61B in Figure 3.3 A, which suggests that Mab61A has minimal affinity to GDF9. The preabsorption of the Mab61A antibody with E.coli produced ovine BMP15 led to a complete loss of all bands on the blot, including the $\sim 60-75 \mathrm{kDa}$ bands of unknown protein (Figure $3.3 \mathrm{D}$ ). This suggests that either the antibody is binding to all bands on the blot specifically, or that the antibody is at least binding to the E.coli-produced ovine BMP15 with a higher affinity than other non-target proteins. 
Overall, these results substantiate the general dogma that the bands at $\sim 11 \mathrm{kDa}$ and $\sim 50$ $\mathrm{kDa}$ represent the mature and promature forms of BMP15, respectively. The bands in the 60-75 kDa size range were present in both the positive and negative controls and as such, are likely due to non-specific binding.

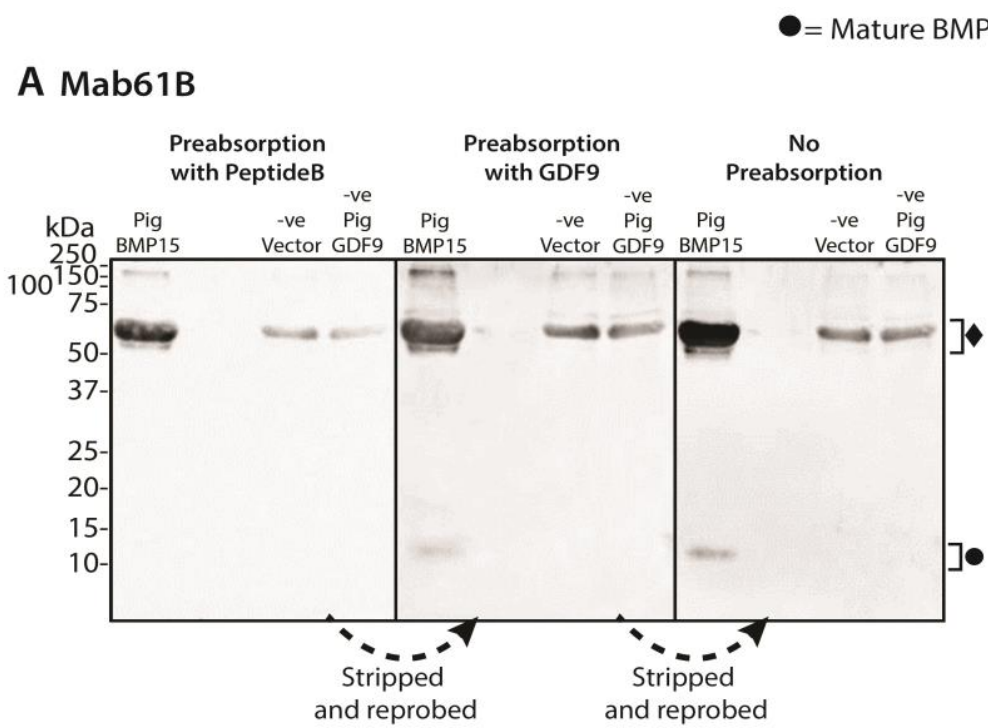

$\boldsymbol{\square}=$ Promature BMP15 $\boldsymbol{\nabla}=$ Unknown Proteins

C Mab61B

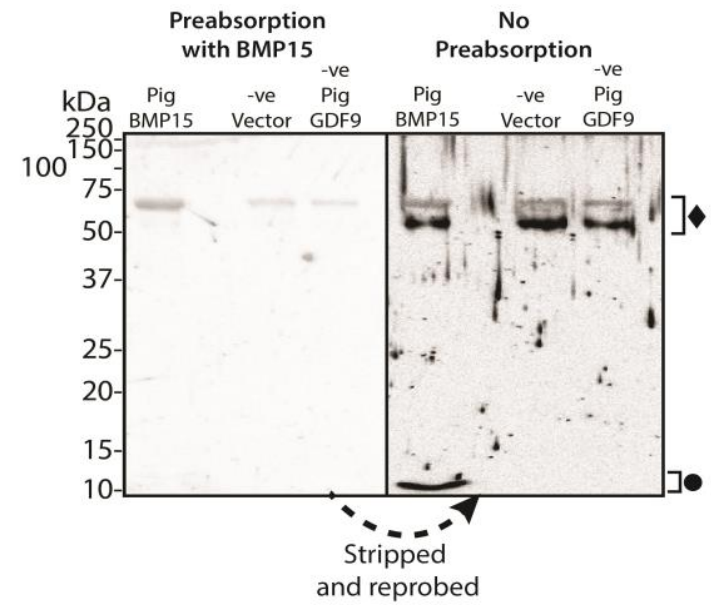

B Mab61A

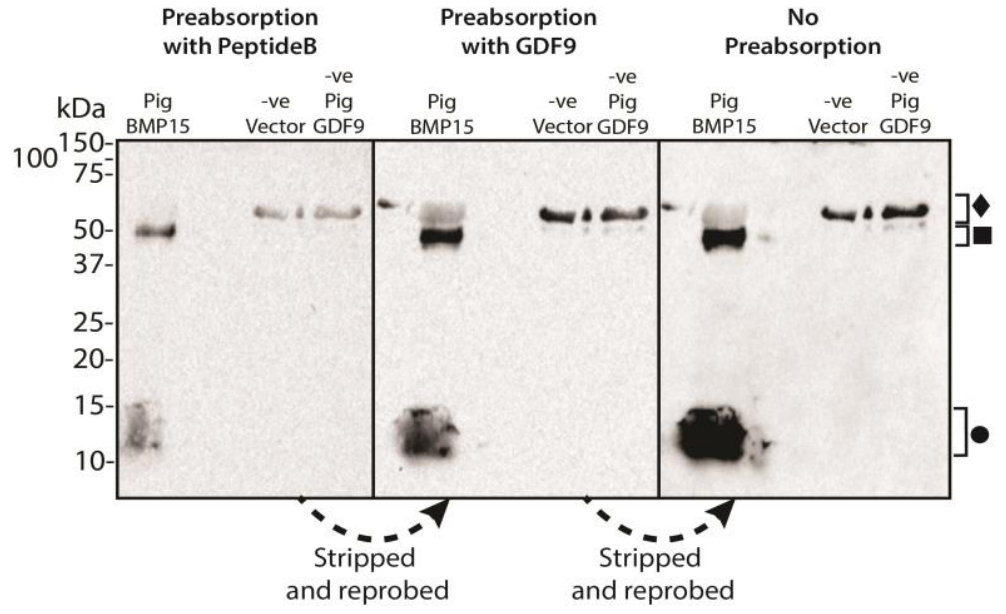

D Mab61A

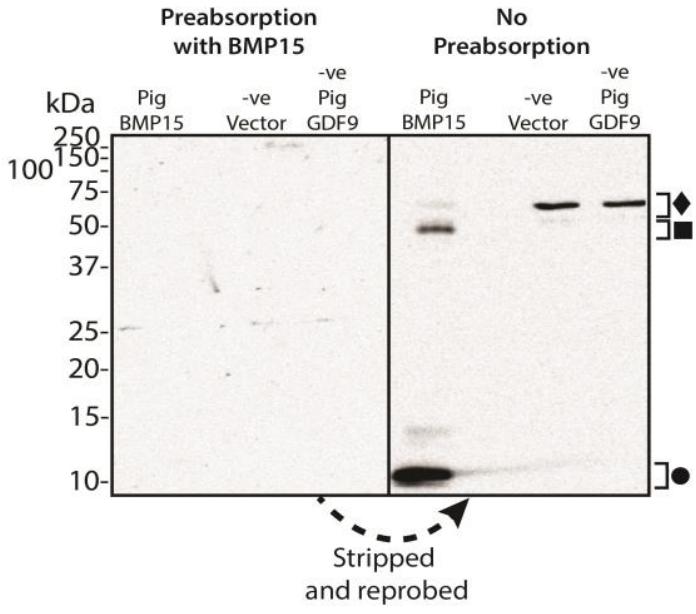

Figure 3.3: Preabsorption of Mab61B and Mab61A antibodies differ with antigen, and exhibit divergent non-specific binding on Western blots. (A) Mab61B was preabsorbed with 25x w/w Peptide B. This blot was then stripped and reprobed with Mab61B preabsorbed with 10x w/w E.coli-produced ovine GDF9. The blot was then stripped a second time and re-probed with Mab61B without preabsorption. Lane 1 contains $4 \mu \mathrm{L}$ of recombinant HEK-293-produced pig BMP15 conditioned media (positive control). Lanes 2 and 3 are negative controls containing $4 \mu \mathrm{L}$ of untransfected HEK-293 conditioned media, and $4 \mu \mathrm{L}$ of recombinant HEK-293-produced pig GDF9 conditioned media, respectively. (B) The same Western blot layout in (A) was performed with Mab61A. (C) A Western blot with the same layout as (A) and (B) was probed with Mab61B which had been preabsorbed with $10 \mathrm{x}$ w/w E.coli-produced ovine BMP15. The blot was then stripped and re-probed with Mab61B without preabsorption. (D) The same set of experiments as described in (C) was performed with Mab61A. All blots were performed under reducing conditions. 


\subsection{High affinity non-specific bands in close proximity to specific target bands on Western blots may partially block the primary antibody from binding its specific target}

The Western blots immuno-labelled with Mab61B antibody in Figures 3.1, 3.2 and 3.3 demonstrated that the antibody binds unknown non-specific bands in the $~ 55-75 \mathrm{kDa}$ region with high affinity. These unknown bands were in close proximity to the target BMP15 promature band, unlike the unknown bands observed when using Mab61A (Figure 3.1 $\mathrm{B}$ and $\mathrm{C}$ ). To test whether the close proximity of the high affinity unknown proteins were blocking or masking the ability of Mab61B to bind its promature BMP15 target, a 7.5\% acrylamide gel was used in place of a $13.5 \%$ acrylamide gel in order to provide a greater separation between the $\sim 55-75 \mathrm{kDa}$ bands, and the target BMP15 promature band at $\sim 50$ kDa (Figure 3.4). For reference, Figure 3.4 A and B depicts the same results from the $13.5 \%$ gel in Figure 3.2 with just the region of interest displayed. The same region of interest from a 7.5\% acrylamide gel is displayed in Figure 3.4 C and D. In this blot labelled with Mab61B (Figure $3.4 \mathrm{C}$ ), distinct bands appear in the $\sim 48-50 \mathrm{kDa}$ region which were not observed in the $13.5 \%$ gel (Figure 3.4A). These bands appear to correspond to the BMP15 promature bands observed when using the Mab61A antibody on the same blots after stripping (Figures 3.4 $B$ and $D$ ). This suggests that the unknown non-specific bands in the $\sim 55-75 \mathrm{kDa}$ region may have blocked Mab61B from binding to its promature BMP15 target in blots shown previously. It should be noted that with the exception of the rat, the BMP15 promature bands detected using Mab61B were much fainter than those detected with Mab61A after stripping, suggesting that the Mab61B antibody has a lower affinity for this protein. 


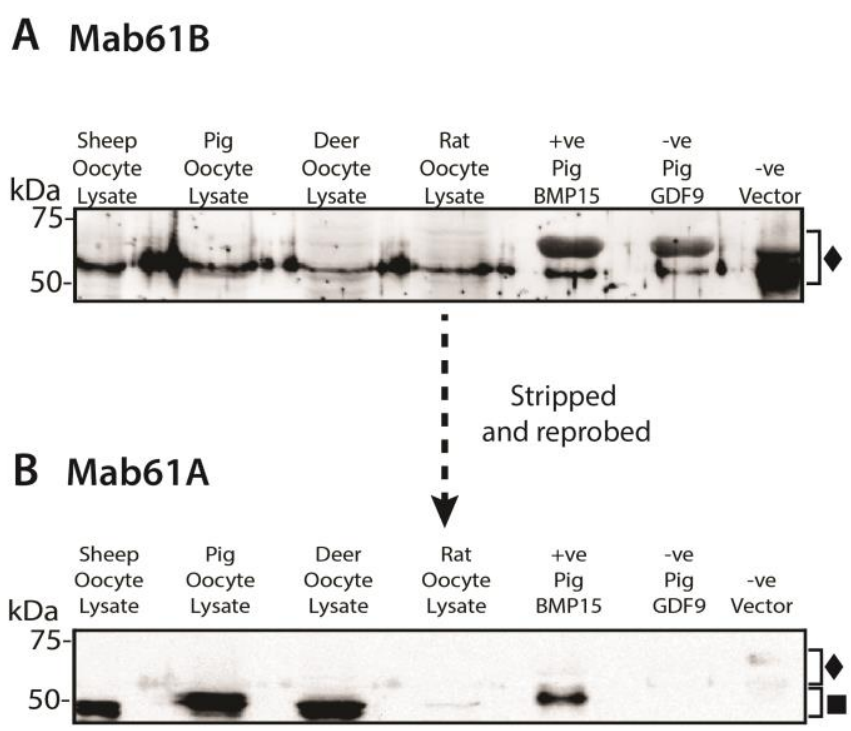

\section{Mab61B}

Promature BMP15 = Unknown Proteins

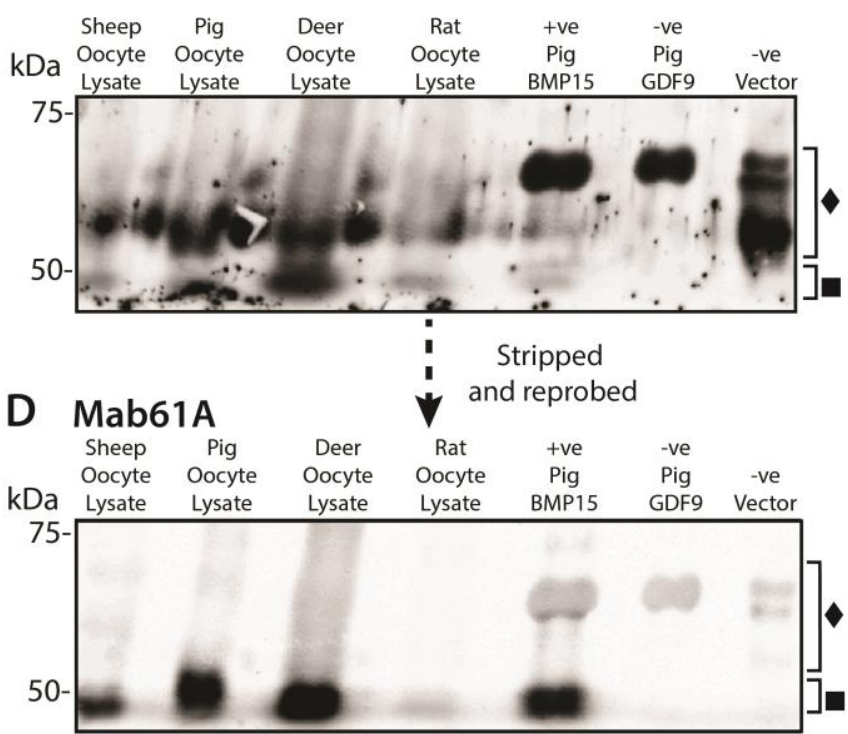

Figure 3.4: Mab61B can bind promature BMP15 on Western blots when it is separated from non-target proteins of a similar size. (A) and (B) For reference, portions of Western blots made using $13.5 \%$ acrylamide gels from Figure 3.2 are shown. The blot in $(A)$ was probed with Mab61B before being stripped and re-probed with Mab61A, shown in (B). The first 4 lanes are loaded with oocyte lysate from 100 sheep, pig and deer oocytes, and 200 rat oocytes, respectively. Lane 5 was loaded with $4 \mu \mathrm{L}$ of recombinant HEK-293-produced pig BMP15 conditioned media (positive control), and Lanes 6 and 7 were loaded with $4 \mu \mathrm{L}$ of recombinant HEK-293-produced pig GDF9 conditioned media, and $4 \mu \mathrm{L}$ of untransfected HEK-293 conditioned media, respectively (negative controls). (C) A portion of a blot with an identical lane structure to $(\mathrm{A})$ made using a 7.5\% acrylamide gel. The blot was labelled with Mab61B. (D) The blot in (C) was stripped and re-probed with Mab61A. All blots were performed under reducing conditions. 


\subsection{The optimal numbers of oocytes for detection of intra- oocyte molecular forms of BMP15 in rats, sheep, pigs and deer are 200, 100, 80 and 100, respectively}

Due to the Mab61A antibody exhibiting a higher affinity and greater sensitivity towards BMP15, and also its ability to detect both mature and promature forms (as demonstrated in Figures 3.1-3.4), the Mab61A antibody was used for all future experiments.

To determine the optimal number of oocytes required for detection of the molecular forms of BMP15 for each species, titrations of lysed oocytes were performed. For the rat, sheep, deer and pig, 200, 100, 100 and 50 lysed oocytes were sufficient for detecting both the promature ( $\sim 50 \mathrm{kDa})$ and mature $(\sim 14-16 \mathrm{kDa})$ forms of BMP15 (Figure 3.5). However, in later experiments (data not shown), it was revealed that detection of the mature BMP15 band was inconsistent when using only 50 lysed pig oocytes, and that 100 oocytes may have led to band saturation. Thus, an intermediate number of 80 lysed oocytes were used.

Titrations were not performed to determine the number of oocytes to be used in the oocyte conditioned media samples. Instead, it was decided that the number of oocytes incubated per $25 \mu \mathrm{L}$ conditioned media sample would be $25 \%$ more than the number of oocytes used in the oocyte lysate samples. This $25 \%$ increase in number was chosen due to the amount of BMP15 secreted into the media by the oocytes in an 18 hour period being much lower than that in lysed oocytes. The increase in the number used was limited to $25 \%$ because it was observed that an increase in oocyte number of $>25 \%$ caused a significant increase in the acidity of the media (as observed by colour change) during the 18 hour incubation period. This increased acidity may have affected the forms of the protein, and also compromised oocyte health. Given the higher levels of BMP15 present inside an oocyte compared to that secreted into the media, the rupture of a single unhealthy oocyte during incubation period could have significantly altered the results if its contents were released into the media. 


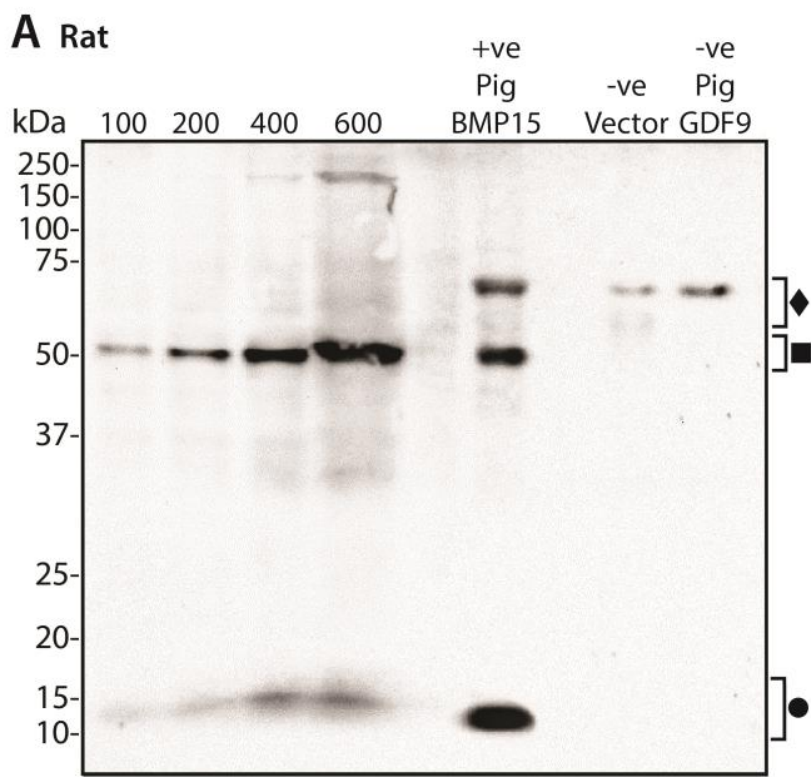

C Deer

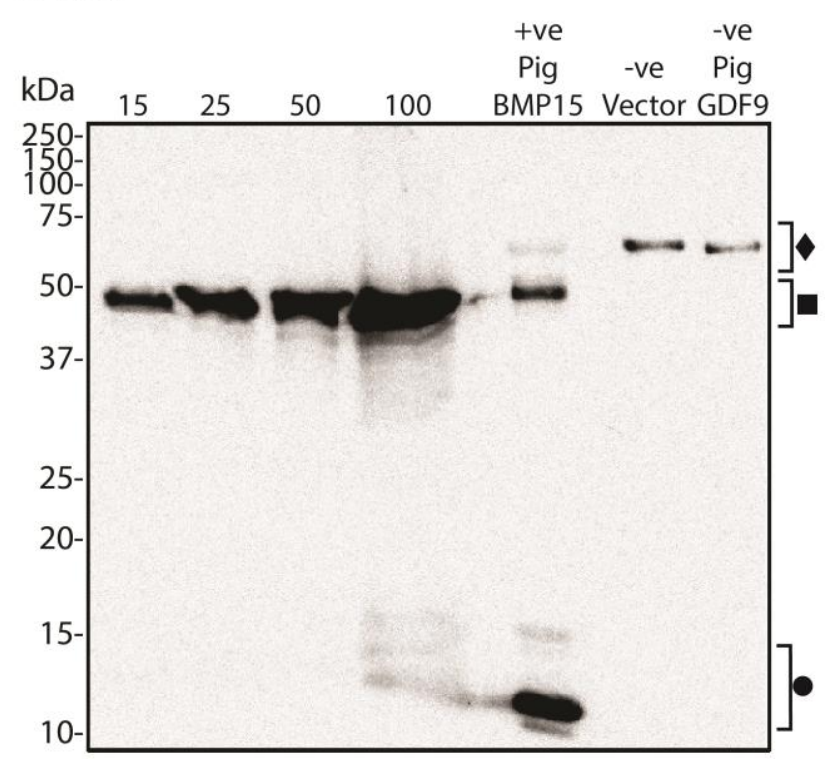

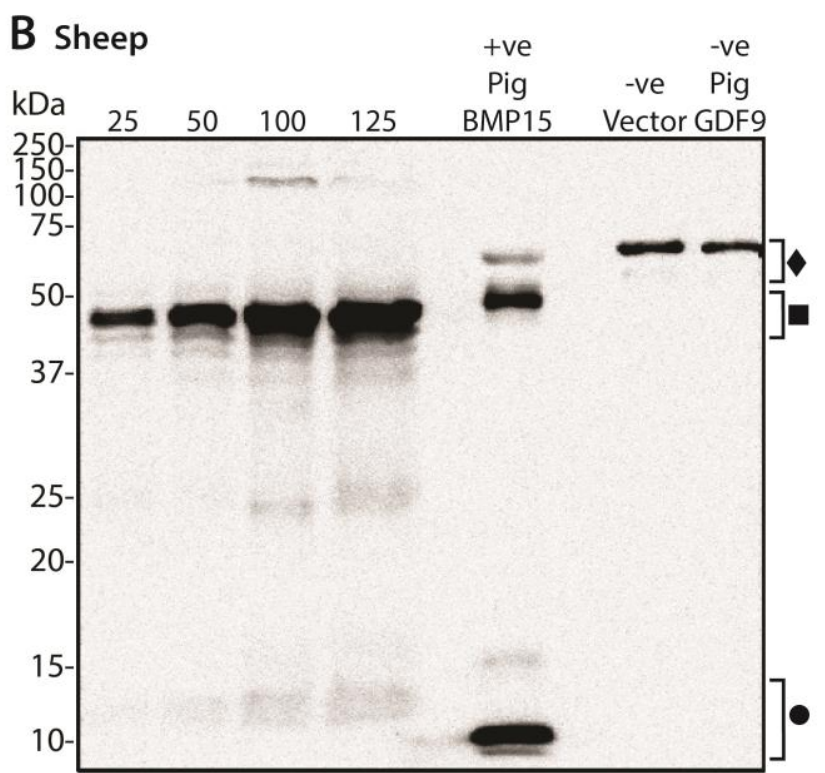

D Pig

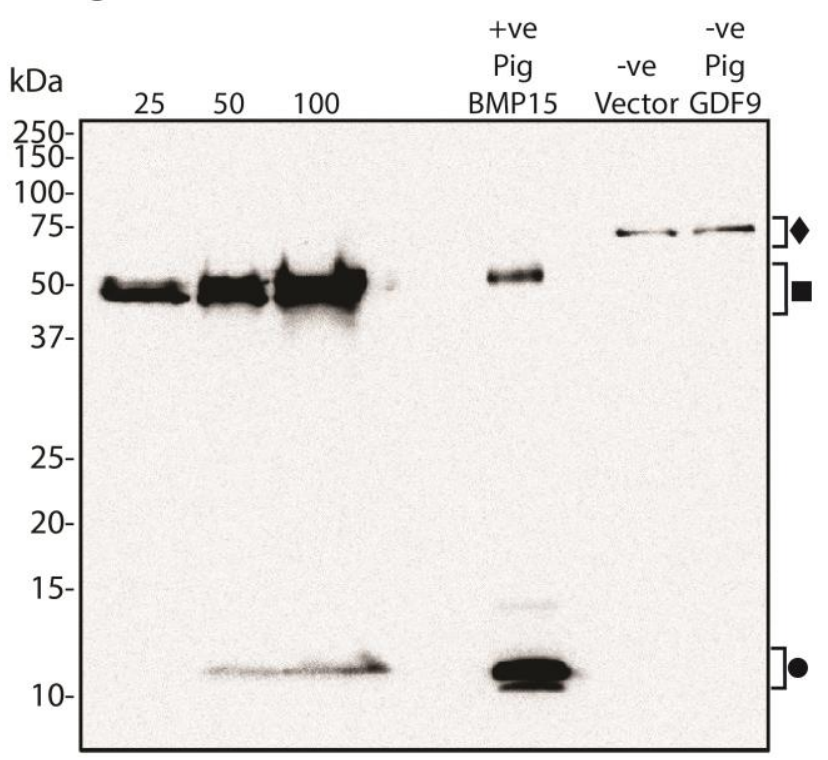

Figure 3.5: Titrations of rat, sheep, deer and pig lysed oocyte numbers for the detection of BMP15 using Mab61A. (A)(B)(C) Titrations of rat (A), sheep (B), and deer (C) oocyte lysates, respectively. The numbers above Lanes 1-4 represent the number of oocyte lysates used in that lane. Lane 5 contains $4 \mu \mathrm{L}$ of recombinant HEK-293-produced pig BMP15 conditioned media (positive control). Lanes 6 and 7 are negative controls containing $4 \mu \mathrm{L}$ of untransfected HEK-293 conditioned media and $4 \mu \mathrm{L}$ of recombinant HEK 293 produced pig GDF9 conditioned media, respectively. (D) Titrations of pig oocyte lysates. Lanes 1-3 represent the number of oocyte lysates used in that lane. Lanes 4-6 contain the various positive controls in the same order as seen in Lanes 5-7 in (A), (B) and (C). All blots were performed under reducing conditions. 


\subsection{Estimation of the concentration of mature BMP15 in the recombinant HEK-293 produced pig BMP15 conditioned media}

Three replicate Western blot experiments were performed under reducing conditions to quantify the concentration of mature BMP15 in the HEK-293 produced recombinant pig BMP15 conditioned media used throughout this study. The result of one of the three replicates is presented in Figure 3.6. The purpose of this experiment was to give a contextual frame of reference as to the approximate amount of protein present when viewing blots. The approximate concentration of the mature BMP15 protein was estimated by blotting a titration of the recombinant HEK-293 produced pig BMP15 conditioned media ( $\sim 12 \mathrm{kDa}$ ) alongside a titration of known quantities of $E$. coli produced mature ovine BMP15 ( 16-17 kDa) (Figure 3.6 A). The densities of these bands were plotted against the sample amount, and the values were fitted with a linear regression (Figures 3.6 B). The coefficients of determination $\left(R^{2}\right)$ for the conditioned media and E. coli produced BMP15 samples were at least 0.98 in all three replicates indicating a very strong linear correlation in both datasets. The mature BMP15 band that resulted from using $1 \mu \mathrm{L}$ of the HEK-293 conditioned media sample was not included in the linear correlation in any of the three replicates because its density fell outside the range detected in the $E$. coli produced samples. Each of the three replicates was graphed separately and the concentration of recombinant pig BMP15 in the HEK-293 conditioned media was estimated by comparing the densities of the three different HEK-293 conditioned media sample volumes with the standard curve generated using the $E$. coli produced BMP15 samples. The combined data from all three replicates estimated the concentration of recombinant pig BMP15 in the HEK-293 conditioned media to be $0.58 \mathrm{ng} / \mu \mathrm{L}$ (95\% Cl range of 0.55 to $0.61 \mathrm{ng} / \mu \mathrm{L}$ ). 


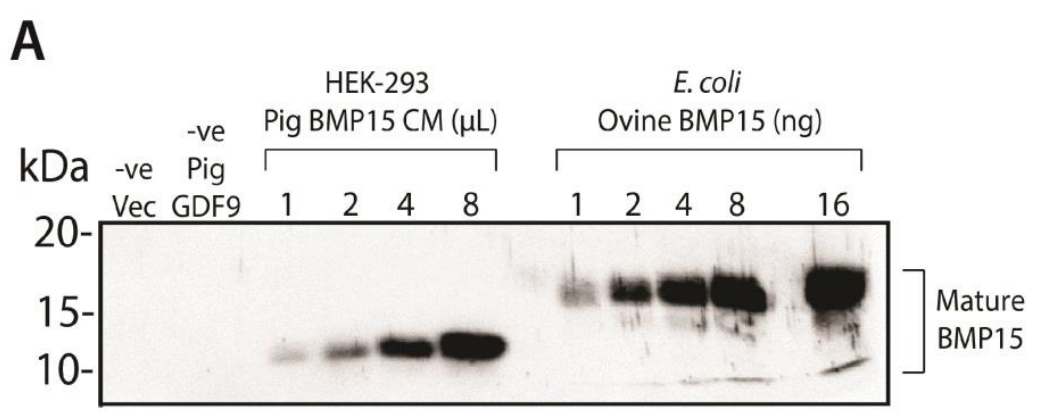

B

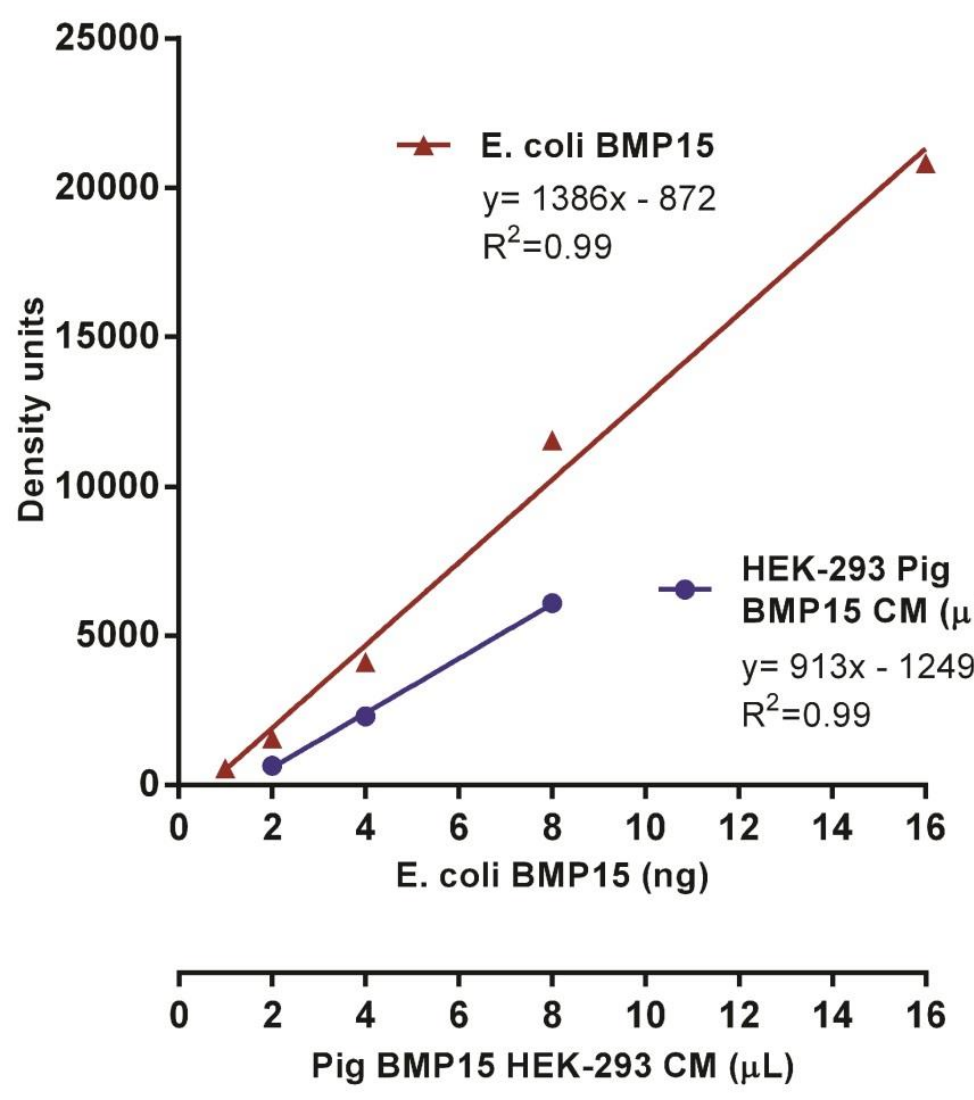

Figure 3.6: Estimation of the mature BMP15 concentration in the recombinant HEK-293 produced pig BMP15 conditioned media. (A) A Western blot performed under reducing conditions and labelled with Mab61A. Lanes 1 and 2 are negative controls containing $4 \mu \mathrm{L}$ of untransfected HEK-293 conditioned media and $4 \mu \mathrm{L}$ of recombinant HEK-293 produced pig GDF9 conditioned media, respectively. Lanes 3-6 represent titrations of recombinant HEK-293 produced pig BMP15 conditioned media, with the volume of media per lane indicated above each lane $(\mu \mathrm{L})$. Lanes 7-11 represent titrations of known quantities of E. coli produced ovine BMP15, with the amount of the protein present indicated above each lane (ng). There is a gap between Lanes 10 and 11 where no sample was loaded. (B) Scatterplot of the densities of the mature BMP15 bands found in Lanes 3-11 of (A). The data in red represents the densities of the E. coli produced mature ovine BMP15 bands, and relates to the top x-axis. The data in blue represents the densities of the recombinant HEK-293 produced pig BMP15 and relates to the bottom x-axis. The equations for each linear correlation, along with their $\mathrm{R}^{2}$ value are indicated below the relevant keys. The data in (A) and (B) represents one of three replicates. 


\subsection{The molecular forms of BMP15 in, and secreted from, oocytes of rats (litter size 7-16)}

To detect the molecular forms of BMP15 present in, and secreted from, the oocyte in different mammalian species, a series of Western blots were performed under nonreducing conditions, reducing conditions and finally conditions where the proteins were crosslinked before the addition of a reducing loading buffer. Representative Western blots under these conditions in which rat oocytes were used are displayed in Figure 3.7. A total of 200 rat oocytes were used per oocyte lysate lane and 250 rat oocytes were used to generate each sample of oocyte conditioned media.

Faint bands representing promature and mature BMP15 were observed in the rat oocyte lysates under all three conditions tested (Figure 3.7 A1, B1 and C1). Under reducing conditions, with or without crosslinking (Figures 3.7 B1 and C1), low levels of both promature (double band at $\sim 48-52 \mathrm{kDa}$ ) and mature BMP15 (band at $\sim 15-16 \mathrm{kDa}$ ) were detected. The molecular weights differed slightly from those observed in the positive control of recombinant pig BMP15, which is likely due to slight species differences in the proteins (c.f. Figure 3.9). Promature and mature BMP15 also appeared in the non-reduced oocyte lysate samples, and exhibited slightly higher molecular weights ( 55-58 kDa and $\sim 17 \mathrm{kDa}$, respectively) to those observed under reducing conditions. This may be due to existing intramolecular disulphide bonds remaining intact in the non-reducing buffer, hindering the passage of the protein through the gel.

In both the crosslinked samples under reducing conditions, and the non-reduced samples (Figures $3.7 \mathrm{C} 1$ and $\mathrm{A} 1$, respectively), there were bands representing unknown proteins in the $\sim 150-250 \mathrm{kDa}$ region of the oocyte lysates samples that were consistent across replicates. A band was observed in the same region in the reducing blot (without crosslinking), however this band was absent in the other two replicates (Figure 3.7 B1). When taken together, these bands indicate the presence of large covalently or possibly non-covalently bound protein complexes containing multimers of different molecular forms of BMP15, possibly together with other proteins. 
Under non-reducing conditions, there was a consistent very faint band observed at $\sim 35 \mathrm{kDa}$ in the oocyte lysate samples (Figure $3.7 \mathrm{~A} 1$ ). While the molecular weight of the band corresponds with a possible mature BMP15 homodimer, this is unlikely to be the case as the band was not detected in samples that had been crosslinked (Figure $3.7 \mathrm{C} 1$ ). Furthermore, homodimers of BMP15 are believed to be non-covalently bound which means they should be dissociated under non-reducing conditions. There were also no consistent bands in the $\sim 60-150 \mathrm{kDa}$ region, and thus there was no clear evidence of homodimer (BMP15-BMP15) or heterodimer (BMP15- GDF9) formation involving mature or promature BMP15 proteins in rat oocytes. No BMP15 in any molecular form was detected in the oocyte conditioned media samples across all three conditions, even when the blots were overexposed (Figure 3.7).

In the recombinant HEK-293 produced pig BMP15 conditioned media (positive control), both promature and mature BMP15 were detected under reducing conditions, with or without crosslinking ( $\sim 50 \mathrm{kDa}$ and $\sim 12 \mathrm{kDa}$, respectively), and under non-reducing conditions ( $\sim 2 \mathrm{kDa}$ and $\sim 15 \mathrm{kDa}$, respectively). Significantly more mature, than promature BMP15 was detected (Figure 3.7 A1, B1 and C1). For reference, the results of Figure 3.6 (described in Section 3.5) indicated that in the $2 \mu \mathrm{L}$ loaded in the positive control lane of each blot there was approximately $1.16 \mathrm{ng}$ of mature BMP15 present. Following crosslinking of the recombinant pig BMP15, there was evidence of a possible non-covalent mature BMP15 homodimer ( $28-30 \mathrm{kDa}$; Figure $3.7 \mathrm{C} 1$ ). The positive control also contained an unknown band at $\sim 74 \mathrm{kDa}$ which was present in the crosslinked and non-crosslinked recombinant pig BMP15 under reducing conditions (Figure 3.7 B1 and C1). However a similar sized band was also observed in the negative control (recombinant HEK-293produced pig GDF9 conditioned media) under reducing conditions, which indicates that this band may be a non-specific target which is expressed by HEK-293 cells during recombinant protein production. The recombinant pig BMP15 positive control also had two additional unknown bands at $\sim 150-250 \mathrm{kDa}$ that were only present only when crosslinked (Figure 3.7 C1). These bands do not correspond with the molecular weight of any known dimer forms of BMP15, but may represent multimers of BMP15 with other proteins. Under nonreducing conditions, the positive control showed two very faint unknown bands at $\sim 90-120$ kDa which could possibly represent a homodimer of promature BMP15 (Figure 3.7 A1). 
However, the absence of this band in the crosslinked blot (Figure $3.7 \mathrm{C} 1$ ) makes this unlikely. Furthermore, all evidence in the literature to date indicates that the dimer formed between two promature monomers of BMP15 is non-covalent, which means any homo or heterodimers should dissociate when exposed to SDS in the non-reducing loading buffer. There were no bands visible in the negative control lane aside from the one faint band at $\sim 74 \mathrm{kDa}$ under reducing conditions which was mentioned earlier.

The preabsorption of the Mab61A antibody with E.coli-produced ovine BMP15 resulted in no visible bands under any conditions (Figures 3.7). This adds weight to the assumption that the bands observed were due to specific binding of the primary antibody to BMP15. 

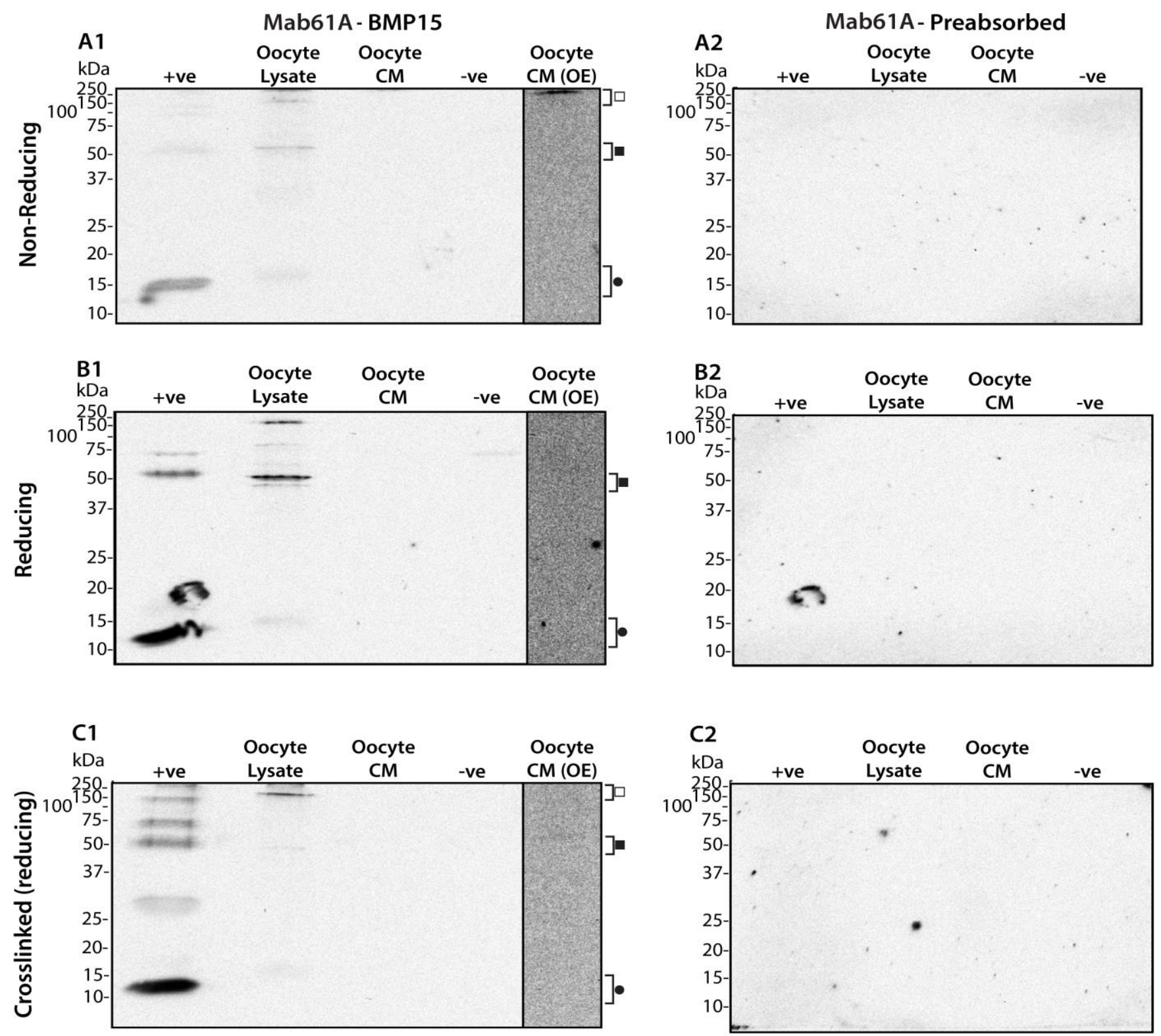

Figure 3.7: The molecular forms of BMP15 present in, and secreted from, rat oocytes. (A1), (B1) and (C1) represent Western blots performed under non-reducing conditions, reducing conditions and reducing conditions where the proteins were crosslinked, respectively. In each blot, Lane 1 contains $2 \mu \mathrm{L}$ of recombinant HEK-293-produced pig BMP15 conditioned media (positive control), Lane 2 contains the lysate from 200 rat oocytes, Lane 3 contains the conditioned media (CM) from 250 rat oocytes, Lane 4 contains $2 \mu \mathrm{L}$ of recombinant HEK-293-produced pig GDF9 conditioned media (negative control). Lane 5, separated by a black border, represents the results from Lane 3 which have been exposed for $4 x$ the original exposure time, and have had black levels increased in an attempt to increase the sensitivity of band detection in the oocyte conditioned media samples. (A2), (B2) and (C2) represent the blots of (A1), (B1), and (C1), respectively after they have been stripped and re-probed with Mab61A that had been preabsorbed with $3 \times \mathrm{w} / \mathrm{w}$ E.coli-produced ovine BMP15. The blots shown are representative of three independent replicates, each of which produced similar results. 


\subsection{The molecular forms of BMP15 in, and secreted from, oocytes of sheep (litter size 1-3)}

A series of Western blots were performed using the sheep oocyte lysate and sheep oocyte conditioned media samples. Representative Western blots are shown in Figure 3.8. A total of 100 lysed sheep oocytes or conditioned media from culturing 125 sheep oocytes were used per lane.

Bands representing promature and mature BMP15 were observed in the oocyte lysates under all conditions (non-reducing, reducing and crosslinked; Figures 3.8 A1, B1 and C1, respectively). The promature bands were considerably denser than the mature BMP15 bands in each case. Promature BMP15 appeared as two high density bands in the oocyte lysate samples under reducing (with or without crosslinking) ( $\sim 44-48$ kDa; Figures 3.8 B1 and $\mathrm{C} 1$ ), and non-reducing ( 47-51 kDa; Figure $3.8 \mathrm{~A} 1$ ) conditions. The same band shift observed between reducing and non-reducing conditions was also observed in rats (Figure 3.7), pigs (Figure 3.9) and deer (Figure 3.10), and is described in more detail in Section 3.6. Mature BMP15 was observed in oocyte lysates as a single band of moderate density under reducing (with or without crosslinking) ( $\sim 13 \mathrm{kDa}$; Figures $3.8 \mathrm{~B} 1$ and $\mathrm{C} 1$ ) and non-reducing conditions ( 16-17 kDa; Figure 3.8 A1). The single 16kDa band directly above the mature band in the oocyte lysate sample on the reducing blot was not observed in the other two replicates (Figure $3.8 \mathrm{~B} 1$ ).

In the oocyte conditioned media, faint single/double bands representing promature BMP15 were observed under reducing and non-reducing conditions ( $\sim 44-51 \mathrm{kDa}$; Figures 3.8 A1 and B1), however a promature band was not visible once the samples were crosslinked (Figure 3.8 C1). It is possible that the promature protein band in the crosslinked (reducing) sample was below detection limits due to the low levels of protein in the oocyte conditioned media samples. Mature BMP15 appeared as faint bands with similar molecular weights to the mature bands observed in the oocyte lysate samples. The forms of protein observed in the positive control were identical under each condition to those described in Section 3.6. 
The oocyte lysate samples had a laddering of unknown bands observed around the promature band which was particularly prominent under reducing conditions (Figure 3.8 B1). The absence of these bands following crosslinking (Figure $3.8 \mathrm{C} 1$ ) suggests that it was unlikely to be due to complex formation involving BMP15 with either itself or other proteins. A smear of unknown bands also appeared between 60-150 kDa under each condition. The position of bands in this region varied between replicates, and so they were not interpreted further. The lack of a consistent band at $100 \mathrm{kDa}$ following crosslinking (Figure $3.8 \mathrm{C} 1$ ) means there was no evidence for the presence of promature BMP15 homodimers or heterodimers in either the oocyte lysate or oocyte conditioned media. In either the oocyte conditioned media or the oocyte lysates, there were no bands visible in the $\sim 25-30 \mathrm{kDa}$ region following crosslinking providing no evidence to support the presence of mature BMP15 homodimers or heterodimers (Figure $3.8 \mathrm{C} 1$ ). There was a band in this region in the oocyte lysate samples under reducing conditions (Figure 3.8 B1), however this band is unlikely to represent a dimer as the non-covalent dimers should have dissociated under the reducing conditions, and is not present following cross-linking (Figure $3.8 \mathrm{C} 1$ ).

In the oocyte lysate samples there were bands between 150-250 kDa which consistently appeared in the non-reducing and cross-linked (reducing) oocyte lysate samples (Figures 3.8 $\mathrm{A} 1$ and $\mathrm{C} 1$, respectively), but were less obvious under reducing conditions (Figure 3.1 B1). Similar sized bands were also present in the oocyte conditioned media samples under non reducing conditions. These bands may indicate the presence of large covalently and non-covalently bound protein complexes that includes multimers of the different forms of BMP15 together with other proteins.

The preabsorption of the Mab61A antibody with E.coli-produced ovine BMP15 resulted in an absence of bands (Figure 3.8 A2, B2 and C2) which supports the assumption that bands observed in the Mab61A labelled blots were due to specific binding of the primary antibody. 
Mab61A - BMP15

Mab61A - Preabsorbed

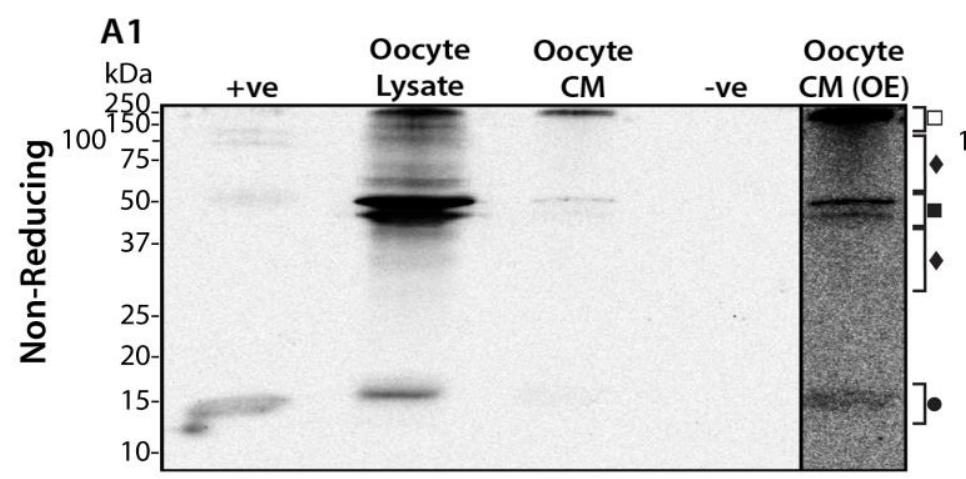

A2 Oocyte Oocyte
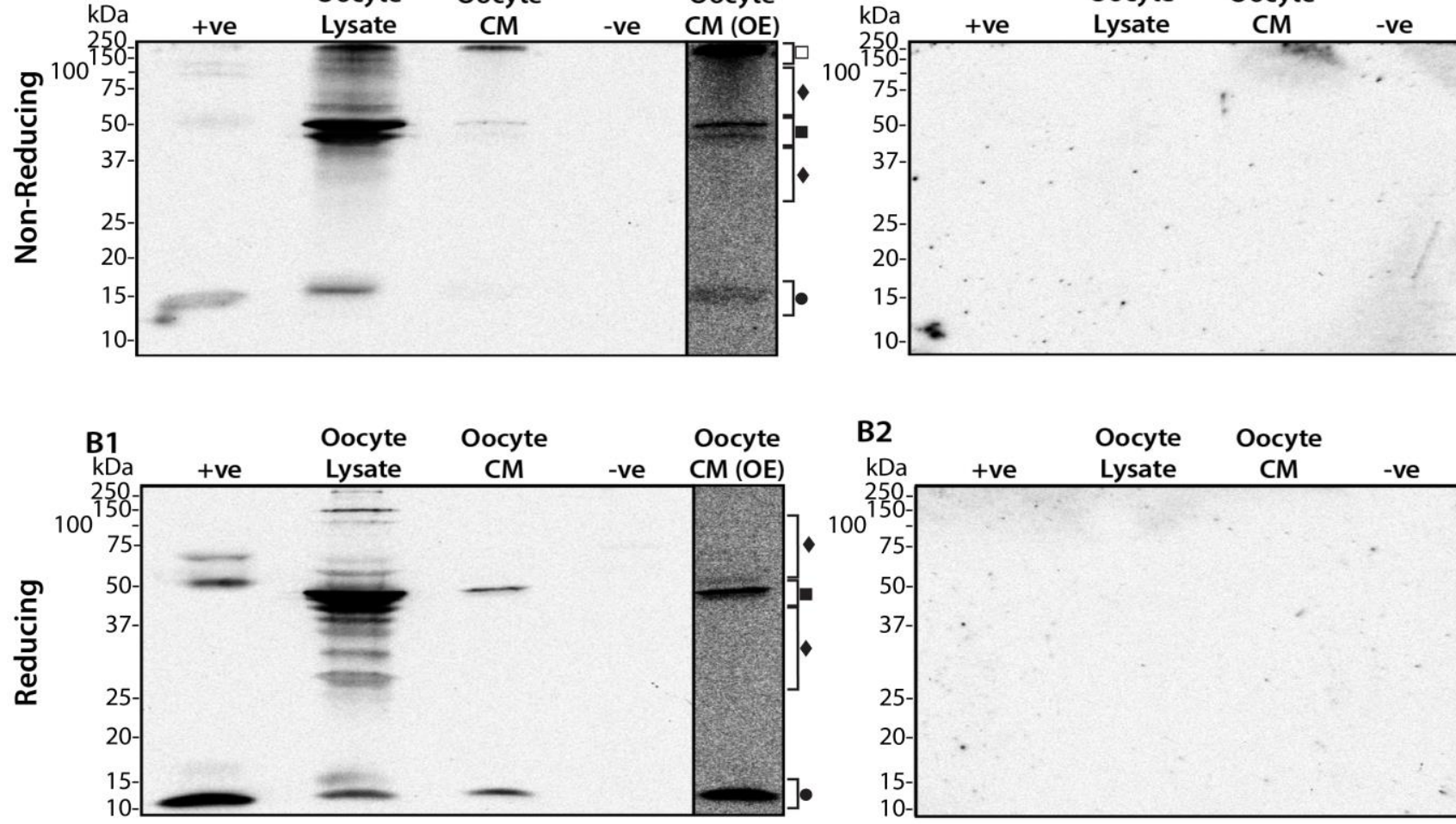

$\mathrm{kDa}$ +ve Lysate CM -ve

100
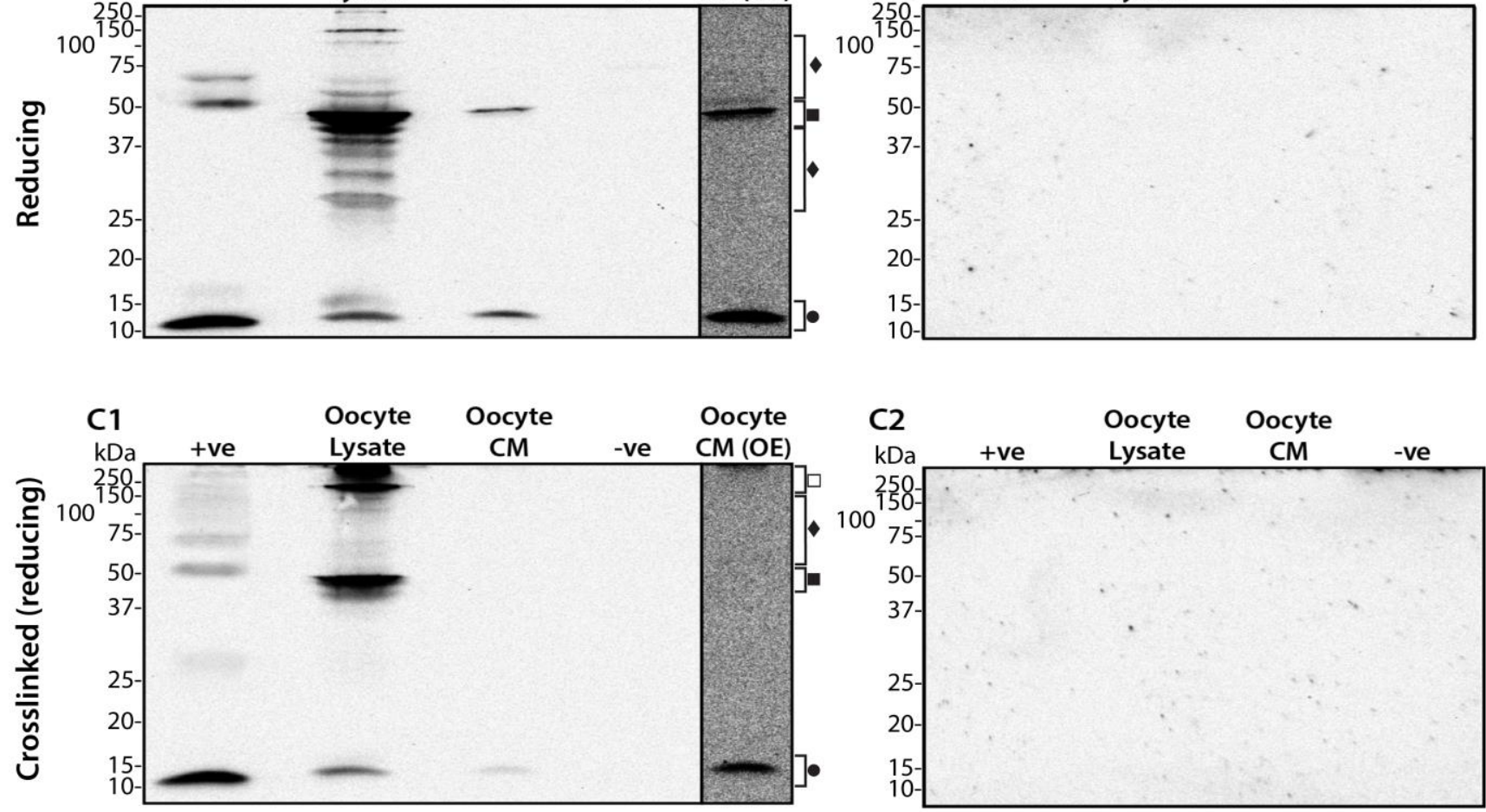

Figure 3.8: The molecular forms of BMP15 present in, and secreted from sheep oocytes. (A1), (B1) and (C1) represent Western blots performed under non-reducing conditions, reducing conditions, and reducing conditions where the proteins were crosslinked, respectively. In each blot, Lane 1 contains $2 \mu \mathrm{L}$ of recombinant HEK-293 produced pig BMP15 conditioned media (positive control), Lane 2 contains the lysate from 100 sheep oocytes, Lane 3 contains the conditioned media from 125 sheep oocytes, Lane 4 contains $2 \mu \mathrm{L}$ of recombinant HEK-293 produced pig GDF9 conditioned media (negative control). Lane 5, separated by a black border, represents the results from Lane 3 that have been exposed for $4 \mathrm{x}$ the original exposure time, and have had black levels increased in an attempt to increase the sensitivity of band detection in the oocyte conditioned media samples. (A2), (B2) and (C2) represent the blots of (A1), (B1), and (C1) respectively, after they have been stripped and re-probed with Mab61A which had been preabsorbed with 3x w/w E.coli-produced ovine BMP15. The blots shown are representative of three independent replicates, each of which produced similar results. 


\subsection{The molecular forms of BMP15 in, and secreted from, oocytes of pigs (litter size 14-22)}

The same series of Western blots performed in Figures 3.7 and 3.8 were also performed using the oocytes of pigs (Figure 3.9). A total of 80 and 100 pig oocytes were used per lane for oocyte lysate and oocyte conditioned media samples, respectively.

Promature and mature BMP15 were observed in the oocyte lysate samples under all conditions (Figures 3.9 A1, B1 and C1, respectively). Promature BMP15 appeared as one or two high density $\sim 49-52 \mathrm{kDa}$ bands in the oocyte lysate samples under all three conditions. Interestingly, although the apparent molecular weight of the promature protein did appear slightly larger under non-reducing conditions compared to reducing conditions, the shift was not as pronounced as what was observed in the other three species. Mature BMP15 appeared as a single band of moderate density under reducing conditions, with or without crosslinking ( $11-12 \mathrm{kDa}$; Figures $3.9 \mathrm{~B} 1$ and $\mathrm{C} 1$ ), and under non-reducing conditions ( $15-$ 16 kDa; Figure 3.9 A1).

In the oocyte conditioned media samples under all three conditions there were faint single bands representing promature and mature BMP15, each of which had the same molecular weights as the equivalent bands in the oocyte lysate samples (Figure 3.9 A1, B1 and C1). The forms of protein observed in the recombinant HEK-293 produced pig BMP15 conditioned media (positive control) were identical under each condition to those described in Section 3.6.

There was a faint laddering of unknown bands around the promature band in the oocyte lysate samples under reducing conditions (Figure 3.9 B1), but absent under the other two conditions (Figure 3.9 A1 and C1). A similar laddering effect was observed in the oocyte lysates of sheep and deer (Figures 3.8 and 3.10); however, in their case the bands were much more prominent. The absence of these bands following crosslinking (Figure 3.9 C1) suggests they are unlikely to be due to any BMP15 complex involving either another BMP15 molecule or other proteins. 
A smear of unknown bands appeared between $\sim 60-150 \mathrm{kDa}$ in the oocyte lysate samples under each condition, similar to what was observed in the oocyte lysates of sheep and deer (Figures 3.8 and 3.10). As was the case with the sheep and deer samples, the position of bands in this region varied between replicates of the pig oocyte blots, and so they were not interpreted further. There was no consistent band at $\sim 100 \mathrm{kDa}$, or at $\sim 25-30 \mathrm{kDa}$ following sample crosslinking (Figure $3.9 \mathrm{C} 1$ ) thus there is no supporting evidence for the presence of either promature or mature BMP15 homodimers or heterodimers either within or secreted from the oocyte. There were bands between $\sim 150-250 \mathrm{kDa}$ in the oocyte lysate and oocyte conditioned media samples under non-reducing conditions and following crosslinking (Figure 3.9 A1 and C1) that were absent in non-crosslinked samples under reducing conditions (Figure $3.9 \mathrm{~B} 1$ ). These bands were similar to those observed in sheep and deer (Figure 3.8 and 3.10, respectively). As with the other species, these may indicate the presence of large covalently and non-covalently bound multimer complexes.

As observed previously, the preabsorption of the Mab61A antibody with E.coli-produced ovine BMP15 resulted in an absence of the bands detected (Figure 3.9 A2, B2 and C2). This supports the assumption that the bands observed were likely due to specific binding of the antibody. 


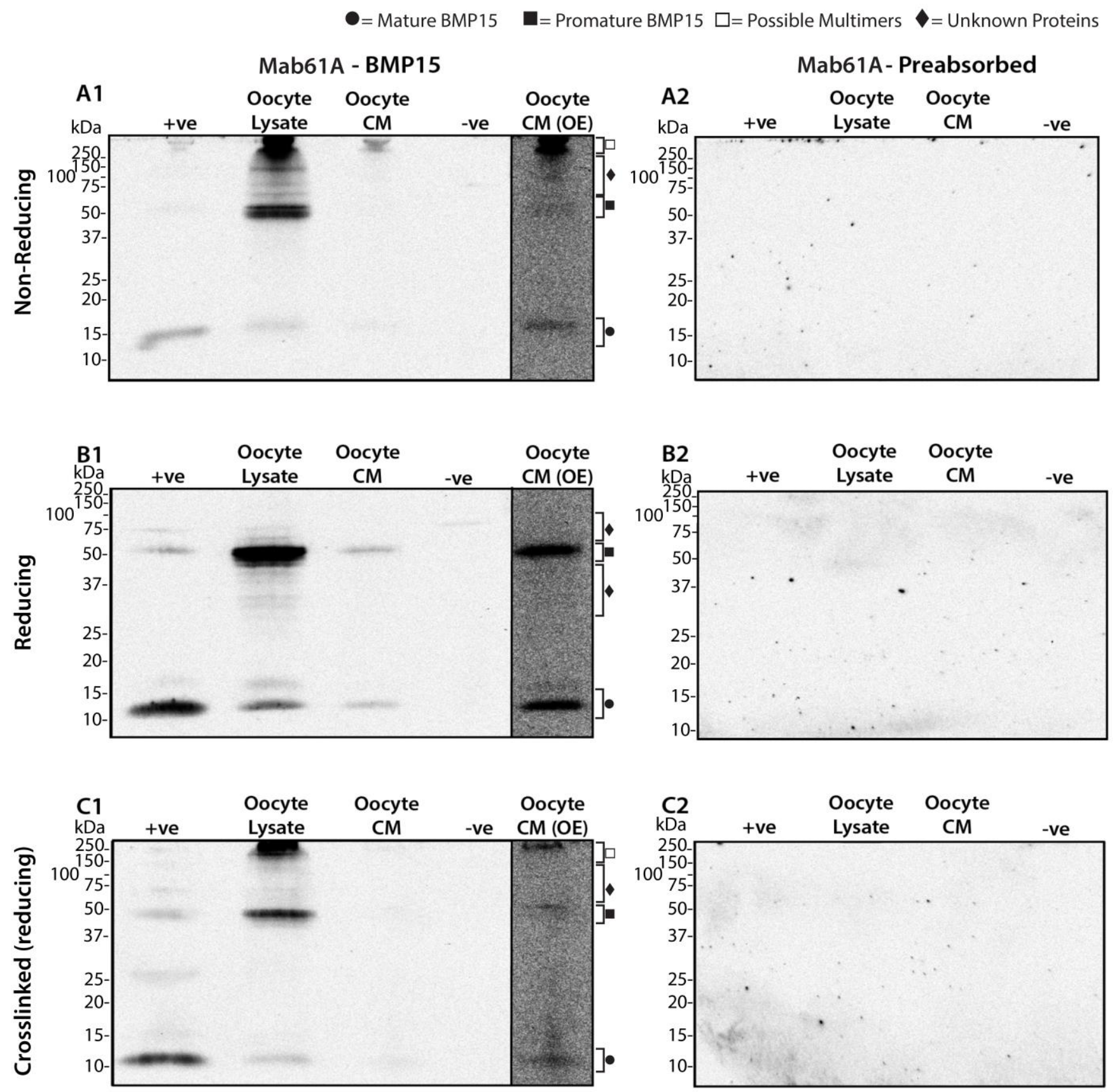

Figure 3.9 The molecular forms of BMP15 present in, and secreted from pig oocytes. (A1), (B1) and (C1) represent Western blots performed under non-reducing conditions, reducing conditions, and reducing conditions where the proteins were crosslinked, respectively. In each blot, Lane 1 contains $2 \mu \mathrm{L}$ of recombinant HEK-293 produced pig BMP15 conditioned media (positive control), Lane 2 contains the lysate from 80 pig oocytes, Lane 3 contains the conditioned media from 100 pig oocytes, Lane 4 contains $2 \mu \mathrm{L}$ of recombinant HEK-293 produced pig GDF9 conditioned media (negative control). Lane 5, separated by a black border represents the results from Lane 3 which have been exposed for $4 x$ the original exposure time, and have had black levels increased in an attempt to increase the sensitivity of band detection in the oocyte conditioned media samples. (A2), (B2) and (C2) represent the blots of (A1), (B1), and (C1), respectively (without the overexposed conditioned media lane) after they have been stripped and re-probed with Mab61A which had been preabsorbed with 3x w/w E.coli-produced ovine BMP15. The blots shown are representative of three independent replicates, each of which produced similar results. 


\subsection{The molecular forms of BMP15 in, and secreted from, oocytes of red deer (litter size $=1$ )}

The same series of Western blots performed in Figures 3.7, 3.8 and 3.9 were performed using deer oocytes (Figure 3.10). A total of 100 and 125 deer oocytes were used for oocyte lysate and oocyte conditioned media samples per lane respectively.

Both promature and mature BMP15 was present in the oocyte lysate and oocyte conditioned media samples under all three conditions tested (Figures $3.10 \mathrm{~A} 1, \mathrm{~B} 1$ and C1). There appeared to be more promature BMP15 present than mature BMP15 in the deer oocyte lysate samples (Figure 3.10), which was similar to what was observed in rat, sheep and pig oocytes (c.f. Figures 3.7, 3.8 and 3.9). The oocyte conditioned media samples, had significantly less BMP15 present than the oocyte lysate samples, but both molecular forms could be detected under all three conditions (Figure $3.10 \mathrm{~A} 1, \mathrm{~B} 1$ and $\mathrm{C} 1$ ). In the oocyte lysate samples, promature BMP15 appeared as two high density bands under reducing conditions, with or without crosslinking (Figure 3.10 B1 and C1; $\sim 43-49$ kDa), and under non-reducing conditions (Figure $3.10 \mathrm{~A} 1$; 45-51 kDa). Mature BMP15 appeared as a single medium density band at $\sim 13-14 \mathrm{kDa}$ under reducing conditions (with or without crosslinking), and $\sim 16 \mathrm{kDa}$ under non-reducing conditions. In the oocyte conditioned media samples, the promature and mature bands were much fainter, and appeared as single or double bands with similar molecular weights to the equivalent bands in the oocyte lysate samples. The apparent molecular weight shift of the promature and mature bands under non-reducing conditions compared to reducing conditions was similar to what was observed in sheep and rats (Figures 3.7 and 3.8, described in Section 3.6). The forms of protein observed in the recombinant HEK-293 produced pig BMP15 conditioned media positive control were identical under each condition to those described in Section 3.6.

There was a laddering of high density unknown bands around the promature band in the oocyte lysate samples under reducing conditions, and to a lesser extent under nonreducing conditions (Figure $3.10 \mathrm{~B} 1$ and $\mathrm{A} 1$, respectively) which was similar to what was observed in the oocyte lysates of sheep (Figure 3.8). The high density and close proximity of these unknown bands under reducing conditions resulted in the coagulation of signal 
shown in Figure 3.10. However, when densitometric analysis was performed for later figures (Figures 3.13-3.15), a lower exposure of the blots were used for band separation. The laddering effect is lessened after sample crosslinking (Figure $3.10 \mathrm{C} 1$ ). This makes it unlikely that these bands are due to any sort of BMP15 complex. The distinct band at $\sim 30 \mathrm{kDa}$ in the oocyte lysate under non-reducing conditions (Figure $3.10 \mathrm{~A} 1$ ) was not present in either of the other two replicates so was not interpreted any further. There was a smear of unknown bands between $~ 60-150 \mathrm{kDa}$ in the oocyte lysate samples under each condition (Figure 3.10), similar to what was observed in the oocyte lysates of sheep and pigs (Figures 3.8 and 3.9). Once again, there were no distinct bands observed consistently across replicates in this region so no firm conclusions could be drawn about the proteins present. The absence of a consistent band at $\sim 100 \mathrm{kDa}$, or at $\sim 25-30 \mathrm{kDa}$ following sample crosslinking (Figure $3.10 \mathrm{C} 1$ ) means there is no supporting evidence for the presence of either promature or mature BMP15 homodimers or heterodimers either within or secreted from the oocyte. There were bands between $\sim 150-250 \mathrm{kDa}$ in the oocyte lysate samples under non-reducing conditions and reducing conditions with crosslinking (Figure $3.10 \mathrm{~A} 1$ and $\mathrm{C} 1$ ). These bands were less marked under reducing conditions without crosslinking (Figure $3.10 \mathrm{~B} 1$ ). This observation was consistent amongst replicate blots, and was similar to what was observed in the oocyte lysates of the sheep and pig (Figures 3.8 and 3.9, respectively). Similar bands were observed in the oocyte conditioned media samples (Figure 3.10). As with the other species, these bands may indicate the possible presence of large covalently and non-covalently bound BMP15 complexes.

Probing blots with Mab61A that had been preabsorbed with E.coli-produced ovine BMP15 resulted in no detectable bands (Figure 3.10 A2, B2, C2), which was similar to what was observed in rat, sheep and pig oocytes (Figures 3.7, 3.8, and 3.9, respectively). A faint band at $\sim 45 \mathrm{kDa}$ was most likely due to the incomplete stripping of the dense promature BMP15 band. The fact that the bands all disappeared (or faded dramatically) following preabsorption of the primary antibody suggests that the bands observed in Figures 3.10 A1, B1 and C1 were most likely due to specific binding of the primary antibody to deer BMP15. 

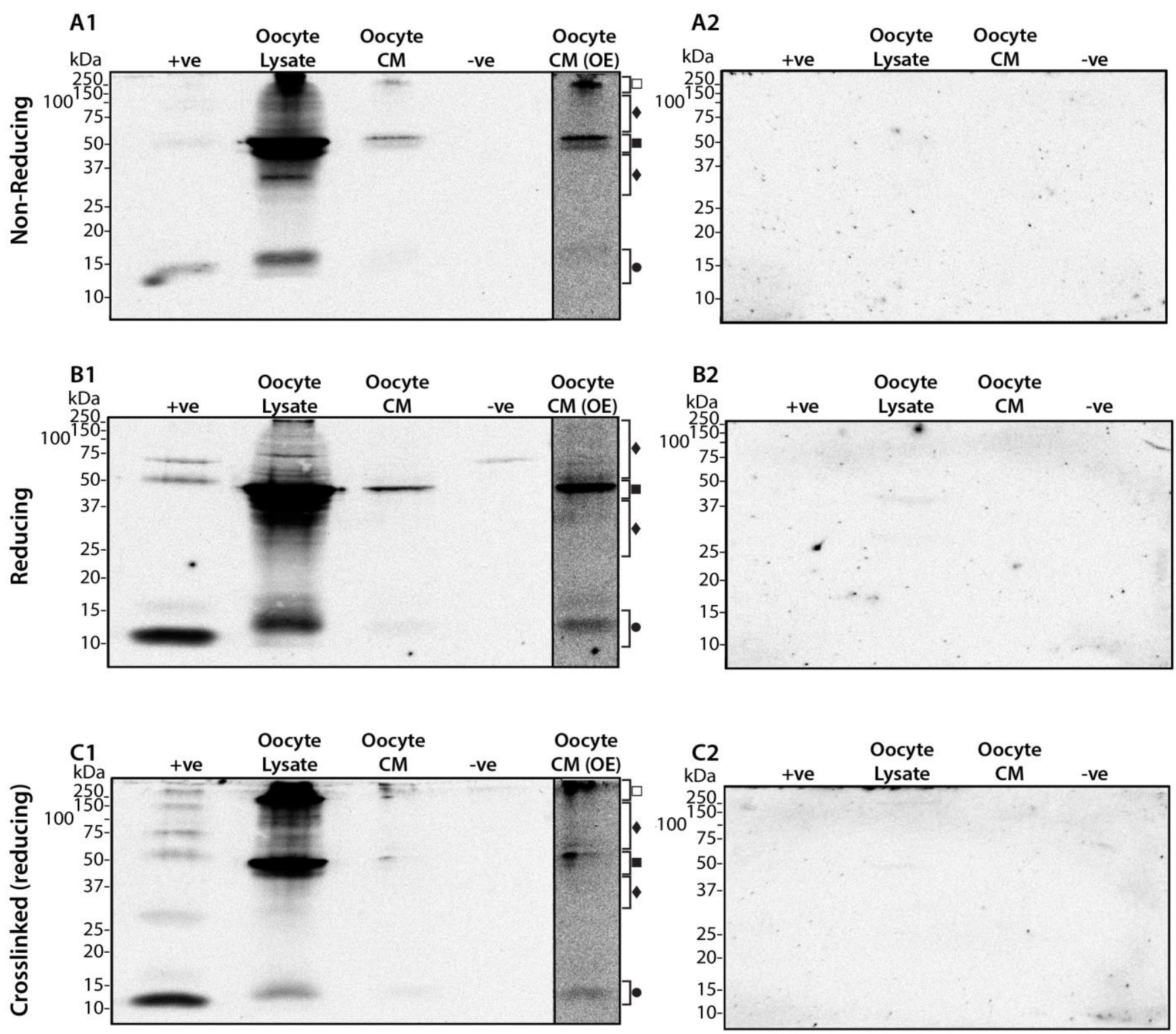

Figure 3.10: The molecular forms of BMP15 present in, and secreted from deer oocytes. (A1), (B1) and (C1) represent Western blots performed under non-reducing conditions, reducing conditions, and reducing conditions where the proteins were crosslinked, respectively. In each blot, Lane 1 contains $2 \mu \mathrm{L}$ of recombinant HEK-293 produced pig BMP15 conditioned media (positive control), Lane 2 contains the lysate from 100 deer oocytes, Lane 3 contains the conditioned media from 125 deer oocytes, Lane 4 contains $2 \mu \mathrm{L}$ of recombinant HEK-293 produced pig GDF9 conditioned media (negative control). Lane 5, separated by a black border, represents the results from lane 3 which have been exposed for $4 x$ the original exposure time, and have had black levels increased in an attempt to increase the sensitivity of band detection in the oocyte conditioned media samples. (A2), (B2) and (C2) represent the blots of (A1), (B1), and (C1), respectively (without the overexposed conditioned media lane) after they have been stripped and re-probed with Mab61A which had been preabsorbed with 3x w/w E.coli-produced ovine BMP15. The blots shown are representative of three independent replicates, each of which produced similar results. 


\subsection{The alignment of intra-oocyte and oocyte-secreted molecular forms of BMP15 with those of GDF9 in rats, sheep, pigs and deer}

In order to determine whether any of the bands detected in the oocyte lysate or oocyte conditioned media samples were due to interactions of BMP15 with GDF9 at a molecular level, one of the replicate blots demonstrated in Figures 3.7-3.10 was stripped and relabelled with Mab37A (Figure 3.11). Mab37A is a monoclonal antibody targeting the mature GDF9 peptide. The blots that were stripped and labelled with Mab37A had already previously been stripped and re-labelled the anti- $\beta$-actin antibody. The band observed at $\sim 42-46 \mathrm{kDa}$ in all the GDF9 oocyte lysate blots, which was particularly prominent in the deer oocyte lysate blots, was likely due to incomplete stripping of the dense $\beta$-actin band. Evidence for this was provided by overlaying the $\beta$-actin labelled blots with the GDF9 labelled blots whereby the bands aligned perfectly (data not shown). The reader should not be misled into comparing the relative apparent densities of the BMP15 bands with the GDF9 bands. The blots that were immunoprobed for GDF9 underwent a longer exposure time during imaging than the BMP15 blots to compensate for the loss of protein during stripping. Due to this manipulation, the blots may only be used to compare the relative molecular weights of the bands rather than the band intensities. The purpose of these blots was to elucidate whether BMP15:GDF9 interactions exist.

In each blot, promature GDF9 was slightly larger $(\sim 55-60 \mathrm{kDa})$ than that observed for promature BMP15. Mature GDF9 also appeared slightly larger than its BMP15 counterpart, under both reducing ( $\sim 17-20 \mathrm{kDa})$ and non-reducing ( $20-25 \mathrm{kDa})$ conditions. Many of the anti-GDF9 blots of the sheep, pig and deer oocyte lysates had an additional unknown band with the same molecular weight as the mature BMP15 band which may represent slight cross reactivity of Mab37A towards the mature BMP15 protein. The laddering of unknown bands between $\sim 30-45 \mathrm{kDa}$ that was observed the deer pig and sheep oocyte lysate samples (as described in Sections 3.7, 3.8 and 3.9) did not appear to correlate with any similar GDF9 bands. Thus, it is unlikely that these bands were due to the formation of complexes between the two proteins. A faint unknown band observed at $\sim 55-60 \mathrm{kDa}$ in the 
oocyte lysates of many of the BMP15 labelled blots appeared to correlate with the dense promature GDF9 band observed in blots labelled for GDF9. This suggests that the band may be due to potential cross reactivity of Mab61A towards the GDF9 promature protein. However, preabsorption experiments indicated only extremely low cross reactivity towards GDF9 (Figure 3.3), and there was no cross-reactivity observed in the recombinant HEK-293 produced pig GDF9 control in each blot (see appendix IV for representative full blot with control lanes). Furthermore, the $\sim 55-60 \mathrm{kDa}$ band in the BMP15 labelled blot was not consistent across all of the replicates, and therefore it is unlikely that cross-reactivity was the cause of the band. It may simply be a coincidence that the bands share similar molecular weights.

There were no bands observed consistently between the three repeats in the $~ 60-140 \mathrm{kDa}$ region of the BMP15 or GDF9 labelled blots, and so no comparisons could be made between the BMP15 and GDF9 labelled blots in this region. The lack of consistent bands in this region does not completely rule out the possibility of homodimeric or heterodimeric forms of BMP15 and GDF9 in the oocyte lysate samples as there was a smearing of bands in this region for both proteins in the sheep, deer and pig samples. However under the conditions used, there was no evidence to support the existence of these forms both within and secreted by the oocyte. There were, however, consistent anti-BMP15 labelled bands observed in the $\sim 140-212 \mathrm{kDa}$ region in sheep, pig and deer samples under non-reducing conditions and in cross-linked samples under reducing conditions (described in Section 3.7, 3.8 and 3.9). These bands aligned well with similar sized bands in the anti-GDF9 labelled samples, which showed a similar pattern across the three conditions used. This allows for the possibility that the bands represent high molecular weight non-covalently and covalently bound multimeric complexes which include different forms of both BMP15 and GDF9. 

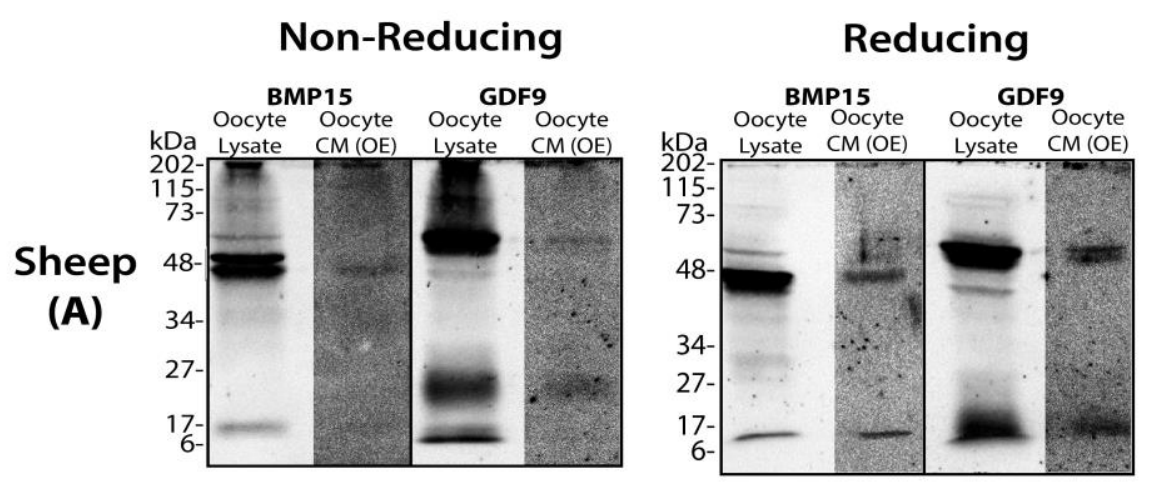

\section{Crosslinked (reducing)}
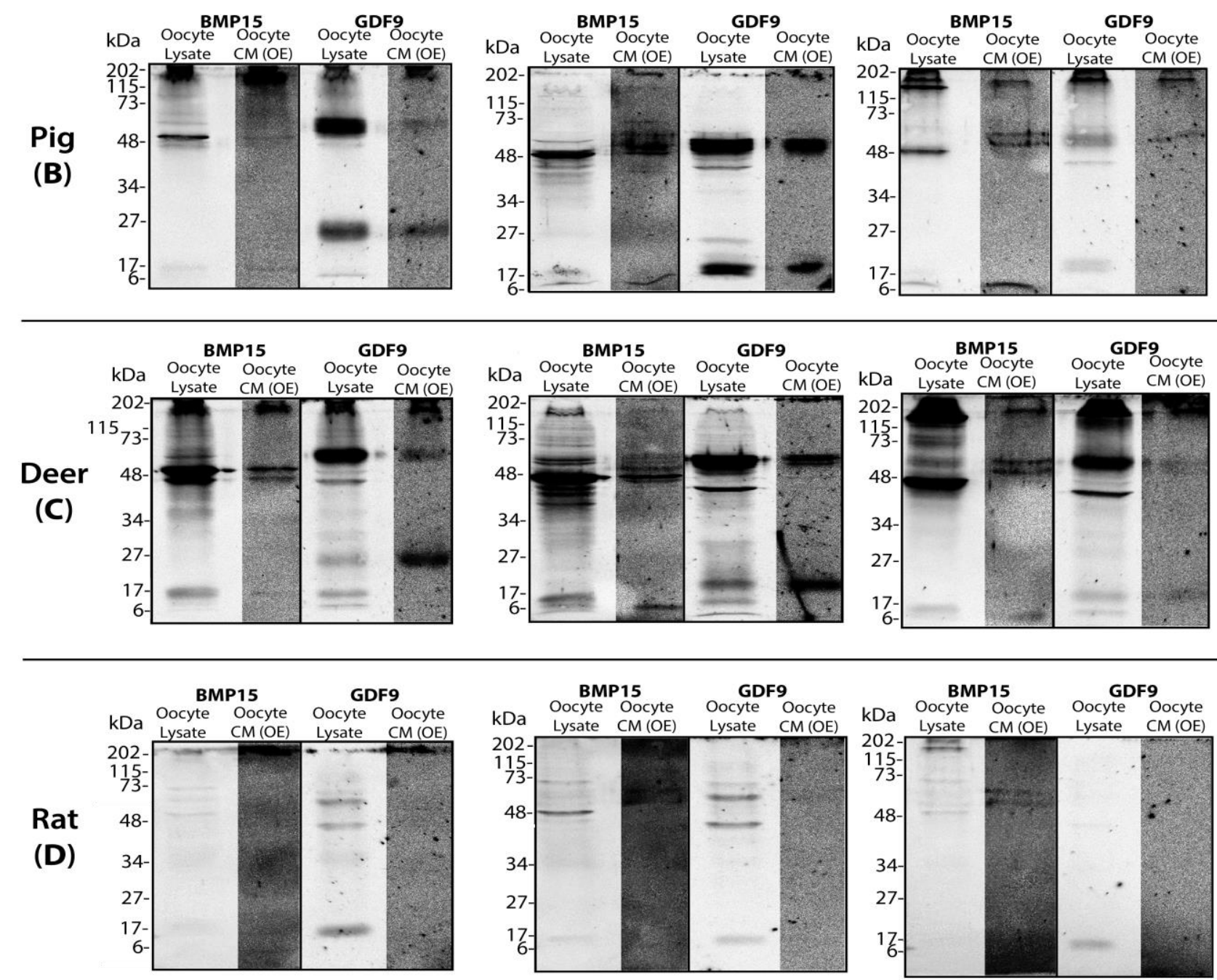

Figure 3.11: Comparisons between the intra-oocyte and oocyte secreted molecular forms of BMP15 and GDF9 detected using Mab61A and Mab37A, respectively. The letters for each blot represent the species used for each blot ( $A=$ sheep, $B=$ pig, $C=$ Deer and $D=$ Rat). The blots were performed under nonreducing conditions, reducing conditions or reducing conditions where the proteins were crosslinked, respectively (indicated by the column header). In each blot, Lanes 1 and 2 have been labelled with the anti-BMP15 antibody Mab61A. Lane 1 represents the oocyte lysate samples for each species. 100 sheep oocytes, 80 pig oocytes, 100 deer oocytes and 200 rat oocytes were used for the oocyte lysate samples. Lane 2 represents the oocyte conditioned media samples for each species and has been overexposed for band detection purposes. 125 sheep oocytes, 100 pig oocytes, 125 deer oocytes and 250 rat oocytes were used for the oocyte conditioned media samples. Lanes 3 and 4 represent Lanes 1 and 2 after having been stripped and re-probed with the anti-GDF-9 antibody Mab37A. A full blot example with all control lanes is provided in Appendix IV. 


\subsection{Models for the quantification and normalisation of the BMP15 band densities across blots}

A series of experiments were performed to determine whether quantification and comparison of both promature and mature BMP15 across blots was possible. The antibodies were used at saturation levels (based on antibody titrations, data not shown). A Western blot containing titrations of pig oocyte lysate was labelled with Mab61A, then stripped and labelled with $\beta$-actin (figure $3.12 \mathrm{~A}$ and $\mathrm{B}$ ). While promature BMP15 bands were detectable in as few as 9 oocyte lysates, mature BMP15 and $\beta$-actin were not detected when fewer than 38 and 19 oocyte lysates were used respectively. The densities of these bands were measured, and plotted to determine the linear dynamic range (Figure $3.12 \mathrm{C}$ ). All of the detectable mature and $\beta$-actin bands showed a strong linear relationship with increasing oocyte number, and the promature BMP15 bands also showed a strong linear relationship between 9 and 150 oocyte lysates $\left(R^{2}>0.99\right.$ ) (Figure $\left.3.12 C\right)$. However, when 300 oocyte lysates were used, the band density was lower than expected and did not conform to the linear trend (data point not plotted). This may indicate that the saturation point for the dense promature BMP15 bands lies between 150 and 300 oocyte lysates in pigs.

The data points in the linear dynamic range from Figure $3.12 \mathrm{C}$ were converted into proportions of the maximum band density and sample amount values and then plotted (figure 3.12 D). There were no significant differences between the linear regressions of each protein and all three proteins aligned well with the predicted quantitative response for a protein below saturation levels. This indicates that the band densities of each protein provide an accurate representation of the relative amounts of protein present, provided the bands fall within the linear dynamic range. For example, if a given sample contains half as much mature BMP15 as another sample, it can be expected to have half the band density. It was not a viable use of time and resources to collect all the oocytes required to perform Figures 3.12 A-D with the oocyte lysates of rats, sheep and deer, or the oocyte conditioned media of each species. Instead it was simply assumed that the values of all bands detected fell within the linear dynamic range provided they were less dense than the promature band found using 300 pig oocyte lysates. 
The results illustrated in Figure 3.12 B-D suggested that $\beta$-actin was a viable option for normalisation of protein levels across blots as it appeared to handle large sample sizes without reaching saturation. When an anti- $\beta$-actin antibody was used against the blots in Figures 3.7- 3.10, $\beta$-actin was detected in the oocyte lysate samples in each blot, but was predictably absent from all the conditioned media samples (a sample full blot is provided in Figure $3.12 \mathrm{~F}$, and the $\beta$-actin band from each blot of one of the repeats is shown in Figure $3.12 \mathrm{E})$. The $\beta$-actin levels were very low in the pig compared to the other three species (Figure $3.12 \mathrm{E}$ ), which is likely due to pig oocytes mostly being recovered in a denuded state (i.e. absent of cumulus cells), compared to that in the other three species.

\subsection{Comparison between the levels of intra-oocyte and oocyte secreted promature and mature forms of BMP15 in rats, sheep, pigs and deer normalised using $\beta$-actin}

Densitometric analysis was performed on the three replicates of the reducing blots (B1) displayed in Figures 3.7 to 3.10 using Image J software. The densities of the promature and mature band found in both the oocyte lysate and oocyte condition media samples were calculated for each species. The blots were then stripped and relabelled for the constitutively active $\beta$-actin protein, which was present in the oocyte lysates of all species (see Figure 3.12 and Section 3.11). The BMP15 band densities were then normalised using the $\beta$-actin band densities according to Section 2.1.6, and the resulting values are presented in Figure 3.13. Only the reducing blots were used for densitometric analysis as they were deemed to give the fairest representation of the absolute levels of promature and mature BMP15 present as they include proteins which may be lost into unknown protein complexes under non-reducing or crosslinked conditions.

In the oocyte lysate samples, there was more promature BMP15 than mature BMP15 in all four species $(P \leq 0.05)$ (Figure $3.13 \mathrm{~A})$. In the oocyte conditioned media samples the difference in levels was much less pronounced. Only the deer exhibited higher levels of 
A

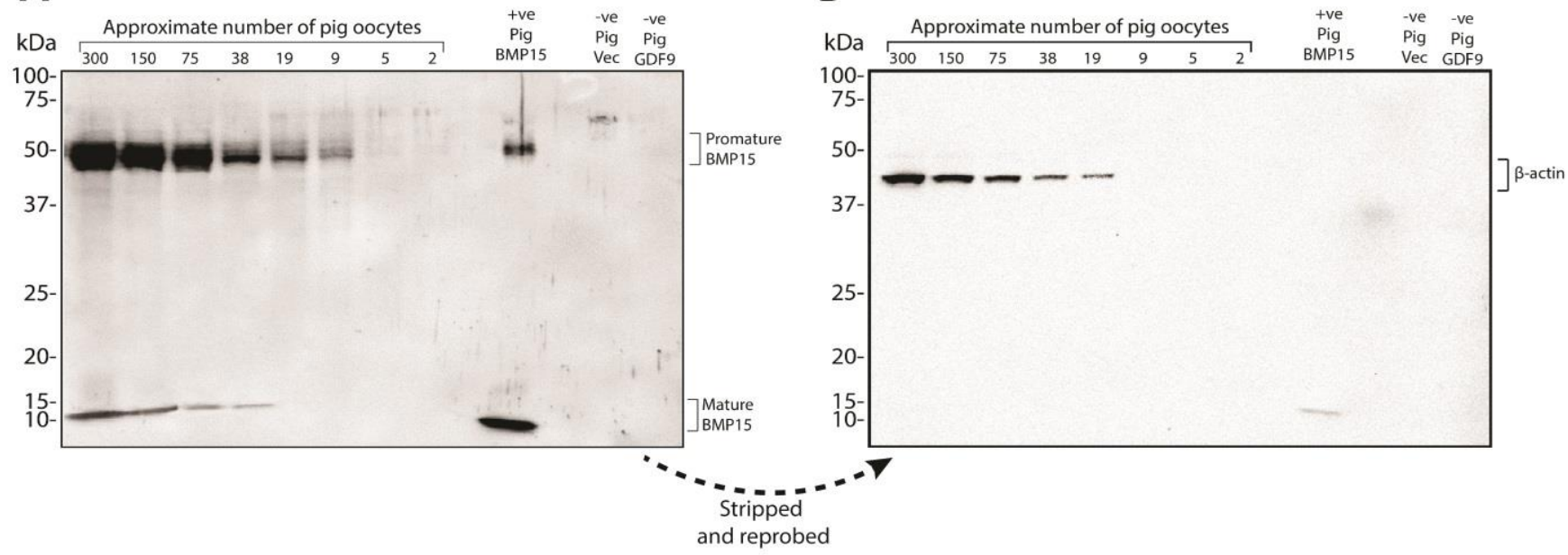

C

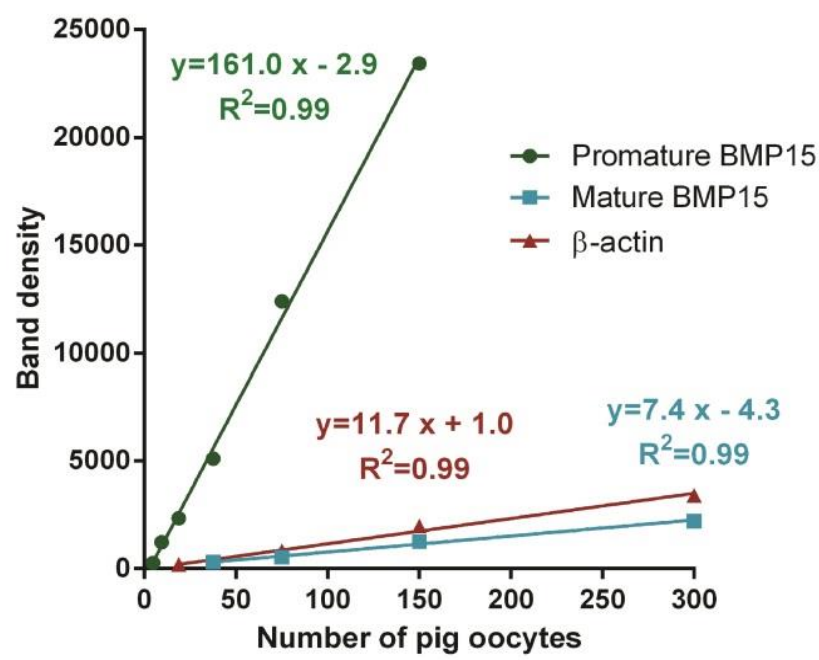

E
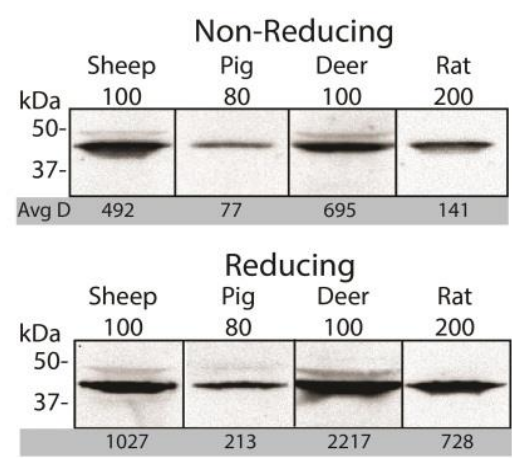

Crosslinked (reducing)

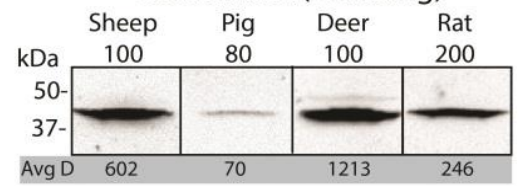

D

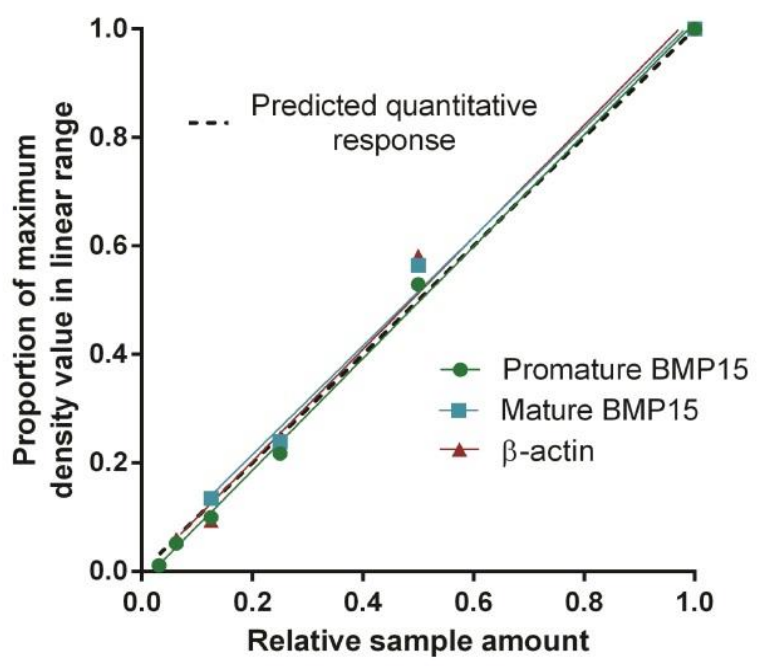

F

Sheep Reducing

(full blot example)

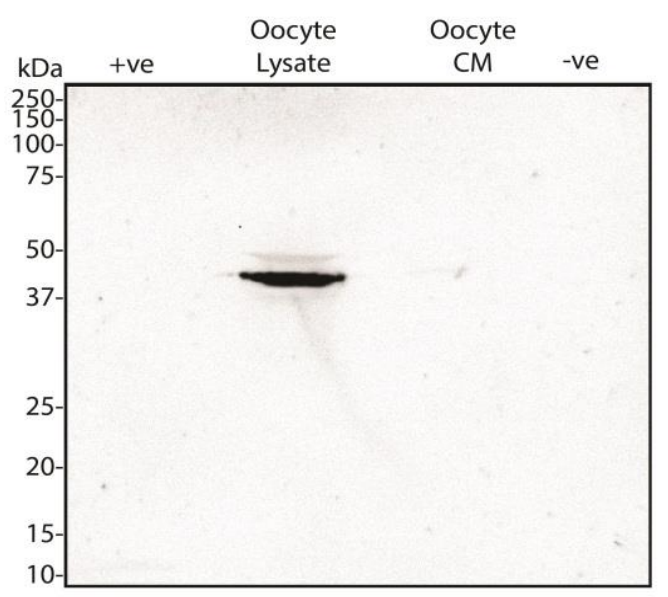


Figure 3.12: Tests performed to investigate reliability of the quantification and normalisation of the BMP15 band densities across blots. (A) Detection of BMP15 in a titration of pig oocyte lysates under reducing conditions using MAB61A. The number of oocytes is indicated above each lane in Lanes 1-8. Lane 9 is a positive control and contains $2 \mu \mathrm{L}$ of recombinant HEK-293 produced pig BMP15. Lanes 10 and 11 are negative controls containing $2 \mu \mathrm{L}$ of untransfected HEK-293 conditioned media and $2 \mu \mathrm{L}$ of recombinant HEK-293 produced pig GDF9 conditioned media, respectively. The position of the promature and mature BMP15 bands are indicated next to the blots (B) The blot from (A) was stripped and reprobed with an anti- $\beta$-actin antibody (Product \# A5441, Sigma Aldrich). The position of the $\beta$-actin bands is indicated next to the blot. (C) The band densities from (A) and (B) were plotted against the number of pig oocytes in the sample. When bands were absent or when the band density fell outside of the linear range the data point was omitted. The data points on the graph were fitted with linear regression lines and the equations and $R^{2}$ values shown are allocated according to the colour and key provided in the figure. (D) The band densities from (C) were converted into a proportion of the highest band density for each given protein type, and the number of oocytes was converted into a proportion of the highest number of oocytes in the linear range for each protein shown in (C). The values were plotted and fitted with linear regression lines. The black dotted line represents the predicted quantitative response whereby an increase in sample causes a proportional increase in the band density. Each linear regression shown had an $\mathrm{R}^{2}$ greater than 0.98 . There was no significant difference between the linear regressions of each of the proteins and the predicted quantitative response. (E) The $\beta$-actin bands detected in the oocyte lysates of each species under non-reducing conditions, reducing conditions, and finally conditions where the proteins were crosslinked before being run under reducing conditions. The species type and number of oocyte lysates used is indicated above each band. The numbers in the grey shaded area underneath each band represent the average density units for the band across three replicate experiments. (F) An example of a full blot (sheep oocyte lysate, reducing conditions) from (D). Lane 1 contains $2 \mu \mathrm{L}$ of recombinant HEK-293 produced pig BMP15 conditioned media (positive control), Lane 2 contains the lysate from 100 sheep oocytes, Lane 3 contains the conditioned media from 125 sheep oocytes, Lane 4 contains $2 \mu \mathrm{L}$ of recombinant HEK-293 produced pig GDF9 conditioned media (negative control).

promature than mature BMP15 $(\mathrm{P}<0.05)$, and no differences were observed in sheep and pigs (Figure $3.13 \mathrm{~B}$ ). There were no bands for either protein observed in the rat oocyte conditioned media samples across any of the replicates.

The levels of each molecular form were also compared across the four species. Overall, there were species effects on the levels of promature or mature BMP15 in the oocyte lysates samples ( $P \leq 0.0001)$, and the oocyte conditioned media samples $(P \leq 0.01)$ (see Figure $3.13 \mathrm{~A}$ and $\mathrm{B})$. In the oocyte lysate samples, pigs had significantly more promature and mature BMP15 than the other three species $(P<0.05)$ (BMP15: $\beta$-actin ratio of 11.8 and 0.72 , respectively), followed by deer (BMP15: $\beta$-actin ratios of 6.9 and 0.26 , respectively) and sheep (BMP15: $\beta$-actin ratio of 4.7 and 0.18 , respectively) which had similar amounts 
to one another. Rat oocyte lysates contained significantly less promature and mature BMP15 than the other three species (BMP15: $\beta$-actin ratio of 0.2 and 0.03 , respectively).

In the oocyte conditioned media samples, pig oocytes secreted the most promature BMP15 $(\mathrm{P}<0.05)$ (BMP15: $\beta$-actin ratio of 0.23$)$, followed by deer (BMP15: $\beta$-actin ratio of 0.06 ) and sheep (BMP15: $\beta$-actin ratio of 0.05 ) which once again had similar amounts to one another. In regards to the mature protein, pigs secreted more than deer $(P<0.05)$ (BMP15: $\beta$-actin ratios of 0.11 and 0.01 respectively), but there was no difference between sheep (BMP15: $\beta$-actin ratio of 0.03 ) and deer, or sheep and pigs. The rat conditioned media was excluded from comparisons because no BMP15 bands were detected.

\subsection{Comparisons between the levels of intra-oocyte and oocyte-secreted promature and mature forms of BMP15 in rats, sheep, pigs and deer with the levels expressed relative to the number of oocytes per sample, and normalised using the mature BMP15 band in the positive control}

There were concerns that standardising the levels of BMP15 across species using $\beta$-actin as described in the previous section may have some limitations. This is because, despite best efforts to denude the oocytes during collection, the oocytes of some species were more successfully denuded than others (as outlined in Section 2.1.2). While all pig oocytes collected were fully denuded, the oocytes of the other three species were collected as a mixture of fully and partially denuded oocytes. The partially denuded oocytes still had a small number of cumulus cells attached and attempts were not made remove these due to the risk of oocyte rupture. The presence of these cumulus cells would have contributed to the $\beta$-actin levels detected in the oocyte lysate samples of the rat, sheep and deer, but not the pig, which could have potentially skewed the results. Thus, an alternative means of comparing the BMP15 levels between species is presented in Figure 3.14 whereby the bands were adjusted for the number of oocytes used per sample, and the levels were 

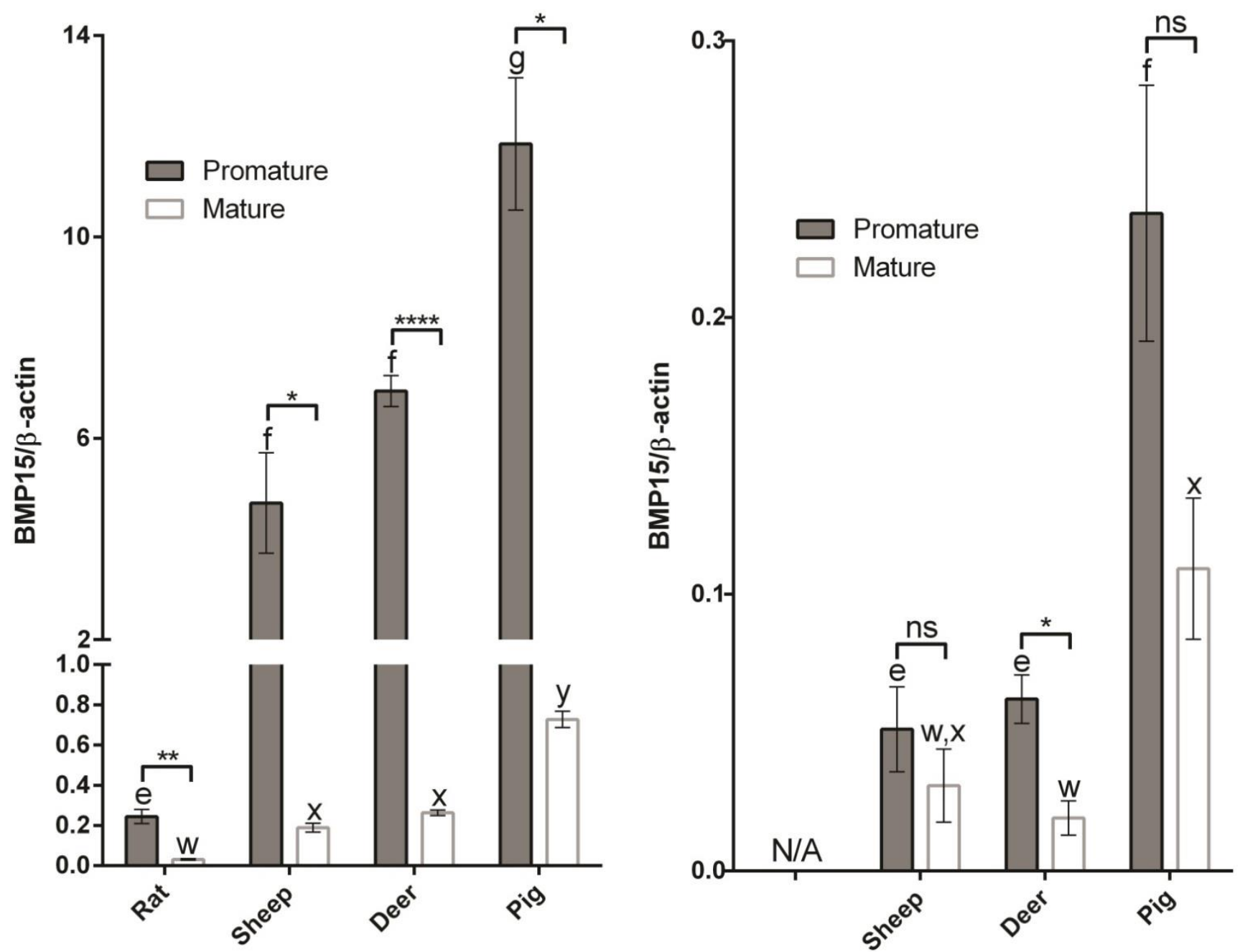

Figure 3.13: Levels of intra-oocyte and oocyte secreted promature and mature BMP15 detected under reducing conditions in each species relative to the $\beta$-actin levels detected for each species. The experiments in Figures 3.6-3.9 were performed in triplicate and the densities of the bands in the reducing blots were measured. The values presented represent the mean target-BMP15 to $\beta$-actin band density ratio. The vertical error bars represent \pm SEM. Statistical analysis was performed according to Section 2.1.6. Different letters above the columns for a given protein form indicate a significant difference between species ( $e, f$ and $g$ are used for the promature protein values and $w, x$ and $y$ are used for the mature protein values) $(P \leq 0.05)$. The same letter above two or more columns indicates no significant difference between them. Brackets indicate significant differences between the promature and mature protein values within each species $\left(*=\mathrm{P} \leq 0.05,{ }^{* *}=\mathrm{P} \leq 0.01, * * * * \mathrm{P} \leq 0.0001\right.$, ns= not significant). N/A= No BMP15 detected. (A) The levels of promature and mature BMP15 in the oocyte lysate of each species. A broken $y$-axis is used due to the low levels of mature protein observed relative to the promature protein. (B) The levels of promature and mature BMP15 detected in the oocyte conditioned media of each species. As there was no $\beta$-actin present in the oocyte conditioned media, the values presented represent the mean ratio between the BMP15 band density and $1.25 \mathrm{x}$ the mean $\beta$-actin band density found in the corresponding oocyte lysate samples on each blot. A multiple of 1.25 was used for the $\beta$-actin band density to adjust for the extra $25 \%$ oocytes used in the conditioned media samples compared to the oocyte lysate sample. 
normalised using the mature BMP15 band in the positive control for each blot (see Section 2.1.6 for details). Once again, only the promature and mature bands from the three replicates of the reducing blots for each species were analysed.

Firstly, the promature and mature BMP15 levels within each species were compared (Figure $3.14 \mathrm{~A}$ and $\mathrm{B}$ ). As expected, the same patterns of intra-species promature versus mature protein levels were observed as previously described (see Section 3.12). When levels were compared between species, there were significant differences in the levels of promature BMP15 in the oocyte lysate samples. When the samples were corrected against oocyte number and the positive control, deer exhibited the most promature BMP15 (adjusted BMP15:+ve control ratio of 20.5), while sheep and pigs had similar levels (adjusted BMP15:+ve control ratio of 6.2 and 5.1, respectively) (Figure 3.14A). The level of promature BMP15 in the rat oocytes was significantly less than that in the other three species (adjusted BMP15:+ve control ratio of $\sim 1$ ). The promature BMP15 levels in the conditioned media samples showed a similar pattern where the deer oocytes contained significantly more promature BMP15 than sheep (adjusted BMP15:+ve control ratios of 0.19 and 0.07 , respectively) $(P \leq 0.05)$ and sheep and pigs had similar levels. However, there was no significant difference between deer and pigs (adjusted BMP15:+ve control ratios of 0.19 and 0.10 , respectively) (Figure $3.14 \mathrm{~B}$ ). There were no bands of promature BMP15 detected across the three replicates in the rat oocyte conditioned media samples.

There were significant differences in the levels of mature BMP15 in the oocyte lysate samples between species (Figure 3.14A). There was more $(P<0.05)$ mature BMP15 in deer oocytes (adjusted BMP15:+ve control ratios of 0.78) compared to sheep and pigs (adjusted BMP15:+ve control ratios of 0.26 and 0.31 , respectively), which were similar. Mature BMP15 levels were lowest $(P<0.05)$ in the oocyte lysate of rats (adjusted BMP15:+ve control ratios of 0.01 ). In the conditioned media samples (Figure $3.14 \mathrm{~B}$ ), mature BMP15 levels were similar between deer, sheep and pigs (adjusted BMP15:+ve control ratios of 0.06, 0.05 and 0.05 , respectively). The rat had the least mature BMP15 in the conditioned media as there were no bands detected in any of the three replicates. 

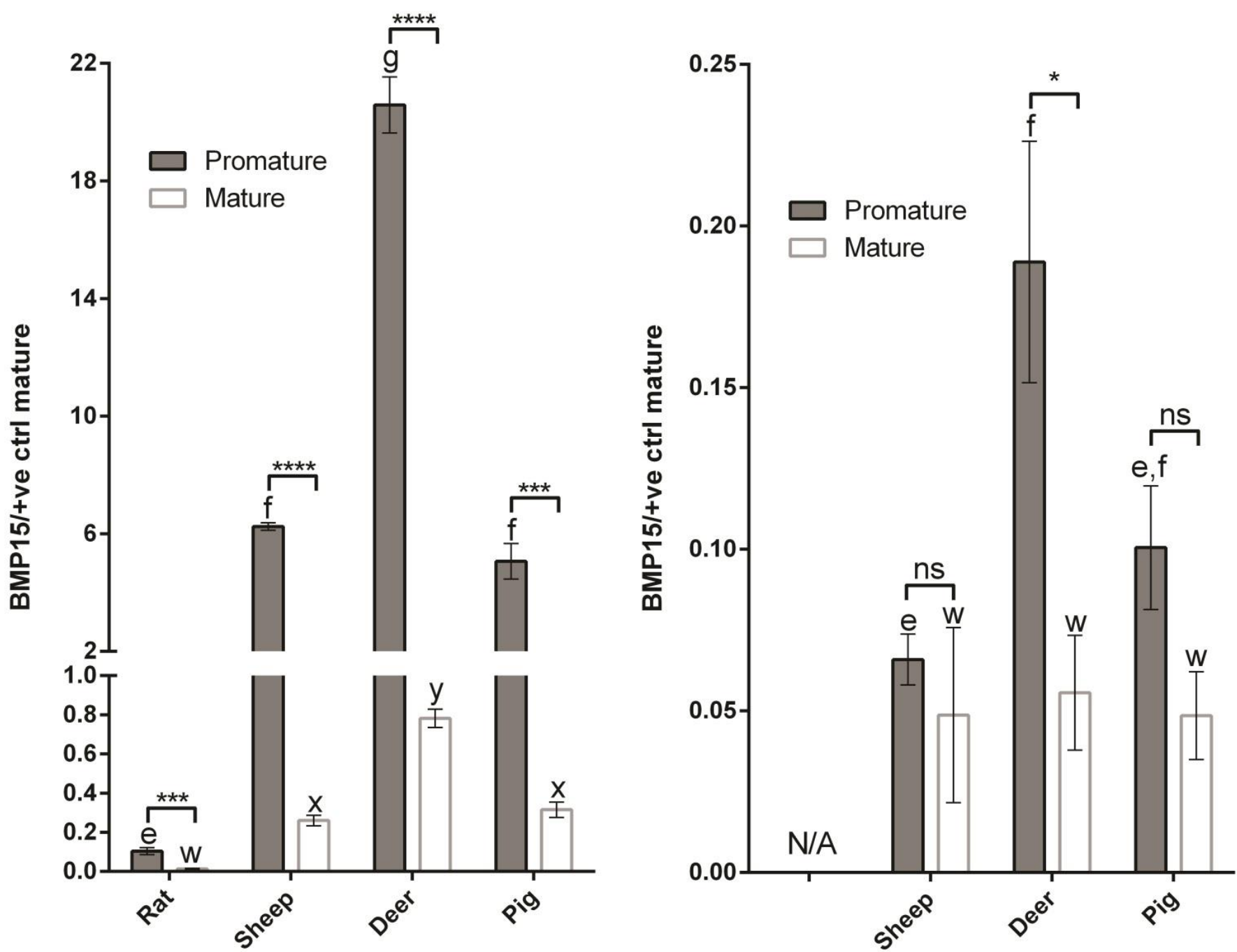

Figure 3.14: Levels of intra-oocyte and oocyte secreted promature and mature BMP15 detected under reducing conditions in each species presented relative to the positive control mature BMP15 band, and adjusted for the number of oocytes used per sample. The experiments in Figures 3.6-3.9 were performed in triplicate and the densities of the bands in the reducing blots were measured. The BMP15 values presented represent the mean ratio between the target-BMP15 band and the mature BMP15 band in the positive control for each blot. Values were adjusted for the number of oocytes used for each sample. The vertical error bars represent \pm SEM. Statistical analysis was performed according to Section 2.1.6. Different letters above the columns for a given protein form indicate a significant difference between species ( $e, f$ and $g$ are used for the promature protein values and $w, x$ and $y$ are used for the mature protein values) $(P \leq 0.05)$. The same letter above two or more columns indicates no significant difference between them. Brackets indicate significant differences between the promature and mature protein values within each species $\left({ }^{*}=\mathrm{P} \leq 0.05, * * *=\mathrm{P} \leq 0.001, * * *=\mathrm{P} \leq 0.0001, \mathrm{~ns}=\right.$ not significant). $\mathrm{N} / \mathrm{A}=$ No BMP15 detected. (A) The levels of promature and mature BMP15 in the oocyte lysate of each species. A broken $y$-axis is used due to the low levels of mature protein observed relative to the promature protein. (B) The levels of promature and mature BMP15 detected in the oocyte conditioned media of each species. 


\subsection{Differences in the ratio of promature:mature BMP15 both within the oocyte and secreted by the oocyte in rats, sheep, pigs and deer}

The ratios of promature to mature BMP15 in the oocyte lysate and oocyte conditioned media samples under reducing conditions were calculated in order to determine if there were differences in the four mammalian species tested (Figure 3.15). In all species, the promature:mature ratio was higher $(P<0.01)$ in the oocyte lysate samples compared to the oocyte conditioned media samples, indicating a marked drop in the levels of promature BMP15 relative to mature BMP15 outside of the oocytes. There were no bands of either protein form detected in the oocyte conditioned media of rats and these results were not included in the analyses.

The promature:mature ratios in oocyte lysate were highest $(P<0.05)$ in sheep and deer (mean promature:mature BMP15 ratios of $\sim 24.5$ and 26.4 , respectively), which were similar, and lower $(P<0.05)$ in rats and pigs (mean promature:mature BMP15 ratios of 7.8 and 16.5, respectively) which were also similar to one another (Figure 3.15).

The promature:mature ratios in oocyte conditioned media were similar between sheep, deer and pigs (mean promature:mature BMP15 ratios of 2.3, 4.7 and 2.3, respectively) whilst levels in rat oocytes were undetectable (Figure 3.15). 


\section{Ratio of promature:mature BMP15}

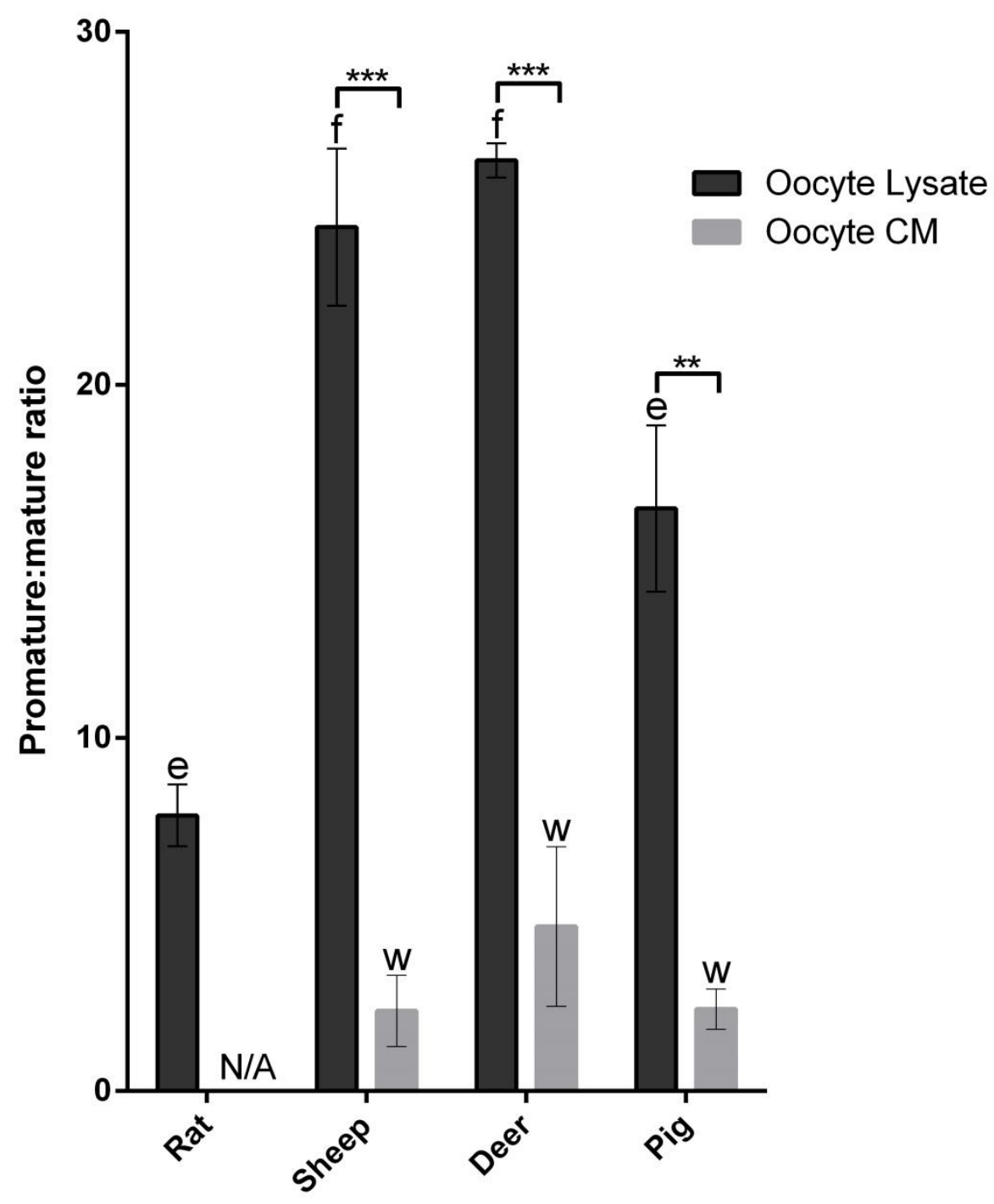

Figure 3.15: Differences in the promature:mature ratio in lysates and conditioned media from in rat, sheep, deer and pig oocytes under reducing conditions. (A) The experiments in Figures 3.6-3.9 were performed in triplicate and the densities of the bands in the reducing blots were measured. The values presented represent the average promature:mature BMP15 ratio for each species in either the oocyte lysate samples or the oocyte conditioned media samples. The vertical error bars represent \pm SEM. Statistical analysis was performed according to Section 2.1.6. Different letters above the columns for a given sample type indicate a significant difference between species ( $e$ and $f$ are used for the oocyte lysate values and $w$ is used for the oocyte conditioned media values) $(P<0.05)$. The same letter above two or more columns indicates no significant difference between them $(P>0.05)$. Brackets indicate significant differences between the oocyte lysate and oocyte conditioned media values within each species except rat where there was no BMP15 detected in the oocyte conditioned media samples. $(* *$ $\mathrm{P}<0.01, * * * \mathrm{P}<0.001)$. N/A= No BMP15 detected. 


\section{Chapter 4 Discussion}

\subsection{Comparisons between two different monoclonal antibodies which were generated to target different areas within the mature BMP15 protein}

Previous studies in our laboratory revealed that the monoclonal Mab61A antibody was able to detect both the promature, and the mature forms of BMP15 on Western blots (see Appendix II; Lin et al., 2012). Due to the finite supply of Mab61A, a new and uncharacterised antibody which was generated to target a similar region of BMP15 mature peptide (Mab61B) and its efficacy was compared with Mab61A. This study provides valuable insight into the variability of detection systems when utilising different monoclonal antibodies to the same target protein. This study undertook a series of experiments comparing the two antibodies under optimised conditions and revealed that the Mab61B antibody had a lower specificity and affinity towards all molecular forms of BMP15, than Mab61A. Moreover, Mab61B was unable to detect promature BMP15 in any of the species tested.

Mab61B required different Western blotting conditions to Mab61A in order to achieve optimum labelling of the mature BMP15 bands, and even under optimised conditions, it showed lower sensitivity than Mab61A in all species except rat (Figures 3.1 and 3.2). These results were surprising because both antibodies target juxtaposed regions of the mature peptide with some overlapping sequences. These regions are located towards the $\mathrm{N}$ terminus of the ovine mature peptide which is believed to be flexible and exposed based on 3D model predictions (Juengel \& McNatty, 2005; McNatty et al., 2007). Furthermore, invivo immunization of sheep using either of the target sequences chemically conjugated to keyhole limpet hemocyan (KLH) elicited a strong immune response and inhibited ovarian function, with the Mab61B target sequence shown to be slightly more effective than the Mab61A target sequence (McNatty et al., 2007). The reason for the differences in affinities between the two antibodies may lie in the fact that both antibodies are monoclonal, and/or that they were each generated in a slightly different manner. Monoclonal antibodies target a specific epitope, and are sensitive to small changes in the structure of the epitope, and factors such as glycosylation may affect their affinity (Lipman et al., 2005). The fact that 
Mab61B was generated against a short synthetic sequence while Mab61A was generated against the entire E.coli-produced mature BMP15 protein may have meant that this generation method provided a better model of the native sequence. It is not uncommon for an antibody generated against a synthetic peptide to have little or no reactivity towards its target protein (Hancock and OReilly, 2005). The fact that the two antibodies have such different binding patterns despite having an 8 amino-acid overlap in their target sites may also be explained by the fact that within a given 15 amino-acid epitope, only $\sim 5$ amino acids contribute the majority of the binding energy (Frank, 2002). Therefore, the remaining 7 amino acids which are unique to the Mab61A target site may play an important role in giving Mab61A a stronger affinity for the protein. The fact that Mab61A could not be fully preadsorbed by the Mab61B target sequence (Peptide B in Figure 3.3) supports this.

Whilst Mab61B was unable to detect promature BMP15 in any of the four species when using a 13.5\% acrylamide gel (Figures 3.1-3.4); the antibody was capable of detecting promature BMP15, albeit with low affinity, in a 7.5\% acrylamide gel. This lower concentration gel allowed for greater separation between the promature protein and similar-sized non-specific target proteins (Figure 3.4). Future experiments required the use of $13.5 \%$ acrylamide gels in order to make comparisons between the mature and promature bands on the same blots. In contrast to Mab61B, Mab61A was able to detect both promature and mature BMP15 in all four species on both $13.5 \%$ and $7.5 \%$ acrylamide gels, and had high affinity for the target proteins and low affinities for non-target proteins. The high cross-affinities of Mab61B to non-target proteins is uncharacteristic of monoclonal antibodies which tend to have high specificity towards their epitope (Lipman et al., 2005). The inability of Mab61B to bind the promature protein on $13.5 \%$ acrylamide gels may be due to non-specific bands of similar molecular weights to the promature band blocking the site on the promature protein where the antibody would bind. Another possible explanation lies in the target sequences for each antibody. The Mab61B target lies directly on the $\mathrm{N}$-terminus of the mature peptide. $\mathrm{N}$-terminal glutamine residues may spontaneously form pyroglutamate under aqueous conditions (Liu et al., 2011). It is possible that the exposed glutamine residue at the $\mathrm{N}$-terminal end of the synthetic peptide sequence used to generate Mab61B may have become a pyroglutamate residue, as has previously been reported in recombinant human BMP15 (Saito et al., 2008). The possible 
use of this pyroglutamate residue to specifically target mature BMP15 and avoid promature BMP15 has been alluded to in previous studies (McMahon, 2007). While further experiments are required to determine if the Mab61B target sequence contains the pyroglutamate residue, it would certainly explain many of the results observed in this study. Explanations aside, it was clear from the comparisons drawn between Mab61A and Mab61B that Mab61A was a superior antibody for the purposes of this study and was the more suitable of the two antibodies for all subsequent experiments.

\subsection{The intra-oocyte and oocyte-secreted molecular forms of BMP15 across a range of mammalian species with different litter sizes (rats, sheep, deer and pigs)}

The Western blots performed using the oocyte lysates of rats, sheep, pigs and deer established that both promature and mature BMP15 was present within oocytes of each species, and are secreted from the oocytes of sheep, deer and pigs (Figures 3.7-3.10). No BMP15 was detected to be secreted from oocytes of rat, possibly due to levels being below the detection limits of the methodology used. While the intra-oocyte and oocyte-secreted forms of BMP15 has been studied previously in rats and sheep using Mab61A under reducing conditions (Lin et al., 2012), the present study provided novel data on two new species that have divergent litter sizes (i.e. pigs and deer). The previous study successfully detected promature and mature BMP15 in sheep oocyte lysates but only mature BMP15 in sheep oocyte conditioned media, and promature BMP15 in rat oocyte lysates (Lin et al., 2012). In the present study following methodology optimisations that allowed for greater detection, a promature BMP15 band was detected in sheep oocyte conditioned media, and a faint mature BMP15 band was detected in oocyte lysates of the rat. This latter observation had previously been reported in another study using an alternative antibody to Mab61A. (Lin et al., 2012). In the present study, the promature and mature bands of the rat and sheep had slightly lower molecular weights than what was reported previously by Lin et al., 2012. This may be due to variations in the loading buffer that was used to dissociate intramolecular disulphide bonds, or differences in the ladder used as the ladder 
used in the current study had more bands in the 10-25 kDa molecular weight range which allowed for a more accurate estimation of the mature band size.

The observations that promature and mature BMP15 are present within the rat oocyte reported in this study and in Lin et al. (2012), and that BMP15 has been shown to stimulate rat granulosa cell proliferation in vitro (Moore et al., 2003; Otsuka et al., 2000) might suggest that BMP15 plays a role in follicular growth and maturation in this species. However, other studies report that only GDF9 is required to enhance granulosa cell proliferation in rats (Lin et al., 2012), and BMP15 knockout studies in mice reveal only a minor loss in fertility (Lin et al., 2012; Yan et al., 2001). Despite BMP15 being present inside the oocytes of rats in the current study, absence of any BMP15 detected outside of the oocyte supports the idea that BMP15 is of little importance in rats. It remains possible that BMP15 was secreted from the oocytes of rats at levels below detection limits, and the low homology between the target sequences of rat and ovine BMP15 for the Mab61A antibody may have contributed to the lack of BMP15 detection in the oocyte conditioned media. However, the study of Lin et al. (2012) used an alternate antibody with a higher homology for rat BMP15 and did not detect BMP15 when using 400 rat oocytes.

As mentioned previously, BMP15 has been viewed as having a lesser importance in mammalian species with high litter sizes, whereby it is thought to have a secondary role to GDF9 (Crawford and McNatty, 2012; Yan et al., 2001). However this study provides novel data on the presence of promature and mature BMP15 protein both within and secreted from the oocytes of pigs, which have a high litter size (14-22 offspring/litter). Moreover, the levels of BMP15 proteins within pig oocytes are similar to that in sheep and deer, which have low litter sizes (1-3 and 1 offspring/litter, respectively) (Figures 3.8-3.10, 3.14). These results are substantiated by reports that BMP15 is actively transcribed during oocyte development in the pig, and that BMP15 may improve oocyte maturation in-vitro, with roles including the prevention of cumulus cell apoptosis (Crawford \& McNatty, 2012; Lin et al., 2014; Paradis et al., 2009; Zhai et al., 2013).

The promature form of BMP15 that is present in the oocyte lysates of all four species, and in the oocyte conditioned media of sheep, deer and pigs appeared to be in an uncleaved form as evidenced by a promature band under reducing conditions. Unprocessed 
promature BMP15 was to be expected within the oocyte as current evidence suggests BMP15 is produced in the same manner as all TGF- $\beta$ proteins, whereby it is produced as preproproteins, with the pre-region removed during translocation into the rough endoplasmic reticulum (RER) (Chang et al., 2002; Dube et al., 1998). Furthermore, high levels of intra-oocyte promature BMP15 has been observed in a number of studies (Guéripel et al., 2006; Lin et al., 2012). More noteworthy was the observation of unprocessed promature BMP15 forms secreted by the oocytes of sheep, deer and pigs. This is because typically only the mature region of the TGF $\beta$ superfamily members is biologically active, and removal of the proregion from the mature peptide usually occurs intracellularly before secretion from the oocyte (Shi et al., 2011; Shimasaki et al., 2004). However, it is not uncommon for a cleaved proregion of TGF $\beta$ superfamily proteins to remain noncovalently associated with their corresponding mature peptides in a latent complex (Shi et al., 2011). This study provides evidence that not all promature BMP15 is cleaved upon secretion from the oocyte in sheep, deer and pigs. While secreted uncleaved promature forms are not common amongst the other TGF $\beta$ superfamily members, a number of studies using recombinant cell lines producing human, sheep or mouse BMP15 have observed uncleaved promature forms in the conditioned media, and one study reported its presence in oocyte secreted media (Hashimoto et al., 2005; McIntosh et al., 2008; McMahon et al., 2008; McNatty et al., 2006; Mester, 2013; Reader et al., 2011).

The presence of unprocessed promature BMP15 in the oocyte secreted media of sheep, deer and pigs suggests that it may have a biological function. Extracellularly, the proregions of TGF- $\beta$ superfamily proteins are reported to act as chaperones for delivering the mature ligand to their target cells. The hypothesised mechanism of action is that the proregion interacts with the extracellular matrix and also with potential proregion targets such as heparin which are expressed differentiationally on the extracellular domain of granulosa cells and cumulus cells, and mediate the presentation of the mature peptide to the surface receptors (Harrison et al., 2011; Mottershead et al., 2015; Sengle et al., 2008). Therefore, the promature BMP15 secreted from the oocytes of sheep, pigs and deer may allow each species to have additional extracellular mechanisms for species-specific regulation of BMP15 signalling. Few studies have investigated the biological activity of uncleaved promature BMP15, however one such study demonstrated that it is biologically active 
(Mester, 2013), and it may be more effective at increasing bovine embryo development than mature BMP15, mature GDF9, or promature GDF9 (Sudiman et al., 2014). Furthermore, a heterodimeric complex between promature BMP15 and promature GDF9 (named pro-cumulin) has been demonstrated to elicit potent bioactivity on ovine granulosa cells and oocytes, as well as improve oocyte quality and embryo development (Mottershead et al., 2015). It should be noted that the model presented in the study by Mottershead et al., 2015 believed the promature proteins in the pro-cumulin complex existed in a semi-processed state, where the proregions were non-covalently associated with the mature regions. However, this model was based on the assumption that BMP15 is processed in the same manner as other TGF- $\beta$ proteins, and therefore all the secreted promature BMP15 is in a semi-processed state. While the results from this current study do not rule out the possibility that some secreted promature protein is secreted in a semiprocessed state, there is strong evidence that a significant portion is in an unprocessed form, which was a possibility not taken into account in the Mottershead et al., 2015 study.

In all four species included in this study, there was no evidence that mature or promature BMP15/BMP15 homodimers or BMP15/GDF9 heterodimers exist either within oocytes or as forms secreted from the oocyte (Figures 3.7-3.11). BMP15 and GDF9 lack the seventh cysteine residue that forms the disulphide bridge between two monomer subunits, presumably allowing for more flexible interactions between the monomer units (Chang et al., 2002; Shimasaki et al., 2004a). While a large number of studies have identified homodimers and heterodimers in recombinantly-produced forms of BMP15 and GDF9 (Hashimoto et al., 2005; Liao et al., 2003; Liao, Moore, \& Shimasaki, 2004; McIntosh et al., 2008; Mottershead et al., 2015; Peng et al., 2013a; Shimasaki, Moore, Otsuka, \& Erickson, 2004b), there is no evidence to suggest these forms exist in vivo. There are a range of possible explanations for the absence of any dimer detection when using native oocyte produced proteins in this study. It is possible that dimers were present but below the threshold of detectability for Western blotting. To minimise this possibility, exposure times for Western blots were lengthened to increase the detectability of faint bands, yet still no dimer bands were detected. Another possibility is that the Mab61A antibody used had a lower affinity for dimeric forms of BMP15, however this is unlikely to be the case for mature BMP15 dimers as this antibody detected a mature homodimer in the recombinant HEK-293 
produced pig BMP15 (positive control) after crosslinking in this study (Figures 3.7-3.10). It remains possible that the antibody target site in promature dimeric BMP15 forms are blocked because no promature dimers were detected, even using recombinant protein samples. However, this is unlikely due to crosslinked proteins being exposed to reducing conditions that should linearize the proteins and thus expose these sites. Finally, it is possible that native dimers are not stable under the collection conditions. Despite all these limitations, the absence of any BMP15 dimers under the conditions used in this study, together with the absence of any other evidence of native BMP15 dimer formation in the literature poses an interesting question about the differences between recombinant and oocyte produced (native) forms of BMP15 in regards to production and stability of BMP15 dimers.

This study also elucidated that at least some of the promature and mature BMP15 forms exist as monomers although this was species-specific. Whilst monomers existed in the oocyte lysates of all four species, secreted monomer forms were only detected pigs and deer as demonstrated by the presence of monomer bands following crosslinking (Figures 3.7-3.10). Moreover, whilst mature BMP15 monomers were detected in secretions from oocytes of sheep, promature monomers were not. Possible monomer forms of BMP15 have been observed following crosslinking using recombinantly-produced ovine and mouse BMP15 in previous studies (Edwards et al., 2008; McIntosh et al., 2008). As of yet, it remains unknown whether these native monomers are biologically active. Current evidence suggests that dimerization of BMP15 either with itself or with GDF9 is required at the level of the receptor for receptor activation and down-stream effects on granulosa cells. Moreover, it is reported that receptor complexes that include heterodimers elicit a stronger biological effect than homodimers in a number of species (Lin et al., 2006; Mclntosh et al., 2008; Mottershead et al., 2015; Peng et al., 2013b).

Whilst recombinant forms of BMP15, and GDF9, may not be identical to those observed in oocyte produced samples, they are valuable in determining why these differences may occur. From extrapolations of recombinantly-produced BMP15 and GDF9, it has been suggested that BMP15 and GDF9 heterodimers may spontaneously form either inside the oocyte, or in the extracellular space (Mottershead et al., 2015). The reasoning for this is that TGF- $\beta$ monomers are highly hydrated at the dimerization interface, thus any 
homodimers initially formed during protein production have limited stability. While other TGF- $\beta$ superfamily members overcome this relative instability by forming a disulphide bond between the two monomers, the inability for BMP15 or GDF9 to form these bonds results in a mixture of dimers that are constantly exchanging monomer units (Mottershead et al., 2015). While the model presented by Mottershead et al., 2015, suggested an equilibrium between BMP15 and GDF9 homodimers and heterodimers both inside and outside the oocyte, the results of this study suggest that the equilibrium may also include monomer units of either protein, and if dimers are forming, they may be transient in nature which may explain why no dimers were detected in this study. However, while this study allows for this possibility, it does not provide any direct evidence for transient dimer formation and further research into this possibility is required. If, with further research, the monomers detected do turn out to play a role in possible dimer formation and/or have bioactivity, then differences in the presence of these monomer forms may be speciesspecific, and may influence litter size through differences in the bioavailability of monomers for potential dimer formation. This would have implications for the use of recombinant forms of BMP15 and GDF9 in protocols for in vitro maturation (IVM) of cumulus cell-oocyte complexes to increase oocyte quality and embryological outcome. The effective use of these growth factors in IVM may be heavily dependent on providing optimal extracellular conditions for weak transient dimers to form, and provision of the correct amount of their promature and mature monomer forms.

Covalent, and possibly non-covalent, multimers were observed in the oocyte lysates of all four species, and in the oocyte conditioned media of deer, pigs and sheep as indicated by the presence of prominent bands in the 150-250 kDa region under crosslinking and/or nonreducing conditions (Figures 3.7-3.10). Where multimers were observed, similar molecular weight bands were observed in the anti-GDF9 labelled samples suggesting the presence of BMP15/GDF9 multimers. The broad multimer band on the Western blot suggests a range of different multimeric compounds and has been observed in previous studies using recombinant proteins (Edwards et al., 2008; McIntosh et al., 2008; Mester, 2013; Reader et al., 2011). It was proposed that the multimers contain combinations of proregion and mature proteins of BMP15 and GDF9, and that the proregion may be important in multimer formation; however the precise components of the multimers remained largely undefined. 
It also remains a possibility that the multimer bands are simply an artefact caused by the Western blot methodology.

The molecular weights of the promature and mature bands in this study were generally less than those observed in previous studies using recombinant BMP15 (Hashimoto et al., 2005; McIntosh et al., 2008; McMahon et al., 2008; Reader et al., 2011). However, recombinant forms of sheep, deer and pig BMP15 used in the current study had similar molecular weights to the corresponding native forms, and thus these discrepancies are likely to be due to methodology differences or differences between molecular weight ladders. Interestingly, the molecular weights of the promature and mature bands for each species were $\sim 5$ and $\sim 2 \mathrm{kDa}$ heavier than the expected calculated weight based on the amino-acid sequence alone ("ExPASy - Compute pl/Mw tool," 2016). This may be due to the proteins being glycosylated and/or phosphorylated as promature and mature BMP15 has five and two potential glycosylation sites respectively, and both may be phosphorylated (Dube et al., 1998; McMahon et al., 2008). Protein glycosylation may explain the band laddering effect observed in sheep, deer and pig oocyte lysate samples, and the double bands present in oocyte conditioned media samples in this study. It has been reported that glycosylation and/or phosphorylation may alter the bioactivity of BMP15 proteins (McIntosh et al., 2008; McMahon et al., 2008). Thus, the prominent laddering observed in the species with a low litter size (sheep and deer) compared to the faint or no laddering observed in the species with high litter size (pig and rat) may indicate differential post-transcriptional processing of BMP15 in high and low litter size species, and thus differences in the bioactivity of the BMP15 present. In this present study, chemical deglycosylation experiments were attempted using a modified version of a previously-published protocol (Horvath et al., 1989) however, the protein lost during the deglycosylation process together with only nanogram quantities of starting material resulted in no identifiable bands (data not shown). In future studies, an enzymatic deglycosylation of the BMP15 may be more suitable.

When assessing protein forms on a Western blot, it is often difficult to assess which bands are due to specific binding of the antibody. Monoclonal antibodies are designed to be highly specific towards their target sequence, however it is possible for non-specific target sites to exist (Frank, 2002; Holmseth et al., 2012). Preadsorption experiments were performed using E.coli-produced ovine BMP15 which led to a complete loss of bands at all 
of the expected BMP15 sizes but also in additional bands. The disappearance of bands following preabsorption has traditionally been used to test specific binding of polyclonal antibodies. However in this case, Mab61A was a monoclonal antibody, and preadsorbtion was performed using the same target antigen (E. coli produced mature ovine BMP15) that it was screened against during production. Therefore, unlike with polyclonal antibodies, all bands, regardless of specificity, would be expected to disappear (Holmseth et al., 2012; Saper, 2009). Furthermore, stripping of Western blots leads to protein loss (Appendix IV), and thus faint bands in original blots may have been lost through this process.

\subsection{The relative amounts of promature and mature BMP15 across a range of mammalian species with different litter sizes (rats, sheep, deer and pigs)}

Of the two different methods for normalising the relative amounts of BMP15 illustrated in Figures 3.13 and 3.14 , the method whereby values were presented relative to the number of oocytes per sample and normalised using the mature BMP15 band in the positive control (Figure 3.14) was deemed most appropriate for detailed discussion, and the reasons for this are described later in this discussion section.

There was markedly more promature and mature BMP15 observed in the oocyte lysates of deer compared to the other three species (Figure 3.14 A). This indicates that that BMP15 may play a greater role in follicular growth and maturation in deer than in the other species tested. There were moderate levels of both molecular forms of BMP15 in the sheep, but very low levels in the rat. These results align well with previous research suggesting that BMP15 is essential for low ovulation rate species, and may be less important in some high ovulation rate species (Galloway et al., 2000; Hanrahan et al., 2004; Yan et al., 2001). The BMP15 levels within the pig oocyte lysate did not conform to this trend, as the levels of each protein form were similar to sheep. It was previously reported that both BMP15 and GDF9 mRNA expression levels per oocyte are high in the pig compared to sheep, deer or rats (Crawford \& McNatty, 2012). The BMP15 mRNA expression of pigs was $\sim 4-6$ fold higher than sheep or deer, and 18 fold higher than rats. In this current study, the protein levels 
of BMP15 were lower than deer and similar to sheep. This suggests that species differences in the post-transcriptional processing of BMP15 occur, and it raises the possibility that the GDF9:BMP15 protein ratio in pigs may be more in-line with what is expected in a high ovulatory species. Further investigation is required to determine if this is the case. Regardless, these results clearly indicate that BMP15 protein levels within oocytes alone do not correlate with litter size for all species.

The secreted forms of promature BMP15 were higher in deer than sheep and rats, but similar to that in pigs (Figure 3.14 B). Unlike the oocyte lysate samples, the levels of mature protein in the oocyte conditioned media samples were similar between deer, sheep and pigs. Whilst this suggests that the levels of mature BMP15 produced within an oocyte do not necessarily correlate with that secreted, it may be due to a lack of sensitivity of the Western blotting technique.

The promature:mature BMP15 ratios in the oocyte lysates were similar in the two low ovulation rate species (sheep and deer), and similar in the two high ovulation species (pig and rat), with the ratio being markedly higher in the low ovulation species (Figure 3.15). The lower ratio in the species with large litter sizes does not conform well with a number of studies whereby mutations that lowered promature BMP15 processing (i.e. increased the promature:mature ratio) led to higher fertility (Hanrahan et al., 2004; Hashimoto et al., 2005; Shimasaki et al., 2004). These anomalies may be partially explained by the fact that both pigs and rats have mutations in the RXXR cleavage region resulting in unique residues in the XX portion of the furin-like protease cleavage site while sheep and deer both share the RRAR cleavage sequence. This may results in differences in processing efficiencies of the furin-like proteases involved in the cleavage process in rats and pigs, compared to sheep and deer.

There was no differences between the ratios of secreted promature:mature forms in the sheep, deer and pig, with the ratio declining markedly in each species compared to what was observed within the oocyte (Figure 3.15). The decline in the promature:mature ratio in the forms secreted outside of the oocyte suggests that the promature form may be less readily secreted than the processed mature form, which has been suggested previously (Shimasaki et al., 2004), or that the processing of the promature protein occurs more 
rapidly outside of the cell. Another possibility is that extracellular protease activity under in-vitro conditions led to increased promature processing. While protease inhibitors were added after the incubation of the oocytes, protease activity in the conditioned media may still have occurred during the incubation period. This possibility is supported by studys that revealed that only the unprocessed promature form was present in follicular fluid of sheep (McNatty et al., 2006), while processed and unprocessed forms were detected in media from in-vitro culturing of sheep oocytes (Lin et al., 2012, this study).

As mentioned earlier, two different methods for normalizing BMP15 results were explored in this study which are displayed in Figures 3.13 and 3.14, and described in Section 2.1.6. The principle behind normalizing the bands against $\beta$-actin levels was that $\beta$-actin acts as a house-keeping protein (HKP) in oocytes, and as such, it is assumed that $\beta$-actin levels are relative to total protein levels (Ferguson et al., 2005). Furthermore, $\beta$-actin is a highly conserved protein (Gunning et al., 2015; Rubenstein, 1990), and as such, the same antibody could be used to detect $\beta$-actin in each species in this study. However, a number of deficiencies were identified using this method in this study. Firstly, $\beta$-actin, like many HKPs has been shown to have inconsistent translation efficiency across tissue types and sample conditions (Ferguson et al., 2005; Li \& Shen, 2013). Secondly, $\beta$-actin was highly abundant in the oocyte lysate samples therefore shorter exposure times were used to avoid image saturation, common for HKP in cell lysate samples (Taylor \& Posch, 2014). These shorter exposure times will decrease the sensitivity of this method. Thirdly, $\beta$-actin is not secreted and thus absent in the conditioned media samples. Therefore, values from the corresponding oocyte lysate samples were extrapolated to corresponding media samples however transfer and loading efficiencies between the sample types were unknown. Finally and most importantly, there were species differences in the ease of denuding oocytes, and as such, oocytes from pigs were fully denuded of cumulus cells, whilst the others were partially denuded. Therefore, $\beta$-actin levels in oocyte samples from rats, sheep and deer also contained cumulus cell-derived $\beta$-actin. This would result in higher $\beta$-actin levels in these species and result in low normalised values of BMP15, as observed Figure 3.13.

An alternative approach used was to express the density result per oocyte, and normalise against the density value of the mature BMP15 band in the positive control that was present in every blot. This also accounted for inter-blot variation due to different transfer 
and labelling efficiencies between blots. This approach also had several limitations. It did not control for differences in transfer and loading efficiency across all of the lanes present in each blot. It also relies on the consistent loading of the positive control between blots. Despite these limitations, it appeared to provide a way for semi-quantifying and comparing the amount of BMP15 within, and secreted from, oocytes in the different species. 


\subsection{Study Conclusions}

This study clearly demonstrated species differences in the molecular forms of BMP15. Deer, sheep and rats conform to the hypothesis that BMP15 levels are inversely related to litter size. That is, the intra-oocyte levels, and largely the secreted levels, of BMP15 ranked from highest to lowest were that in deer, sheep and rats, respectively. Pigs were an exception whereby their oocytes contained similar levels of BMP15 to sheep. As expected, secreted forms of rat BMP15 were negligible. These results imply that BMP15 may play a role in follicular growth and maturation in some high ovulation rate species, as well as low ovulation rate species. Overall, these results suggest that levels of BMP15 are not the sole determinant of litter size.

The current study failed to detect BMP15/BMP15 homodimers or BMP15/GDF9 heterodimers in any of samples collected from the four species. Monomers of promature and mature BMP15 were detected, as well as possible multimeric forms. The detection of dimers in recombinantly-produced BMP15 suggests that there are differences between recombinantly-produced and native forms of BMP15. Therefore, conclusions made from studies using recombinant BMP15 may not necessarily provide a fair representation of the forms, and thus biological activity of BMP15, in vivo.

This study showed that the monoclonal antibody Mab61A was suitable for the detection of the different molecular forms of BMP15 in numerous species. Comparisons between two monoclonal antibodies highlighted the variability in affinities that can occur when generated using different methods (whole protein versus short peptide sequence), even when the target sequences overlap. This study emphasises the importance of testing multiple antibodies before making conclusions about protein forms present within Western blot assays. 


\subsection{Future Directions}

To fully appreciate the findings of this study, it would be useful to repeat the Western blot experiments and immuno-label for GDF9. By keeping experimental conditions similar but optimising for oocyte number in regards to GDF9 production, it would be possible to compare the relative amounts of each protein, and determine how the GDF9:BMP15 protein ratios relate to a previous study that reported Gdf9:Bmp15 mRNA ratios in the same species (Crawford \& McNatty, 2012). Of particular interest would be whether the GDF9:BMP15 protein ratio is similar to the low Gdf9:Bmp15 mRNA ratio observed in pigs.

It would be useful to collect larger sample sizes and concentrate the samples using immunoprecipitation. The concentrated levels of BMP15 in the samples, particularly in the oocyte conditioned media, would allow for better detection of lowly-translated proteins. It may also allow for the detection of dimer forms if present, or more conclusively prove their absence. Furthermore, the development of purified mutated recombinant forms of covalently bound promature and mature BMP15/BMP15 homodimers, and BMP15/GDF9 heterodimers for each species would confirm whether the Mab61A antibody is capable of detecting these dimers. This would support the results from this study by determining whether or not the absence of dimer forms was due to the limitations of the antibody. Also, further investigation of the multimer complexes using $7.5 \%$ acrylamide gels and a range of different antibodies may provide insight into the different non-monomer protein forms present.

Posttranslational modifications such as glycosylation and phosphorylation have often been suspected of having a role in BMP15 bioactivity. It was hypothesised that the laddering of bands in several blots may be due to posttranslational modifications of the protein. While chemical deglycosylation proved unsuccessful and led to a complete loss of protein, deglycosylation using an enzyme such as PNGase F may be more successful. This would provide insight into whether glycosylation levels vary between species, and between recombinant and native oocyte-produced forms of BMP15. Finally, a similar set of experiments using a greater range of mammalian species that differ in litter size may help determine whether the general hypothesis that BMP15 levels are inversely related to litter size is valid. 


\section{Chapter 5: Literature Cited}

Aaltonen, J., Laitinen, M.P., Vuojolainen, K., Jaatinen, R., Horelli-Kuitunen, N., Seppä, L., Louhio, H., Tuuri, T., Sjöberg, J., Bützow, R., Hovata, O., Dale, L., Ritvos, O., 1999. Human growth differentiation factor 9 (GDF-9) and its novel homolog GDF-9B are expressed in oocytes during early folliculogenesis. J. Clin. Endocrinol. Metab. 84, 2744-2750. doi:10.1210/jcem.84.8.5921

Al-Musawi, S.L., Walton, K.L., Heath, D., Simpson, C.M., Harrison, C.A., 2013. Species differences in the expression and activity of bone morphogenetic protein 15 . Endocrinology 154, 888-899. doi:10.1210/en.2012-2015

Bodensteiner, K.J., McNatty, K.P., Clay, C.M., Moeller, C.L., Sawyer, H.R., 2000. Expression of growth and differentiation factor-9 in the ovaries of fetal sheep homozygous or heterozygous for the inverdale prolificacy gene (FecX(I)). Biol. Reprod. 62, 14791485.

Brunner, A.M., Lioubin, M.N., Marquardt, H., Malacko, A.R., Wang, W.C., Shapiro, R.A., Neubauer, M., Cook, J., Madisen, L., Purchio, A.F., 1992. Site-directed mutagenesis of glycosylation sites in the transforming growth factor-beta 1 (TGF beta 1 ) and TGF beta 2 (414) precursors and of cysteine residues within mature TGF beta 1: effects on secretion and bioactivity. Mol. Endocrinol. 6, 1691-1700. doi:10.1210/mend.6.10.1448117

Chang, H., Brown, C.W., Matzuk, M.M., 2002. Genetic analysis of the mammalian transforming growth factor-beta superfamily. Endocr. Rev. 23, 787-823. doi:10.1210/er.2002-0003

Cheifetz, S., Weatherbee, J.A., Tsang, M.L.-S., Anderson, J.K., Mole, J.E., Lucas, R., Massagué, J., 1987. The transforming growth factor- $\beta$ system, a complex pattern of cross-reactive ligands and receptors. Cell 48, 409-415. doi:10.1016/00928674(87)90192-9 
Constam, D.B., Robertson, E.J., 1999. Regulation of Bone Morphogenetic Protein Activity by Pro Domains and Proprotein Convertases. J Cell Biol 144, 139-149.

Crawford, J.L., McNatty, K.P., 2012. The ratio of growth differentiation factor 9: bone morphogenetic protein 15 mRNA expression is tightly co-regulated and differs between species over a wide range of ovulation rates. Mol. Cell. Endocrinol. 348, 339-343. doi:10.1016/j.mce.2011.09.033

de Caestecker, M., 2004. The transforming growth factor-beta superfamily of receptors. Cytokine Growth Factor Rev. 15, 1-11.

Di Pasquale, E., Beck-Peccoz, P., Persani, L., 2004. Hypergonadotropic Ovarian Failure Associated with an Inherited Mutation of Human Bone Morphogenetic Protein-15 (BMP15) Gene. Am J Hum Genet 75, 106-111.

Dong, J., Albertini, D.F., Nishimori, K., Kumar, T.R., Lu, N., Matzuk, M.M., 1996. Growth differentiation factor-9 is required during early ovarian folliculogenesis. Nature 383, 531-535. doi:10.1038/383531a0

Dragovic, R.A., Ritter, L.J., Schulz, S.J., Amato, F., Thompson, J.G., Armstrong, D.T., Gilchrist, R.B., 2007. Oocyte-secreted factor activation of SMAD $2 / 3$ signaling enables initiation of mouse cumulus cell expansion. Biol. Reprod. 76, 848-857. doi:10.1095/biolreprod.106.057471

Dube, J.L., Wang, P., Elvin, J., Lyons, K.M., Celeste, A.J., Matzuk, M.M., 1998. The Bone Morphogenetic Protein 15 Gene Is X-Linked and Expressed in Oocytes. Molecular Endocrinology 12, 1809-1817. doi:10.1210/mend.12.12.0206

Edwards, S.J., Reader, K.L., Lun, S., Western, A., Lawrence, S., McNatty, K.P., Juengel, J.L., 2008. The cooperative effect of growth and differentiation factor-9 and bone morphogenetic protein (BMP)-15 on granulosa cell function is modulated primarily through BMP receptor II. Endocrinology 149, 1026-1030. doi:10.1210/en.20071328 
ExPASy - Compute pl/Mw tool [WWW Document], 2016. . ExPASy Bioinformatics Resource Portal. URL http://web.expasy.org/compute_pi//(accessed 15.1.16).

Fabre, S., Pierre, A., Mulsant, P., Bodin, L., Di Pasquale, E., Persani, L., Monget, P., Monniaux, D., 2006. Regulation of ovulation rate in mammals: contribution of sheep genetic models. Reprod Biol Endocrinol 4, 20. doi:10.1186/1477-7827-4-20

Fairlie, W.D., Zhang, H.P., Wu, W.M., Pankhurst, S.L., Bauskin, A.R., Russell, P.K., Brown, P.K., Breit, S.N., 2001. The propeptide of the transforming growth factor-beta superfamily member, macrophage inhibitory cytokine-1 (MIC-1), is a multifunctional domain that can facilitate protein folding and secretion. J. Biol. Chem. 276, 16911-16918. doi:10.1074/jbc.M010000200

Fares, F., 2006. The role of O-linked and N-linked oligosaccharides on the structure-function of glycoprotein hormones: development of agonists and antagonists. Biochim. Biophys. Acta 1760, 560-567. doi:10.1016/j.bbagen.2005.12.022

Fenwick, M.A., Mora, J.M., Mansour, Y.T., Baithun, C., Franks, S., Hardy, K., 2013. Investigations of TGF- $\beta$ signaling in preantral follicles of female mice reveal differential roles for bone morphogenetic protein 15. Endocrinology 154, 34233436. doi:10.1210/en.2012-2251

Ferguson, R.E., Carroll, H.P., Harris, A., Maher, E.R., Selby, P.J., Banks, R.E., 2005. Housekeeping proteins: A preliminary study illustrating some limitations as useful references in protein expression studies. Proteomics 5, 566-571. doi:10.1002/pmic.200400941

Fitzpatrick, S.L., Sindoni, D.M., Shughrue, P.J., Lane, M.V., Merchenthaler, I.J., Frail, D.E., 1998. Expression of growth differentiation factor-9 messenger ribonucleic acid in ovarian and nonovarian rodent and human tissues. Endocrinology 139, 2571-2578. doi:10.1210/endo.139.5.6014

Frank, S.A., 2002. Immunology and Evolution of Infectious Disease. Princeton University Press, Princeton (NJ). 
Galloway, S.M., McNatty, K.P., Cambridge, L.M., Laitinen, M.P., Juengel, J.L., Jokiranta, T.S., McLaren, R.J., Luiro, K., Dodds, K.G., Montgomery, G.W., Beattie, A.E., Davis, G.H., Ritvos, O., 2000. Mutations in an oocyte-derived growth factor gene (BMP15) cause increased ovulation rate and infertility in a dosage-sensitive manner. Nat. Genet. 25, 279-283. doi:10.1038/77033

Gilchrist, R.B., 2011. Recent insights into oocyte-follicle cell interactions provide opportunities for the development of new approaches to in vitro maturation. Reprod. Fertil. Dev. 23, 23-31. doi:10.1071/RD10225

Gilchrist, R.B., Lane, M., Thompson, J.G., 2008. Oocyte-secreted factors: regulators of cumulus cell function and oocyte quality. Hum. Reprod. Update 14, 159-177. doi:10.1093/humupd/dmm040

Guéripel, X., Brun, V., Gougeon, A., 2006. Oocyte Bone Morphogenetic Protein 15, but not Growth Differentiation Factor 9, Is Increased During Gonadotropin-Induced Follicular Development in the Immature Mouse and Is Associated with Cumulus Oophorus Expansion. Biol Reprod 75, 836-843. doi:10.1095/biolreprod.106.055574

Gunning, P.W., Ghoshdastider, U., Whitaker, S., Popp, D., Robinson, R.C., 2015. The evolution of compositionally and functionally distinct actin filaments. J Cell Sci 128, 2009-2019. doi:10.1242/jcs.165563

Guo, J., Wu, G., 2012. The signaling and functions of heterodimeric bone morphogenetic proteins. Cytokine \& Growth Factor Reviews 23, 61-67. doi:10.1016/j.cytogfr.2012.02.001

Hancock, D., OReilly, N., 2005. Synthetic Peptides as Antigens for Antibody Production, in: Burns, R. (Ed.), Immunochemical Protocols, Methods In Molecular Biology ${ }^{\mathrm{TM}}$. Humana Press, pp. 13-25.

Hang, Q., Zhou, Y., Hou, S., Zhang, D., Yang, X., Chen, J., Ben, Z., Cheng, C., Shen, A., 2014. Asparagine-linked glycosylation of bone morphogenetic protein-2 is required for 
secretion and osteoblast differentiation. Glycobiology 24, 292-304. doi:10.1093/glycob/cwt110

Hanrahan, J.P., Gregan, S.M., Mulsant, P., Mullen, M., Davis, G.H., Powell, R., Galloway, S.M., 2004. Mutations in the genes for oocyte-derived growth factors GDF9 and BMP15 are associated with both increased ovulation rate and sterility in Cambridge and Belclare sheep (Ovis aries). Biol. Reprod. 70, 900-909. doi:10.1095/biolreprod.103.023093

Hariharan, R., Pillai, M.R., 2008. Structure-function relationship of inhibitory Smads: Structural flexibility contributes to functional divergence. Proteins $71,1853-1862$. doi:10.1002/prot.21869

Harrison, C.A., Al-Musawi, S.L., Walton, K.L., 2011. Prodomains regulate the synthesis, extracellular localisation and activity of TGF- $\beta$ superfamily ligands. Growth Factors 29, 174-186. doi:10.3109/08977194.2011.608666

Hashimoto, O., Moore, R.K., Shimasaki, S., 2005. Posttranslational processing of mouse and human BMP-15: Potential implication in the determination of ovulation quota. Proc Natl Acad Sci U S A 102, 5426-5431. doi:10.1073/pnas.0409533102

Hauburger, A., von Einem, S., Schwaerzer, G.K., Buttstedt, A., Zebisch, M., Schräml, M., Hortschansky, P., Knaus, P., Schwarz, E., 2009. The pro-form of BMP-2 interferes with BMP-2 signalling by competing with BMP-2 for IA receptor binding. FEBS Journal 276, 6386-6398. doi:10.1111/j.1742-4658.2009.07361.x

Hayashi, M., McGee, E.A., Min, G., Klein, C., Rose, U.M., Duin, M. van, Hsueh, A.J.W., 1999. Recombinant Growth Differentiation Factor-9 (GDF-9) Enhances Growth and Differentiation of Cultured Early Ovarian Follicles. Endocrinology 140, 1236-1244. doi:10.1210/endo.140.3.6548

Hobbs, S., Jitrapakdee, S., Wallace, J.C., 1998. Development of a bicistronic vector driven by the human polypeptide chain elongation factor 1alpha promoter for creation of 
stable mammalian cell lines that express very high levels of recombinant proteins. Biochem. Biophys. Res. Commun. 252, 368-372. doi:10.1006/bbrc.1998.9646

Hogan, B.L., 1996. Bone morphogenetic proteins: multifunctional regulators of vertebrate development. Genes Dev. 10, 1580-1594. doi:10.1101/gad.10.13.1580

Holmseth, S., Zhou, Y., Follin-Arbelet, V.V., Lehre, K.P., Bergles, D.E., Danbolt, N.C., 2012. Specificity Controls for Immunocytochemistry. J Histochem Cytochem 60, 174-187. doi:10.1369/0022155411434828

Horvath, E., Edwards, A.M., Bell, J.C., Braun, P.E., 1989. Chemical deglycosylation on a micro-scale of membrane glycoproteins with retention of phosphoryl-protein linkages. J. Neurosci. Res. 24, 398-401. doi:10.1002/jnr.490240309

Incerti, B., Dong, J., Borsani, G., Matzuk, M.M., 1994. Structure of the mouse growth/differentiation factor 9 gene. Biochim. Biophys. Acta 1222, 125-128.

Juengel, J.L., Bodensteiner, K.J., Heath, D.A., Hudson, N.L., Moeller, C.L., Smith, P., Galloway, S.M., Davis, G.H., Sawyer, H.R., McNatty, K.P., 2004. Physiology of GDF9 and BMP15 signalling molecules. Animal Reproduction Science, Research and Practice III. 15th International Congress on Animal Reproduction 82-83, 447-460. doi:10.1016/j.anireprosci.2004.04.021

Juengel, J.L., Hudson, N.L., Berg, M., Hamel, K., Smith, P., Lawrence, S.B., Whiting, L., McNatty, K.P., 2009. Effects of active immunization against growth differentiation factor 9 and/or bone morphogenetic protein 15 on ovarian function in cattle. Reproduction 138, 107-114. doi:10.1530/REP-09-0009

Juengel, J.L., Hudson, N.L., Heath, D.A., Smith, P., Reader, K.L., Lawrence, S.B., O'Connell, A.R., Laitinen, M.P.E., Cranfield, M., Groome, N.P., Ritvos, O., McNatty, K.P., 2002. Growth Differentiation Factor 9 and Bone Morphogenetic Protein 15 Are Essential for Ovarian Follicular Development in Sheep. Biol Reprod 67, 1777-1789. doi:10.1095/biolreprod.102.007146 
Juengel, J.L., Hudson, N.L., Whiting, L., McNatty, K.P., 2004. Effects of immunization against bone morphogenetic protein 15 and growth differentiation factor 9 on ovulation rate, fertilization, and pregnancy in ewes. Biol. Reprod. 70, 557-561. doi:10.1095/biolreprod.103.023333

Juengel, J.L., McNatty, K.P., 2005. The role of proteins of the transforming growth factorbeta superfamily in the intraovarian regulation of follicular development. Hum. Reprod. Update 11, 143-160. doi:10.1093/humupd/dmh061

Knight, P.G., Glister, C., 2006. TGF-beta superfamily members and ovarian follicle development. Reproduction 132, 191-206. doi:10.1530/rep.1.01074

Kusakabe, M., Cheong, P.-L., Nikfar, R., McLennan, I.S., Koishi, K., 2008. The structure of the TGF-beta latency associated peptide region determines the ability of the proprotein convertase furin to cleave TGF-betas. J. Cell. Biochem. 103, 311-320. doi:10.1002/jcb.21407

Laissue, P., Christin-Maitre, S., Touraine, P., Kuttenn, F., Ritvos, O., Aittomaki, K., Bourcigaux, N., Jacquesson, L., Bouchard, P., Frydman, R., Dewailly, D., Reyss, A.-C., Jeffery, L., Bachelot, A., Massin, N., Fellous, M., Veitia, R.A., 2006. Mutations and sequence variants in GDF9 and BMP15 in patients with premature ovarian failure. Eur. J. Endocrinol. 154, 739-744. doi:10.1530/eje.1.02135

Laitinen, M., Vuojolainen, K., Jaatinen, R., Ketola, I., Aaltonen, J., Lehtonen, E., Heikinheimo, M., Ritvos, O., 1998. A novel growth differentiation factor-9 (GDF-9) related factor is co-expressed with GDF-9 in mouse oocytes during folliculogenesis. Mechanisms of Development 78, 135-140. doi:10.1016/S0925-4773(98)00161-0

Liao, W.X., Moore, R.K., Otsuka, F., Shimasaki, S., 2003. Effect of intracellular interactions on the processing and secretion of bone morphogenetic protein-15 (BMP-15) and growth and differentiation factor-9. Implication of the aberrant ovarian phenotype of BMP-15 mutant sheep. J. Biol. Chem. 278, 3713-3719. doi:10.1074/jbc.M210598200 
Liao, W.X., Moore, R.K., Shimasaki, S., 2004. Functional and molecular characterization of naturally occurring mutations in the oocyte-secreted factors bone morphogenetic protein-15 and growth and differentiation factor-9. J. Biol. Chem. 279, 1739117396. doi:10.1074/jbc.M401050200

Lin, J.Y., Pitman-Crawford, J.L., Bibby, A.H., Hudson, N.L., McIntosh, C.J., Juengel, J.L., McNatty, K.P., 2012. Effects of species differences on oocyte regulation of granulosa cell function. Reproduction 144, 557-567. doi:10.1530/REP-12-0267

Lin, S.J., Lerch, T.F., Cook, R.W., Jardetzky, T.S., Woodruff, T.K., 2006. The structural basis of TGF- $\beta$, bone morphogenetic protein, and activin ligand binding. Reproduction 132, 179-190. doi:10.1530/rep.1.01072

Lin, Z.-L., Li, Y.-H., Xu, Y.-N., Wang, Q.-L., Namgoong, S., Cui, X.-S., Kim, N.-H., 2014. Effects of Growth Differentiation Factor 9 and Bone Morphogenetic Protein 15 on the in vitro Maturation of Porcine Oocytes. Reprod Dom Anim 49, 219-227. doi:10.1111/rda.12254

Lipman, N.S., Jackson, L.R., Trudel, L.J., Weis-Garcia, F., 2005. Monoclonal Versus Polyclonal Antibodies: Distinguishing Characteristics, Applications, and Information Resources. ILAR J 46, 258-268. doi:10.1093/ilar.46.3.258

Li, R., Norman, R.J., Armstrong, D.T., Gilchrist, R.B., 2000. Oocyte-secreted factor(s) determine functional differences between bovine mural granulosa cells and cumulus cells. Biol. Reprod. 63, 839-845.

Li, R., Shen, Y., 2013. An old method facing a new challenge: re-visiting housekeeping proteins as internal reference control for neuroscience research. Life Sci 92, 747751. doi:10.1016/j.Ifs.2013.02.014

Liu, Y.D., Goetze, A.M., Bass, R.B., Flynn, G.C., 2011. N-terminal Glutamate to Pyroglutamate Conversion in Vivo for Human IgG2 Antibodies. J. Biol. Chem. 286, 11211-11217. doi:10.1074/jbc.M110.185041 
Macias, M.J., Martin-Malpartida, P., Massagué, J., 2015. Structural determinants of Smad function in TGF- $\beta$ signaling. Trends in Biochemical Sciences 40, 296-308. doi:10.1016/j.tibs.2015.03.012

Massagué, J., 2012. TGF $\beta$ signalling in context. Nat Rev Mol Cell Biol 13, 616-630. doi:10.1038/nrm3434

Massagué, J., Seoane, J., Wotton, D., 2005. Smad transcription factors. Genes Dev. 19, 2783-2810. doi:10.1101/gad.1350705

Mazerbourg, S., Klein, C., Roh, J., Kaivo-Oja, N., Mottershead, D.G., Korchynskyi, O., Ritvos, O., Hsueh, A.J.W., 2004. Growth differentiation factor-9 signaling is mediated by the type I receptor, activin receptor-like kinase 5. Mol. Endocrinol. 18, 653-665. doi:10.1210/me.2003-0393

McCartney-Francis, N.L., Wahl, S.M., 1994. Transforming growth factor beta: a matter of life and death. J. Leukoc. Biol. 55, 401-409.

McIntosh, C.J., Lawrence, S., Smith, P., Juengel, J.L., McNatty, K.P., 2012. Active immunization against the proregions of GDF9 or BMP15 alters ovulation rate and litter size in mice. Reproduction 143, 195-201. doi:10.1530/REP-11-0336

McIntosh, C.J., Lun, S., Lawrence, S., Western, A.H., McNatty, K.P., Juengel, J.L., 2008. The Proregion of Mouse BMP15 Regulates the Cooperative Interactions of BMP15 and GDF9. Biol Reprod 79, 889-896. doi:10.1095/biolreprod.108.068163

McMahon, G.A., Dignam, J.D., Gentry, L.E., 1996. Structural characterization of the latent complex between transforming growth factor beta 1 and beta 1-latency-associated peptide. Biochem J 313, 343-351.

McMahon, H.E., 2007. Structural and biological studies of bone morphogenetic protein-15 (PhD Thesis). University of California, San Diego.

McMahon, H.E., Hashimoto, O., Mellon, P.L., Shimasaki, S., 2008a. Oocyte-specific overexpression of mouse bone morphogenetic protein-15 leads to accelerated 
folliculogenesis and an early onset of acyclicity in transgenic mice. Endocrinology 149, 2807-2815. doi:10.1210/en.2007-1550

McMahon, H.E., Sharma, S., Shimasaki, S., 2008b. Phosphorylation of bone morphogenetic protein-15 and growth and differentiation factor-9 plays a critical role in determining agonistic or antagonistic functions. Endocrinology 149, 812-817. doi:10.1210/en.2007-1439

McNatty, K.P., Hudson, N.L., Whiting, L., Reader, K.L., Lun, S., Western, A., Heath, D.A., Smith, P., Moore, L.G., Juengel, J.L., 2007. The effects of immunizing sheep with different BMP15 or GDF9 peptide sequences on ovarian follicular activity and ovulation rate. Biol. Reprod. 76, 552-560. doi:10.1095/biolreprod.106.054361

McNatty, K.P., Juengel, J.L., Reader, K.L., Lun, S., Myllymaa, S., Lawrence, S.B., Western, A., Meerasahib, M.F., Mottershead, D.G., Groome, N.P., Ritvos, O., Laitinen, M.P.E., 2005a. Bone morphogenetic protein 15 and growth differentiation factor 9 cooperate to regulate granulosa cell function in ruminants. Reproduction 129, 481487. doi:10.1530/rep.1.00517

McNatty, K.P., Juengel, J.L., Reader, K.L., Lun, S., Myllymaa, S., Lawrence, S.B., Western, A., Meerasahib, M.F., Mottershead, D.G., Groome, N.P., Ritvos, O., Laitinen, M.P.E., 2005b. Bone morphogenetic protein 15 and growth differentiation factor 9 cooperate to regulate granulosa cell function. Reproduction 129, 473-480. doi:10.1530/rep.1.0511

McNatty, K.P., Lawrence, S., Groome, N.P., Meerasahib, M.F., Hudson, N.L., Whiting, L., Heath, D.A., Juengel, J.L., 2006. Meat and Livestock Association Plenary Lecture 2005. Oocyte signalling molecules and their effects on reproduction in ruminants. Reprod. Fertil. Dev. 18, 403-412.

McNatty, K.P., Moore, L.G., Hudson, N.L., Quirke, L.D., Lawrence, S.B., Reader, K., Hanrahan, J.P., Smith, P., Groome, N.P., Laitinen, M., Ritvos, O., Juengel, J.L., 2004. The oocyte and its role in regulating ovulation rate: a new paradigm in reproductive biology. Reproduction 128, 379-386. doi:10.1530/rep.1.00280 
McPherron, A.C., Lee, S.J., 1993. GDF-3 and GDF-9: two new members of the transforming growth factor-beta superfamily containing a novel pattern of cysteines. J. Biol. Chem. 268, 3444-3449.

Mester, B., 2013. The intraovarian cellular origins of GDF9 and BMP15 in the mouse and aspects of their biological properties (PhD Thesis). Victoria University of Wellington.

Miyazono, K., Heldin, C.-H., 1989. Role for carbohydrate structures inTGF- $\beta 1$ latency. Nature 338, 158-160. doi:10.1038/338158a0

Moore, R.K., Otsuka, F., Shimasaki, S., 2003. Molecular basis of bone morphogenetic protein-15 signaling in granulosa cells. J. Biol. Chem. 278, 304-310. doi:10.1074/jbc.M207362200

Mottershead, D.G., Pulkki, M.M., Muggalla, P., Pasternack, A., Tolonen, M., Myllymaa, S., Korchynskyi, O., Nishi, Y., Yanase, T., Lun, S., Juengel, J.L., Laitinen, M., Ritvos, O., 2008. Characterization of recombinant human growth differentiation factor-9 signaling in ovarian granulosa cells. Mol. Cell. Endocrinol. 283, 58-67. doi:10.1016/j.mce.2007.11.007

Mottershead, D.G., Ritter, L.J., Gilchrist, R.B., 2012. Signalling pathways mediating specific synergistic interactions between GDF9 and BMP15. Mol. Hum. Reprod. 18, 121128. doi:10.1093/molehr/gar056

Mottershead, D.G., Sugimura, S., Al-Musawi, S.L., Li, J.-J., Richani, D., White, M.A., Martin, G.A., Trotta, A.P., Ritter, L.J., Shi, J., Mueller, T.D., Harrison, C.A., Gilchrist, R.B., 2015. Cumulin, an Oocyte-secreted Heterodimer of the Transforming Growth Factor- $\beta$ Family, Is a Potent Activator of Granulosa Cells and Improves Oocyte Quality. J. Biol. Chem. 290, 24007-24020. doi:10.1074/jbc.M115.671487

Moustakas, A., Heldin, C.-H., 2005. Non-Smad TGF-beta signals. J. Cell. Sci. 118, 3573-3584. doi:10.1242/jcs.02554 
Mueller, T.D., Nickel, J., 2012. Promiscuity and specificity in BMP receptor activation. FEBS Letters, Sorting the TGF- $\beta$ Labyrinth 586, 1846-1859. doi:10.1016/j.febslet.2012.02.043

Otsuka, F., McTavish, K., Shimasaki, S., 2011. Integral Role of GDF-9 and BMP-15 in Ovarian Function. Mol Reprod Dev 78, 9-21. doi:10.1002/mrd.21265

Otsuka, F., Shimasaki, S., 2002. A novel function of bone morphogenetic protein-15 in the pituitary: selective synthesis and secretion of FSH by gonadotropes. Endocrinology 143, 4938-4941. doi:10.1210/en.2002-220929

Otsuka, F., Yamamoto, S., Erickson, G.F., Shimasaki, S., 2001. Bone morphogenetic protein15 inhibits follicle-stimulating hormone (FSH) action by suppressing FSH receptor expression. J. Biol. Chem. 276, 11387-11392. doi:10.1074/jbc.M010043200

Otsuka, F., Yao, Z., Lee, T., Yamamoto, S., Erickson, G.F., Shimasaki, S., 2000. Bone morphogenetic protein-15. Identification of target cells and biological functions. J. Biol. Chem. 275, 39523-39528. doi:10.1074/jbc.M007428200

Paradis, F., Novak, S., Murdoch, G.K., Dyck, M.K., Dixon, W.T., Foxcroft, G.R., 2009. Temporal regulation of BMP2, BMP6, BMP15, GDF9, BMPR1A, BMPR1B, BMPR2 and TGFBR1 mRNA expression in the oocyte, granulosa and theca cells of developing preovulatory follicles in the pig. Reproduction 138, 115-129. doi:10.1530/REP-08-0538

Paulini, F., Melo, E.O., 2011. The Role of Oocyte-Secreted Factors GDF9 and BMP15 in Follicular Development and Oogenesis. Reproduction in Domestic Animals 46, 354361. doi:10.1111/j.1439-0531.2010.01739.x

Peng, J., Li, Q., Wigglesworth, K., Rangarajan, A., Kattamuri, C., Peterson, R.T., Eppig, J.J., Thompson, T.B., Matzuk, M.M., 2013a. Growth differentiation factor 9:bone morphogenetic protein 15 heterodimers are potent regulators of ovarian functions. Proc. Natl. Acad. Sci. U.S.A. 110, E776-785. doi:10.1073/pnas.1218020110 
Peng, J., Li, Q., Wigglesworth, K., Rangarajan, A., Kattamuri, C., Peterson, R.T., Eppig, J.J., Thompson, T.B., Matzuk, M.M., 2013b. Reply to Mottershead et al.: GDF9:BMP15 heterodimers are potent regulators of ovarian functions. Proc Natl Acad Sci U S A 110, E2258. doi:10.1073/pnas.1304497110

Persani, L., Rossetti, R., Di Pasquale, E., Cacciatore, C., Fabre, S., 2014. The fundamental role of bone morphogenetic protein 15 in ovarian function and its involvement in female fertility disorders. Hum. Reprod. Update 20, 869-883. doi:10.1093/humupd/dmu036

Reader, K.L., Heath, D.A., Lun, S., McIntosh, C.J., Western, A.H., Littlejohn, R.P., McNatty, K.P., Juengel, J.L., 2011. Signalling pathways involved in the cooperative effects of ovine and murine GDF9+BMP15-stimulated thymidine uptake by rat granulosa cells. Reproduction 142, 123-131. doi:10.1530/REP-10-0490

Reader, K.L., Mottershead, D.G., Martin, G.A., Gilchrist, R.B., Heath, D.A., McNatty, K.P., Juengel, J.L., 2014. Signalling pathways involved in the synergistic effects of human growth differentiation factor 9 and bone morphogenetic protein 15. Reprod. Fertil. Dev. doi:10.1071/RD14099

Rosenzweig, B.L., Imamura, T., Okadome, T., Cox, G.N., Yamashita, H., ten Dijke, P., Heldin, C.H., Miyazono, K., 1995. Cloning and characterization of a human type II receptor for bone morphogenetic proteins. Proc Natl Acad Sci U S A 92, 7632-7636.

Rossetti, R., Di Pasquale, E., Marozzi, A., Bione, S., Toniolo, D., Grammatico, P., Nelson, L.M., Beck-Peccoz, P., Persani, L., 2009. BMP15 mutations associated with primary ovarian insufficiency cause a defective production of bioactive protein. Hum. Mutat. 30, 804-810. doi:10.1002/humu.20961

Rubenstein, P.A., 1990. The functional importance of multiple actin isoforms. Bioessays 12, 309-315. doi:10.1002/bies.950120702

Ruppert, R., Hoffmann, E., Sebald, W., 1996. Human bone morphogenetic protein 2 contains a heparin-binding site which modifies its biological activity. Eur. J. Biochem. 237, 295-302. 
Saito, S., Yano, K., Sharma, S., McMahon, H.E., Shimasaki, S., 2008. Characterization of the post-translational modification of recombinant human BMP-15 mature protein. Protein Sci 17, 362-370. doi:10.1110/ps.073232608

Sano, Y., Harada, J., Tashiro, S., Gotoh-Mandeville, R., Maekawa, T., Ishii, S., 1999. ATF-2 is a common nuclear target of Smad and TAK1 pathways in transforming growth factor-beta signaling. J. Biol. Chem. 274, 8949-8957.

Saper, C.B., 2009. A Guide to the Perplexed on the Specificity of Antibodies. J Histochem Cytochem 57, 1-5. doi:10.1369/jhc.2008.952770

Sasseville, M., Ritter, L.J., Nguyen, T.M., Liu, F., Mottershead, D.G., Russell, D.L., Gilchrist, R.B., 2010. Growth differentiation factor 9 signaling requires ERK1/2 activity in mouse granulosa and cumulus cells. J. Cell. Sci. 123, 3166-3176. doi:10.1242/jcs.063834

Schneider, C.A., Rasband, W.S., Eliceiri, K.W., 2012. NIH Image to ImageJ: 25 years of image analysis. Nat Meth 9, 671-675. doi:10.1038/nmeth.2089

Seidah, N.G., Chrétien, M., 1999. Proprotein and prohormone convertases: a family of subtilases generating diverse bioactive polypeptides. Brain Res. 848, 45-62.

Sengle, G., Charbonneau, N.L., Ono, R.N., Sasaki, T., Alvarez, J., Keene, D.R., Bächinger, H.P., Sakai, L.Y., 2008. Targeting of bone morphogenetic protein growth factor complexes to fibrillin. J. Biol. Chem. 283, 13874-13888. doi:10.1074/jbc.M707820200

Shimasaki, S., Moore, R.K., Otsuka, F., Erickson, G.F., 2004a. The bone morphogenetic protein system in mammalian reproduction. Endocr. Rev. 25, 72-101. doi:10.1210/er.2003-0007

Shimasaki, S., Moore, R.K., Otsuka, F., Erickson, G.F., 2004b. The bone morphogenetic protein system in mammalian reproduction. Endocr. Rev. 25, 72-101. doi:10.1210/er.2003-0007 
Shi, M., Zhu, J., Wang, R., Chen, X., Mi, L., Walz, T., Springer, T.A., 2011. Latent TGF- $\beta$ structure and activation. Nature 474, 343-349. doi:10.1038/nature10152

Simpson, C.M., Stanton, P.G., Walton, K.L., Chan, K.L., Ritter, L.J., Gilchrist, R.B., Harrison, C.A., 2012. Activation of latent human GDF9 by a single residue change (Gly 391 $\mathrm{Arg})$ in the mature domain. Endocrinology 153, 1301-1310. doi:10.1210/en.20111632

Sudiman, J., Sutton-McDowall, M.L., Ritter, L.J., White, M.A., Mottershead, D.G., Thompson, J.G., Gilchrist, R.B., 2014. Bone morphogenetic protein 15 in the promature complex form enhances bovine oocyte developmental competence. PLoS ONE 9, e103563. doi:10.1371/journal.pone.0103563

Sun, R.Z., Lei, L., Cheng, L., Jin, Z.F., Zu, S.J., Shan, Z.Y., Wang, Z.D., Zhang, J.X., Liu, Z.H., 2010. Expression of GDF-9, BMP-15 and their receptors in mammalian ovary follicles. J. Mol. Histol. 41, 325-332. doi:10.1007/s10735-010-9294-2

Su, Y.-Q., Wu, X., O’Brien, M.J., Pendola, F.L., Denegre, J.N., Matzuk, M.M., Eppig, J.J., 2004. Synergistic roles of BMP15 and GDF9 in the development and function of the oocyte-cumulus cell complex in mice: genetic evidence for an oocyte-granulosa cell regulatory loop. Dev. Biol. 276, 64-73. doi:10.1016/j.ydbio.2004.08.020

Taylor, S.C., Posch, A., 2014. The Design of a Quantitative Western Blot Experiment. BioMed Research International 2014, e361590. doi:10.1155/2014/361590

ten Dijke, P., Korchynskyi, O., Valdimarsdottir, G., Goumans, M.-J., 2003. Controlling cell fate by bone morphogenetic protein receptors. Mol. Cell. Endocrinol. 211, 105-113.

The National Center for Biotechnology Information (NCBI), 2015. URL http://www.ncbi.nlm.nih.gov/ (accessed 10.12.16)

Wang, B., Zhou, S., Wang, J., Liu, J., Ni, F., Yan, J., Mu, Y., Cao, Y., Ma, X., 2010. Identification of novel missense mutations of GDF9 in Chinese women with polycystic ovary syndrome. Reprod. Biomed. Online 21, 344-348. doi:10.1016/j.rbmo.2010.04.013 
Wang, T.-T., Ke, Z.-H., Song, Y., Chen, L.-T., Chen, X.-J., Feng, C., Zhang, D., Zhang, R.-J., Wu, Y.-T., Zhang, Y., Sheng, J.-Z., Huang, H.-F., 2013. Identification of a mutation in GDF9 as a novel cause of diminished ovarian reserve in young women. Hum. Reprod. 28, 2473-2481. doi:10.1093/humrep/det291

Wang, Y., Nicholls, P.K., Stanton, P.G., Harrison, C.A., Sarraj, M., Gilchrist, R.B., Findlay, J.K., Farnworth, P.G., 2009. Extra-ovarian expression and activity of growth differentiation factor 9. J. Endocrinol. 202, 419-430. doi:10.1677/JOE-08-0563

Wrana, J.L., Attisano, L., Wieser, R., Ventura, F., Massagué, J., 1994. Mechanism of activation of the TGF- $\beta$ receptor. Nature 370, 341-347. doi:10.1038/370341a0

Yan, C., Wang, P., DeMayo, J., DeMayo, F.J., Elvin, J.A., Carino, C., Prasad, S.V., Skinner, S.S., Dunbar, B.S., Dube, J.L., Celeste, A.J., Matzuk, M.M., 2001. Synergistic roles of bone morphogenetic protein 15 and growth differentiation factor 9 in ovarian function. Mol. Endocrinol. 15, 854-866. doi:10.1210/mend.15.6.0662

Zakin, L., De Robertis, E.M., 2010. Extracellular regulation of BMP signaling. Curr. Biol. 20, R89-92. doi:10.1016/j.cub.2009.11.021

Zhai, B., Liu, H., Li, X., Dai, L., Gao, Y., Li, C., Zhang, L., Ding, Y., Yu, X., Zhang, J., 2013. BMP15 Prevents Cumulus Cell Apoptosis Through CCL2 and FBN1 in Porcine Ovaries. Cellular Physiology and Biochemistry 32, 264-278. doi:10.1159/000354435

Zhu, J., Lin, S.J., Zou, C., Makanji, Y., Jardetzky, T.S., Woodruff, T.K., 2012. Inhibin $\alpha$-subunit $\mathrm{N}$ terminus interacts with activin type IB receptor to disrupt activin signaling. J. Biol. Chem. 287, 8060-8070. doi:10.1074/jbc.M111.293381 


\section{Appendices}

\section{Appendix I. Materials}

\section{Table 1: Labware}

\begin{tabular}{|c|c|}
\hline & Source/Manufacturer \\
\hline Autoclave - Systec DX 65 & Systec \\
\hline Balance - 573-36NM Precision Balance & Kern \\
\hline $\begin{array}{l}\text { Balance - Mettler XS205 Dual Range Analytical } \\
\text { Balance }\end{array}$ & Mettler Toledo \\
\hline Cell Strainer $100 \mu \mathrm{m}$ & Falcon (a Corning Brand) \\
\hline Cell Strainer $40 \mu \mathrm{m}, 70 \mu \mathrm{m}$ & Fisher Brand \\
\hline Centrifuge - Centrifuge 5810R & Eppendorf \\
\hline Centrifuge - Minispin Plus & Eppendorf \\
\hline Centrifuges tubes -Centristar $15 \mathrm{~mL}, 50 \mathrm{~mL}$ & Corning Incorporated \\
\hline $\begin{array}{l}\text { Criterion Blotter with Plate Electrodes } \\
(\text { Cat } \# 1704070)\end{array}$ & Bio-Rad \\
\hline Kimwipes - Kimtech Science Brand & Kimberly-Clark \\
\hline Fridge $-20^{\circ} \mathrm{C}$ - Tefcold & Tefcold - supplied by Inter-fridge \\
\hline Fridge $4^{\circ} \mathrm{C}$ - Tefcold & Tefcold - supplied by Inter-fridge \\
\hline Fridge $-80^{\circ} \mathrm{C}$ - Sanyo Ultralow VIP Series & Global Science (VWR), Sanyo \\
\hline Fume Cupboard - Labrocare & Thermoplastic Engineering Ltd. \\
\hline Gel Blotting Paper - GB003 & Whatman Schleicher \& Schuell \\
\hline Glass Beakers $2000 \mathrm{~mL}, 500 \mathrm{~mL}, 100 \mathrm{~mL}$ & Duran \\
\hline Glass Bottles $100 \mathrm{~mL}, 500 \mathrm{~mL}, 200 \mathrm{~mL}, 100 \mathrm{~mL}$ & Duran \\
\hline Graduated Microtubes $1.5 \mathrm{~mL}, 0.65 \mathrm{~mL}$ & Scientific Specialties Inc. (SSI) \\
\hline ImageJ Software & U. S. National Institutes of Health \\
\hline Imaging System - Omega Lum G & Aplegen \\
\hline Incubator - Incusafe & Global Science (VWR) \\
\hline $\begin{array}{l}\text { Laminar Flow - NuAire NU-437-400, class II, Type } \\
\text { A2 Biosafety Cabinet }\end{array}$ & NuAire \\
\hline Magnetic Stirrer - RCT Basic & IKA \\
\hline $\begin{array}{l}\text { Membrane - Amersham Protran nitrocellulose } \\
\text { blotting membrane }\end{array}$ & GE Healthcare Life Sciences \\
\hline Microtube Cap Locks & Scientific Specialties Inc. (SSI) \\
\hline Mini-PROTEAN Tetra Cell Casting Module & Bio-Rad (Catalogue number 165-8008) \\
\hline Mini-PROTEAN II Tetra Electrophoresis System & Bio-Rad (Catalogue number 165-8000) \\
\hline Needles - Precision-glide 26G, 20G, $18 \mathrm{G}$ & $\mathrm{BD}$ \\
\hline Parafilm “M” Laboratory Film & Pechiney Plastic Packaging \\
\hline Petri Dish 100mm- Nunclon Delta Treated & Thermo Scientific \\
\hline Petri Dish 35mm & Biofil \\
\hline Petri Dish 60mm & Citotest \\
\hline $\begin{array}{l}\text { pH meter - SevenEasy, with InLab Expert Pro } \\
\text { electrode }\end{array}$ & Mettler Toledo \\
\hline Pipette P100 & Axygen \\
\hline
\end{tabular}




\begin{tabular}{|c|c|}
\hline Pipette P1000 & Gilson \\
\hline Pipette Pump (manual) & Bel-Art- SP Scienceware \\
\hline $\begin{array}{l}\text { Pipette tips (all sizes) - Eclipse pipette tip refill } \\
\text { system }\end{array}$ & Labcon \\
\hline Pipettes P10, P2, P25, P300 & Hamilton \\
\hline Pipetting Aid (Battery operated) - Easypet & Eppendorf \\
\hline $\begin{array}{l}\text { Plastic containers for labelling membrane with } \\
\text { antibody - No } 49106 \text { PP }\end{array}$ & Uniware \\
\hline $\begin{array}{l}\text { Plastic containers for stripping membranes } \\
\text { (sealable) Klip It }{ }^{\mathrm{TM}} 200 \mathrm{~mL}\end{array}$ & Sistema \\
\hline Power Supply- PowerPac HC & Biorad \\
\hline Prism Software Version 5 & Graphpad \\
\hline Rubber Gloves - micro-touch Dermaclean & Ansell \\
\hline Scissors- dissection & World Precision Instruments Inc. (WPI) \\
\hline See-saw rocker - SSL4 & Stuart \\
\hline $\begin{array}{l}\text { Serological Pipettes - Costar Stripette } 5 \mathrm{~mL}, 10 \mathrm{~mL} \text {, } \\
25 \mathrm{~mL}\end{array}$ & Corning Incorporated \\
\hline Shaker Low Speed - Orbit LS & Labnet \\
\hline $\begin{array}{l}\text { Stereo Microscope Leica MZ9S, with Leica MDG-28 } \\
\text { light base }\end{array}$ & Leica \\
\hline Syringe Filter $0.45 \mu \mathrm{m}$ - Minisart & Sartorius Stedim Biotech \\
\hline Syringes - Luer-Lok tip $1 \mathrm{~mL}, 3 \mathrm{~mL}, 5 \mathrm{~mL}, 10 \mathrm{~mL}$ & $\mathrm{BD}$ \\
\hline Transparency Film & $3 M$ \\
\hline Tweezers - dissection & World Precision Instruments Inc. (WPI) \\
\hline $\begin{array}{l}\text { Vacuum Driven Sterile Filter - Millipore Express } \\
\text { Plus Steritop }\end{array}$ & Millipore \\
\hline Vibrating Stirrer Vibrax VXR Basic & IKA \\
\hline Vortex - Wisemix VM-10 & Wisd Laboratory Instruments \\
\hline Water Bath- Lab Companion BW-1 & Lab companion- Supplied by Bio-strategy \\
\hline Water Still- High-Q 103S & High-Q Inc. \\
\hline
\end{tabular}

\section{Table 2: Reagents and Materials}

\begin{tabular}{|l|l|l|}
\hline \multicolumn{1}{|c|}{ Reagents/materials } & \multicolumn{1}{|c|}{$\begin{array}{c}\text { Storage } \\
\text { Conditions }\end{array}$} & \multicolumn{1}{c|}{ Source/Manufacturer } \\
\hline 2-mercapto-ethanol (2-ME) & Room Temp & Sigma Life Sciences \\
\hline $30 \%$ Acrylamide/Bis Solution & $4^{\circ} \mathrm{C}$ & Bio-Rad \\
\hline Ammonium persulfate (APS) & Room Temp & Sigma-Aldrich \\
\hline Bissulfosuccinimidyl suberate (BS3) & $4^{\circ} \mathrm{C}$ & Thermo Scientific \\
\hline Bromophenol blue sodium salt & Room Temp & Sigma-Aldrich \\
\hline $\begin{array}{l}\text { Complete EDTA-free Protease inhibitor cocktail } \\
\text { tablets }\end{array}$ & $4^{\circ} \mathrm{C}$ & Roche \\
\hline Dithiothreitol (DTT) - Electrophoresis Purity & $4^{\circ} \mathrm{C}$ & Bio-Rad \\
\hline
\end{tabular}




\begin{tabular}{|c|c|c|}
\hline $\begin{array}{l}\text { E. coli. produced mature GDF9 - Sheep in } 2 M \\
\text { urea } \mathrm{pH} 8\end{array}$ & $-20^{\circ} \mathrm{C}$ & In-House \\
\hline $\begin{array}{l}\text { E. coli. produced mature BMP15 - Sheep in } 2 \mathrm{M} \\
\text { urea } \mathrm{pH} 8\end{array}$ & $-20^{\circ} \mathrm{C}$ & In-House \\
\hline Glycerol - 99\% for molecular biology & Room Temp & Sigma-Aldrich \\
\hline Glycine - Reagent Grade & Room Temp & Scharlau \\
\hline $\mathrm{HCl} 36 \%$ & Room Temp & APS Chemicals \\
\hline $\begin{array}{l}\text { HEK-293 BMP15 Recombinant Conditioned } \\
\text { Media - Deer }\end{array}$ & $\begin{array}{l}-80^{\circ} \mathrm{C} \text { long } \\
\text { term, }-20^{\circ} \mathrm{C} \\
\text { short term }\end{array}$ & In-House \\
\hline $\begin{array}{l}\text { HEK-293 BMP15 Recombinant Conditioned } \\
\text { Media - Pig }\end{array}$ & $\begin{array}{l}-80^{\circ} \mathrm{C} \text { long } \\
\text { term, }-20^{\circ} \mathrm{C} \\
\text { short term }\end{array}$ & In-House \\
\hline $\begin{array}{l}\text { HEK-293 BMP15 Recombinant Conditioned } \\
\text { Media - Sheep }\end{array}$ & $\begin{array}{l}-80^{\circ} \mathrm{C} \text { long } \\
\text { term, }-20^{\circ} \mathrm{C} \\
\text { short term }\end{array}$ & In-House \\
\hline HEK-293 GDF9 Conditioned Media - Pig & $\begin{array}{l}-80^{\circ} \mathrm{C} \text { long } \\
\text { term, }-20^{\circ} \mathrm{C} \\
\text { short term }\end{array}$ & In-House \\
\hline HEK-293 Untransfected Conditioned Media & $\begin{array}{l}-80^{\circ} \mathrm{C} \text { long } \\
\text { term, }-20^{\circ} \mathrm{C} \\
\text { short term }\end{array}$ & In-House \\
\hline HEPES & Room Temp & Sigma-Aldrich \\
\hline $\begin{array}{l}\text { Ladder - Precision Plus Protein Dual Color } \\
\text { Standards CL1610374 Rev H }\end{array}$ & $-20^{\circ} \mathrm{C}$ & Bio-Rad \\
\hline $\begin{array}{l}\text { Ladder - Kaleidoscope Prestained Standards } \\
\text { CL1610324 Rev F }\end{array}$ & $-20^{\circ} \mathrm{C}$ & Bio-Rad \\
\hline $\begin{array}{l}\text { Ladder - Prestained SDS-PAGE Standards, } \\
\text { broad Range CL1610318 Rev E }\end{array}$ & $-20^{\circ} \mathrm{C}$ & Bio-Rad \\
\hline $\begin{array}{l}\text { Medium } 199500 \mathrm{~mL}-+ \text { Earle's Salts, + L- } \\
\text { Glutamine, }+2.2 \mathrm{~g} / \mathrm{L} \text { Sodium Bicarbonate }\end{array}$ & $4^{\circ} \mathrm{C}$ & Gibco (Thermo Fisher Scientific) \\
\hline $\begin{array}{l}\text { Medium 199, powder - M5017 +Earle's salts }+\mathrm{L} \\
\text { Glutamine }\end{array}$ & $4^{\circ} \mathrm{C}$ & Sigma-Aldrich \\
\hline Methanol - AnalaR Normapur & Room Temp & VWR Prolabo \\
\hline \multicolumn{3}{|l|}{$\mathrm{NaOH}$} \\
\hline PBS -1X pH 7.4 & $4^{\circ} \mathrm{C}$ & Gibco (Thermo Fisher Scientific) \\
\hline Penicillin-Streptomycin & $-5^{\circ} \mathrm{C}$ & Gibco (Thermo Fisher Scientific) \\
\hline $\begin{array}{l}\text { Peptide B - order id 15859-3, lot } \\
\# 158501003012412 Z W, \text { Peptide Sequence: } \\
\text { QAGSIASEVPGPSRC }\end{array}$ & $-20^{\circ} \mathrm{C}$ & GenScript \\
\hline Ponceau S & Room Temp & $\mathrm{BDH}$ \\
\hline Sodium azide & Room Temp & Scharlau \\
\hline Sodium Chloride $(\mathrm{NaCl})$ - Reagent grade & Room Temp & Scharlau \\
\hline Sodium dodecyl sulfate (SDS) & Room Temp & Fisher Chemicals \\
\hline TEMED & Room Temp & Bio-Rad \\
\hline Tris base - biology grade & Room Temp & Applichem \\
\hline Tween20 & Room Temp & Fisher Bioreagents \\
\hline $\begin{array}{l}\text { WesternBright ECL-Spray Western Blotting } \\
\text { Detection System }\end{array}$ & Room Temp & Advansta \\
\hline
\end{tabular}


Table 3: Recipes

\begin{tabular}{|c|c|c|}
\hline Solution & Recipe & Storage Conditions \\
\hline $0.5 \mathrm{M}$ Tris- $\mathrm{HCl} \mathrm{pH} 6.8$ & $\begin{array}{l}6.06 \mathrm{~g} \text { Tris Base dissolved in } 80 \mathrm{~mL} \\
\text { High-Q water. Adjusted to } \mathrm{pH} 6.8 \\
\text { with concentrated } \mathrm{HCl} \text { then made up } \\
\text { to } 100 \mathrm{~mL} \text { with High-Q water. }\end{array}$ & $\begin{array}{l}\text { Room temp in Duran glass bottle (up } \\
\text { to one month) }\end{array}$ \\
\hline 1.0M Tris-HCl pH 7.5 & $\begin{array}{l}30.29 \mathrm{~g} \text { Tris Base dissolved in } 200 \mathrm{~mL} \\
\text { High-Q water. Adjusted to } \mathrm{pH} 7.5 \\
\text { with concentrated } \mathrm{HCl} \text { then made up } \\
\text { to } 250 \mathrm{~mL} \text { with High-Q water. }\end{array}$ & $\begin{array}{l}\text { Room temp in Duran glass bottle (up } \\
\text { to one month) }\end{array}$ \\
\hline 1.5M Tris- $\mathrm{HCl}$ pH 8.8 & $\begin{array}{l}36.34 \mathrm{~g} \text { Tris Base dissolved in } 160 \mathrm{~mL} \\
\text { High-Q water. Adjusted to } \mathrm{pH} 8.8 \\
\text { with concentrated } \mathrm{HCl} \text { then made up } \\
\text { to } 200 \mathrm{~mL} \text { with High-Q water. }\end{array}$ & $\begin{array}{l}\text { Room temp in Duran glass bottle (up } \\
\text { to one month) }\end{array}$ \\
\hline $10 \%(w / v)$ APS & $\begin{array}{l}1 \mathrm{~g} \text { ammonium persulphate dissolved } \\
\text { in High-Q water to give } 10 \mathrm{~mL} \text { final } \\
\text { volume }\end{array}$ & $\begin{array}{l}-20^{\circ} \mathrm{C} \text { in Graduated Microtubes (up to } \\
6 \text { months) }\end{array}$ \\
\hline $10 \%$ SDS & $\begin{array}{l}20 \mathrm{~g} \text { SDS dissolved in High-Q water to } \\
\text { give } 200 \mathrm{~mL} \text { final volume }\end{array}$ & $\begin{array}{l}\text { Room temp in Duran glass bottle (up } \\
\text { to one month) }\end{array}$ \\
\hline 10x tris-glycine stock & $\begin{array}{l}30.3 \mathrm{~g} \text { Tris base } \\
144 \mathrm{~g} \text { Glycine } \\
\text { High-Q water added to give } 1 \mathrm{~L} \text { final } \\
\text { volume when fully dissolved }\end{array}$ & $\begin{array}{l}\text { Room temp in Duran glass bottle (up } \\
\text { to one month) }\end{array}$ \\
\hline $\begin{array}{l}\text { 2x Modified laemmli } \\
\text { non-reducing LB }\end{array}$ & 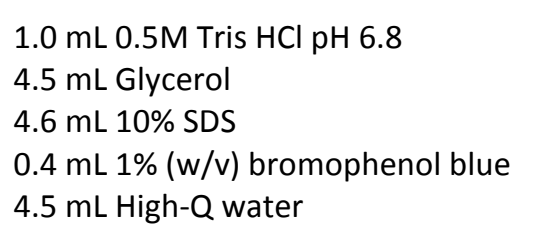 & $\begin{array}{l}-20^{\circ} \mathrm{C} \text { in Graduated Microtube aliquots } \\
\text { (up to } 3 \text { months) }\end{array}$ \\
\hline $\begin{array}{l}\text { 2x Modified laemmli } \\
\text { Reducing loading buffer }\end{array}$ & $\begin{array}{l}\text { 1.0 mL 0.5M Tris HCl pH } 6.8 \\
\text { 4.5 mL Glycerol } \\
\text { 4.6 mL 10\% SDS } \\
0.4 \mathrm{~mL} \mathrm{1 \%} \mathrm{(w/v)} \mathrm{bromophenol} \mathrm{blue} \\
\text { 4.5 mL High-Q water } \\
80 \mathrm{mg} \mathrm{DTT} \\
\text { 1.66 mL 2ME (added fresh) }\end{array}$ & $\begin{array}{l}-20^{\circ} \mathrm{C} \text { in Graduated Microtube aliquots } \\
\text { without } 2 \mathrm{ME} \text { (up to } 3 \text { months). } \\
2 \mathrm{ME} \text { stored at room temp and added } \\
\text { fresh ( } 10 \% \text { of total volume) } \\
\text { immediately before use }\end{array}$ \\
\hline
\end{tabular}




\begin{tabular}{|c|c|c|}
\hline $\begin{array}{l}\mathrm{BS}^{3} \text { Crosslinking solution } \\
(7 \mathrm{X})\end{array}$ & $\begin{array}{l}0.8 \mathrm{mg} \mathrm{BS}^{3} \\
\text { Dissolved in High-Q water to give } \\
100 \mu \mathrm{L} \text { final volume (gives } 14 \mathrm{mM} 7 \mathrm{X} \\
\text { stock solution) }\end{array}$ & Prepared fresh immediately before use \\
\hline $\begin{array}{l}\text { M199 Media for } \\
\text { collection }\end{array}$ & $\begin{array}{l}\text { 9.5g (1 jar) M199 m5017 media } \\
\text { powder } \\
10 \mathrm{~mL} \text { Pen/Strep } \\
4.766 \mathrm{~g} \text { HEPES } \\
800 \mathrm{~mL} \text { High-Q water } \\
\text { Adjusted to } \mathrm{pH} 7.3 \text { with } 2 \mathrm{M} \mathrm{NaOH} \\
\text { More High-Q water added to give } 1 \mathrm{~L} \\
\text { final volume then filtered through } \\
\text { Millipore Express Plus Steritop sterile } \\
\text { filter }\end{array}$ & $\begin{array}{l}4^{\circ} \mathrm{C} \text { in Duran glass bottle sealed with } \\
\text { Parafilm (up to } 1 \text { month) } \\
\text { Only opened in Laminar Flow and } \\
\text { aliquoted into } 50 \mathrm{~mL} \text { centrifuge tubes } \\
\text { prior to use }\end{array}$ \\
\hline $\begin{array}{l}\text { M199 Media for } \\
\text { incubation }\end{array}$ & $\begin{array}{l}\text { Medium } 199500 \mathrm{~mL} \text { (+ Earle's Salts, } \\
+ \text { L-Glutamine, + 2.2g/L Sodium } \\
\text { Bicarbonate) } \\
5 \mathrm{~mL} \text { Pen Strep }\end{array}$ & $\begin{array}{l}4^{\circ} \mathrm{C} \text { in Gibco } 500 \mathrm{~mL} \text { media bottle } \\
\text { sealed with parafilm (up to } 1 \text { month) } \\
\text { Only opened in Laminar Flow and } \\
\text { aliquoted into a } 15 \mathrm{~mL} \text { centrifuge tube } \\
\text { prior to use }\end{array}$ \\
\hline Membrane wash buffer & $\begin{array}{l}20 \mathrm{~mL} 1 \mathrm{M} \text { Tris-HCL pH } 7.5 \\
8.76 \mathrm{~g} \mathrm{NaCl} \\
1 \mathrm{~mL} \text { Tween } 20 \\
\text { High-Q water to give } 1 \mathrm{~L} \text { final volume }\end{array}$ & $\begin{array}{l}\text { Room temp in Duran glass bottle (up } \\
\text { to one month) }\end{array}$ \\
\hline Ponceau S stain & $\begin{array}{l}\text { 1.0g PonceauS } \\
2.0 \mathrm{~mL} \text { Acetic acid } \\
\text { High-Q water to give } 200 \mathrm{~mL} \text { final } \\
\text { volume }\end{array}$ & $\begin{array}{l}\text { Room temp in Duran glass bottle (up } \\
\text { to } 6 \text { months) }\end{array}$ \\
\hline $\begin{array}{l}\text { Protease inhibitor } \\
\text { solution (10X stock) }\end{array}$ & $\begin{array}{l}\text { 1X Complete EDTA-free Protease } \\
\text { inhibitor cocktail tablet } \\
5 \mathrm{~mL} \text { High-Q water }\end{array}$ & $\begin{array}{l}\text { Stored in aliquots at } \\
-20^{\circ} \mathrm{C} \text { (up to } 6 \text { months) } \\
\text { Aliquots thawed prior to use and } \\
\text { thawed aliquots were kept up to } 2 \\
\text { weeks at } 4^{\circ} \mathrm{C} \text { before disposal }\end{array}$ \\
\hline Saline solution $(0.9 \%)$ & $\begin{array}{l}9.0 \mathrm{~g} \mathrm{NaCl} \\
\text { Add High-Q water to give } 1 \mathrm{~L} \text { final } \\
\text { volume } \\
\text { Autoclaved }\end{array}$ & $\begin{array}{l}\text { Room temp in Duran glass bottle (up } \\
\text { to one month) }\end{array}$ \\
\hline Stripping buffer & $\begin{array}{l}20 \mathrm{~mL} 10 \% \text { SDS } \\
12.5 \mathrm{~mL} 0.5 \mathrm{M} \text { Tris- } \mathrm{HCl} \mathrm{pH} 6.8 \\
0.7 \mathrm{~mL} 2 \mathrm{ME} \\
\text { Add } 66.8 \mathrm{~mL} \text { High-Q water to give } \\
100 \mathrm{~mL} \text { final volume }\end{array}$ & Made fresh before use \\
\hline Transfer buffer & $\begin{array}{l}200 \mathrm{~mL} 10 X \text { Tris-Glycine stock } \\
400 \mathrm{~mL} \text { Methanol } \\
1400 \mathrm{~mL} \text { High-Q water }\end{array}$ & $\begin{array}{l}4^{\circ} \mathrm{C} \text { in Duran glass bottle up to } 2 \\
\text { months }\end{array}$ \\
\hline
\end{tabular}


Table 4: Antibodies Summary

\begin{tabular}{|c|c|c|c|c|}
\hline Antibody & $\begin{array}{l}\text { Lot } \\
\text { Number }\end{array}$ & Target Sequence & $\begin{array}{l}\text { Storage } \\
\text { conditions }\end{array}$ & Source \\
\hline $\begin{array}{l}\text { Anti } \beta \text {-Actin } \\
\text { Monoclonal Anti } \\
\beta \text {-Actin Ab produced in } \\
\text { mouse (IgG1) } \\
\text { (Product \# A5441) }\end{array}$ & 030M4788 & $\begin{array}{l}\text { DDDIAALVIDNGSGK } \\
\text { conjugated to KLH }\end{array}$ & $\begin{array}{l}-20^{\circ} \mathrm{C} \text { in liquid } \\
\text { of processed } \\
\text { mouse ascites } \\
\text { fluid and } \\
15 \mathrm{mM} \text { sodium } \\
\text { azide } \\
\end{array}$ & Sigma-Aldrich \\
\hline $\begin{array}{l}\text { HRP-conjugated antibody } \\
\text { Peroxidase-conjugated } \\
\text { Affinipure Rabbit Anti- } \\
\text { mouse IgG }(\mathrm{H}+\mathrm{L} \text { ) } \\
\text { (peroxidase purified from } \\
\text { horseradish root) }\end{array}$ & 119936 & $\begin{array}{l}\text { Reacts with whole } \\
\text { molecule mouse IgG and } \\
\text { the light chains of other } \\
\text { mouse immunoglobulins }\end{array}$ & $\begin{array}{l}-20^{\circ} \mathrm{C} \text { stored } \\
\text { at } 0.4 \mathrm{mg} / \mathrm{mL} \\
\text { in equal parts } \\
\text { High-Q water } \\
\text { and glycerol }\end{array}$ & $\begin{array}{l}\text { Jackson } \\
\text { ImmunoResearch } \\
\text { Laboratories Inc. }\end{array}$ \\
\hline $\begin{array}{l}\text { Mab61A } \\
\text { Anti BMP15 (Targeted at } \\
\text { N-terminal flexible region } \\
\text { of mature peptide in } \\
\text { sheep) }\end{array}$ & N/A & $\begin{array}{l}\text { SEVPGPSREHDGPES } \\
\text { sequence towards } \mathrm{N} \text { - } \\
\text { terminal of mature ovine } \\
\text { BMP15. }\end{array}$ & $\begin{array}{l}4^{\circ} \mathrm{C} \text { in } \\
\mathrm{PBS} / 0.1 \% \\
\text { sodium azide }\end{array}$ & $\begin{array}{l}\text { Oxford Brookes } \\
\text { University in the } \\
\text { United Kingdom } \\
\text { under contract to } \\
\text { AgResearch }\end{array}$ \\
\hline $\begin{array}{l}\text { Mab61B } \\
\text { Anti BMP15 (Targeted at } \\
\text { N-terminal flexible region } \\
\text { of mature peptide in } \\
\text { sheep) }\end{array}$ & N/A & $\begin{array}{l}\text { QAGSIASEVPGPSR(C) } \\
\text { sequence at N-terminal } \\
\text { of mature ovine BMP15. } \\
\text { Cysteine residue } \\
\text { (underlined) used to } \\
\text { chemically conjugate } \\
\text { sequence to KLH }\end{array}$ & $\begin{array}{l}4^{\circ} \mathrm{C} \text { in } \\
\mathrm{PBS} / 0.1 \% \\
\text { sodium azide }\end{array}$ & $\begin{array}{l}\text { by GenScript }{ }^{\circledast}, \text { USA } \\
\text { under contract to } \\
\text { Victoria University } \\
\text { of Wellington }\end{array}$ \\
\hline $\begin{array}{l}\text { Mab37A } \\
\text { Anti GDF9 (Targeted at N- } \\
\text { terminal flexible region of } \\
\text { mature peptide in sheep) }\end{array}$ & N/A & $\begin{array}{l}\text { DQESASSELKKPLV( } \underline{\mathrm{C}}) \\
\text { sequence at N-terminal } \\
\text { of mature ovine BMP15. } \\
\text { Cysteine residue } \\
\text { (underlined) used to } \\
\text { chemically conjugate } \\
\text { sequence to KLH }\end{array}$ & $\begin{array}{l}4^{\circ} \mathrm{C} \text { in } \\
\mathrm{PBS} / 0.1 \% \\
\text { sodium azide }\end{array}$ & $\begin{array}{l}\text { Oxford Brookes } \\
\text { University in the } \\
\text { United Kingdom } \\
\text { under contract to } \\
\text { AgResearch }\end{array}$ \\
\hline
\end{tabular}




\section{Appendix II. Antibodies}

\section{Mab61A- Anti-BMP15}

Mab61A was a mouse generated monoclonal antibody (IgG) created to target BMP15 and was made by Oxford Brookes University in the United Kingdom under contract to AgResearch according to the protocol in Juengel et al., 2002. The methods described outbred Tyler's original mice being immunized with full length $E$. coli. produced mature ovine BMP15 protein in Freund complete adjuvant. Positive clones were screened by ELISA using HEK-293 produced ovine BMP15 conditioned media and the best clone was selected (McNatty et al., 2005a). The supernatant of the best clone, which contained Mab61A, was then purified using protein $\mathrm{G}$ affinity purification. It was later determined that the Mab61A antibody targets a 15 amino acid sequence towards the $\mathrm{N}$-terminus of the mature peptide, SEVPGPSREHDGPES (see Appendix II Figure 1) (McNatty et al., 2005a). The region where the target sequence (SEVPGPSREHDGPES) is located is predicted to be a flexible region with unknown function based on a BMP15 dimer model previously described (Juengel \& McNatty, 2005; McNatty et al., 2007). In one study, the same 15 amino acid peptide was conjugated to Keyhole limpet hemocyanin $(\mathrm{KLH})$ via a C-terminal cysteine residue, and then used in in-vivo immunization experiments in sheep where it was shown to elicit a strong immune response which in turn inhibited ovarian function (McNatty et al., 2007). This was evidenced by a significant decline in the number of corpus luteum following immunization with the peptide, as well as an increase in abnormal follicle morphology (McNatty et al., 2007). Mab61A has been used to successfully immuno-neutralise and inhibit the bioactivity of ovine BMP15 in vitro (Lin et al., 2012).

\section{Mab61B- Anti-BMP15}

Mab61B was also a mouse generated monoclonal antibody (isotype $\lg G 1, \mathrm{k}$ ) which was created to target BMP15 and was made by GenScript ${ }^{\circledR}$, USA under contract to Victoria University of Wellington. It was generated in mice using a synthesized 14 amino acid sequence from the $\mathrm{N}$-terminal of the mature ovine BMP15 sequence which was conjugated 
to KLH via a cysteine residue (QAGSIASEVPGPSR(ㅡ)-KLH) (see Appendix II Figure 1). Positive clones in supernatant samples were screened by ELISA using the same 15 amino acid sequence (QAGSIASEVPGPSRC) without the KLH attached and the best clone was selected. The supernatant of the best clone which contained Mab61B was then purified using protein A/G affinity purification. The Mab61B target sequence shared similar properties to the Mab61A target sequence due to their close proximity. It is predicted to be in a flexible region of the mature peptide in a region with unknown function based on a BMP15 dimer model (Juengel \& McNatty, 2005; McNatty et al., 2007). An in-vivo study that used the same peptide-KLH immunogen which was used to generate Mab61B (QAGSIASEVPGPSR(C)-KLH) was used to successfully immunize sheep, and the associated immune response was shown to strongly inhibit ovarian function (McNatty et al., 2007). The effect of immunizing with the Mab61B target sequence was even more disruptive to ovarian function than the effect of immunization with the Mab61A target sequence as evidenced by a marked decline in the number of corpus luteum over a shorter time period following immunization (Kenneth P. McNatty et al., 2007). Eight amino acids in the Mab61B target sequence overlap with the Mab61A target sequence (see Appendix II Figure 1).

\section{Mab37A- Anti-GDF9}

Mab37A is a mouse generated monoclonal antibody $(\operatorname{IgG})$ which was made by Oxford Brookes University in the United Kingdom under contract to AgResearch. The antibody was generated using full length $E$. coli. produced mature ovine GDF9 protein along with Freund complete adjuvant. Mab37A has been shown previously to specifically bind ovine GDF9, and to be able to detect the promature and mature forms of the protein (Lin et al., 2012; McIntosh et al., 2008; McNatty et al., 2005a). It has not previously been established whether the antibody can inhibit GDF9 bioactivity in vitro. During the antibody production and characterisation process it was determined that there was some cross reactivity with ovine BMP15 (data not published). The specific target region of Mab37A on the mature ovine GDF9 molecule has not been established. 


\section{Homology \%}

RAT SDNPCPSQEQRDRSN 40.0\%

PIG SEVIGGSREHDGPES 93.3\%

SHEEP SEVPGPSREHDGPES

DEER SEVPGPSREHDGPES

*

\section{B - Mab61B}

\section{RAT OTISIASDVPCPSO 64.3\% \\ PIG QAGSIASEVDGPSR \\ SHEEP QAGSIASEVPGPSR \\ DEER QAGSIASEVPGPSR

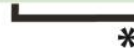

\footnotetext{
* Region of overlap between both antibody targets
}

\section{Residue Properties:}

RED- Small, hydrophobic, Aromatic (except Y)

BLUE - Acidic

PURPLE - Basic (except H)

GREEN- Hydroxyl + Sulfhydryl + amine $+G$

Appendix II Figure 1. The mature BMP15 target sequences for Mab61A and Mab61B. Black boxes around individual amino acids indicate differences from the original sheep target sequence. The colours of the residues indicate the properties as shown by the key in the figure. The level of homology shared with the original sheep target sequence is indicated next to each sequence. (A) The 15 amino acid target sequence for Mab61A in mature sheep BMP15 is displayed alongside the equivalent region of mature BMP15 in rats, pigs and deer. The target sequence is located 6 amino acids in from the N-terminal of the mature BMP15 peptide. (B) The 15 amino acid target sequence for Mab61B in mature sheep BMP15 is displayed alongside the equivalent region of mature BMP15 in rats, pigs and deer. The target sequence is located directly at the $\mathrm{N}$-terminal of the mature BMP15 peptide. The asterisk $\left({ }^{*}\right)$ and bracket indicate the 8 amino acid region where the targets of each antibody overlap.

\section{Appendix III. Recombinant BMP15 and GDF9 Samples}

The recombinant BMP15 conditioned media samples were generated in-house at Victoria University Wellington.

The HEK-293 samples were generated by transferring the genes for the required protein into the pEFIRES-P expression vector (Hobbs, Jitrapakdee, \& Wallace, 1998), and then transfected into a human embryonic kidney cell line (HEK-293). High recombinant protein expressing clones were selected and the protein samples were collected in serum-free harvesting medium (DMEM/Ham's F-12, 1:1) with I-glutamine, penicillin, streptomycin, 0.01\% BSA and heparin added as described in McNatty et al. (2005a). 
Escherichia coli (E. coli) produced recombinant protein samples were generated by transferring the cDNA mature BMP15 sequence into the $\mathrm{pET} 23 \mathrm{~b}$ expression vector. The vector was then transformed into BL21DE3 E. coli cells which were then used for protein production as described in Juengel et al. (2002).

\section{Appendix IV. Example of full blot from Figure 3.11}

A Sheep BMP15- Reducing

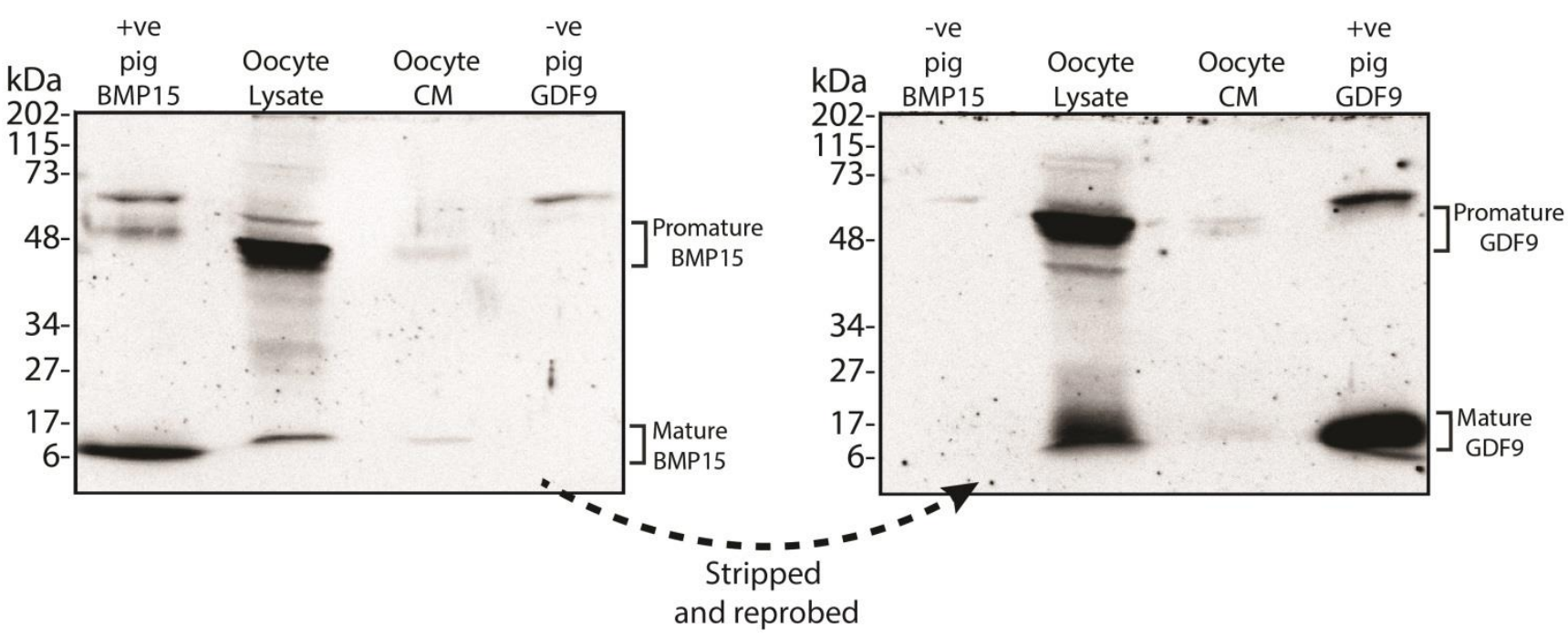

Appendix IV Figure 1. Full blots representing sheep BMP15 and GDF9 under reducing conditions. (A) A sample of a full Western blot from Figure 3.11 representing BMP15 in sheep oocyte samples under reducing conditions. The blot was labelled with the anti-BMP15 antibody Mab61A. Lane 1 contains $2 \mu \mathrm{L}$ of recombinant HEK-293 produced pig BMP15 conditioned media (positive control), Lane 2 contains the lysate from 100 sheep oocytes, Lane 3 contains the conditioned media from 125 sheep oocytes, Lane 4 contains $2 \mu \mathrm{L}$ of recombinant HEK-293 produced pig GDF9 conditioned media (negative control). (B) Represents the same blot from (A) after it has been stripped twice and labelled with the anti-GDF9 antibody Mab37A. Lane 4 now represents the positive control and Lane 1 represents the negative control. The positions of the promature and mature forms of each protein are indicated on the side of each blot. 